\title{
The state-of-the-art of preconditioners for sparse linear least-squares problems
}

Article

Accepted Version

Scott, J. and Gould, N. (2017) The state-of-the-art of preconditioners for sparse linear least-squares problems. ACM Transactions on Mathematical Software (TOMS), 43 (4). 36. ISSN 0098-3500 doi: https://doi.org/10.1145/3014057 Available at https://centaur.reading.ac.uk/70342/

It is advisable to refer to the publisher's version if you intend to cite from the work. See Guidance on citing.

To link to this article DOI: http://dx.doi.org/10.1145/3014057

Publisher: ACM

All outputs in CentAUR are protected by Intellectual Property Rights law, including copyright law. Copyright and IPR is retained by the creators or other copyright holders. Terms and conditions for use of this material are defined in the End User Agreement.

\section{www.reading.ac.uk/centaur}

\section{CentAUR}

Central Archive at the University of Reading

Reading's research outputs online 


\title{
The state-of-the-art of preconditioners for sparse linear least-squares problems
}

\author{
NICHOLAS GOULD and JENNIFER SCOTT, Rutherford Appleton Laboratory
}

\begin{abstract}
In recent years, a variety of preconditioners have been proposed for use in solving large sparse linear leastsquares problems. These include simple diagonal preconditioning, preconditioners based on incomplete factorizations and stationary inner iterations used with Krylov subspace methods. In this study, we briefly review preconditioners for which software has been made available and then present a numerical evaluation of them using performance profiles and a large set of problems arising from practical applications. Comparisons are made with state-of-the-art sparse direct methods.
\end{abstract}

Categories and Subject Descriptors: G.1.3 [Numerical Linear Algebra]: Linear systems (direct and iterative methods); G.1.6 [Optimization]: Least squares methods

General Terms: Algorithms, Performance

Additional Key Words and Phrases: Least-squares problems, normal equations, augmented system, sparse matrices, direct solvers, iterative solvers, preconditioning

ACM Reference Format:

Nicholas Gould and Jennifer Scott, 2016. The state-of-the-art of preconditioners for sparse linear leastsquares problems. ACM Trans. Math. Softw. V, N, Article XXXX (XXXX 2015), 41 pages.

DOI : http://dx.doi.org/10.1145/0000000.0000000

\section{INTRODUCTION}

The method of least-squares is a commonly used approach to find an approximate solution of overdetermined or inexactly specified systems of equations. Since the development of the principle of least squares by Gauss in 1795 [23], the solution of least-squares problems has been, and continues to be, a fundamental method in scientific data fitting. Least-squares solvers are used across a wide range of disciplines, for everything from simple curve fitting, through the estimation of satellite image sensor characteristics, data assimilation for weather forecasting and for climate modelling, to powering internet mapping services, exploration seismology, NMR spectroscopy, piezoelectric crystal identification (used in ultrasound for medical imaging), aerospace systems, and neural networks.

In this study, we are interested in the important special case of the linear leastsquares problem,

$$
\min _{x}\|b-A x\|_{2}
$$

This work was supported by EPSRC grants EP/I013067/1 and EP/M025179/1.

Authors' addresses: N. I. M. Gould and J. A. Scott, Scientific Computing Department, STFC Rutherford Appleton Laboratory, Harwell Campus, Oxfordshire, OX11 0QX, UK, nick.gould@stfc.ac.uk, jennifer.scott@stfc.ac.uk

Permission to make digital or hard copies of all or part of this work for personal or classroom use is granted without fee provided that copies are not made or distributed for profit or commercial advantage and that copies bear this notice and the full citation on the first page. Copyrights for components of this work owned by others than ACM must be honored. Abstracting with credit is permitted. To copy otherwise, or republish, to post on servers or to redistribute to lists, requires prior specific permission and/or a fee. Request permissions from permissions@acm.org.

(c) YYYY ACM. 0098-3500/YYYY/-ARTA $\$ 15.00$

DOI : http://dx.doi.org/10.1145/0000000.0000000 
where $A \in \mathbb{R}^{m \times n}$ with $m \geq n$ is large and sparse and $b \in \mathbb{R}^{m}$. Solving (1) is mathematically equivalent to solving the $n \times n$ normal equations

$$
C x=A^{T} b, \quad C=A^{T} A,
$$

and this, in turn, is equivalent to solving the $(m+n) \times(m+n)$ augmented system

$$
K y=c, \quad K=\left[\begin{array}{cc}
I_{m} & A \\
A^{T} & 0
\end{array}\right], \quad y=\left[\begin{array}{c}
r(x) \\
x
\end{array}\right], \quad c=\left[\begin{array}{l}
b \\
0
\end{array}\right],
$$

where $r(x)=b-A x$ is the residual vector and $I_{m}$ is the $m \times m$ identity matrix. Increasingly, the sizes of the problems that scientists and engineers wish to solve are getting larger (problems in many millions of variables are becoming typical); they are also often ill-conditioned. In other applications, it is necessary to solve many thousands of problems of modest size and so efficiency in this case is essential. The normal equations are attractive in that they are always consistent and positive semidefinite (positive definite if $A$ is of full column rank). However, a well-known drawback is that the condition number of $C$ is the square of the condition number of $A$ so that the normal equations are often highly ill-conditioned [10]. Furthermore, the density of $C$ can be much greater than that of $A$ (if $A$ has a single dense row, $C$ will be dense). The main disadvantages of working with the augmented system are that $K$ is symmetric indefinite and is much larger than $C$ (particularly if $m \gg n$ ).

Two main classes of methods may be used to try and solve these linear systems: direct methods and iterative methods. A direct method proceeds by computing an explicit factorization, either a sparse $L L^{T}$ Cholesky factorization of the normal equations (2) (assuming $A$ is of full column rank so that $C$ is positive definite) or a sparse $L D L^{T}$ factorization of the augmented system (3). Alternatively, a $Q R$ factorization of $A$ may be used, that is, a "thin" $Q R$ factorization of the form

$$
A=Q\left[\begin{array}{c}
R \\
0
\end{array}\right],
$$

where $Q$ is an $m \times m$ orthogonal matrix and $R$ is an $n \times n$ upper triangular matrix. Whilst direct solvers are generally highly reliable, iterative methods may be preferred because they often require significantly less storage and in some applications it may not be necessary to solve the system with the high accuracy offered by a direct solver. However, the successful application of an iterative method often requires a suitable preconditioner to achieve acceptable (and ideally, fast) convergence rates. Currently, there is much less knowledge of preconditioners for least-squares problems than there is for sparse symmetric linear systems and, as remarked in [12], "the problem of robust and efficient iterative solution of least-squares problems is much harder than the iterative solution of systems of linear equations". This is, at least in part, because $A$ does not have the properties of differential problems that can make standard preconditioners effective.

In the past decade or so, a number of different techniques for preconditioning Krylov subspace methods for least-squares problems have been developed. A brief overview with a comprehensive list of references is included in the introduction to the recent paper by Bru et al [12]. However, in the literature the reported experiments on the performance of different preconditioners are often limited to a small set of problems, generally arising from a specific application. Moreover, they may use prototype codes that are not available for others to test and they may only be run using MATLAB. Our aim is to perform a wider study in which we use a large set of test problems to evaluate the performance of a range of preconditioners for which software has been made available. The intention is to gain a clearer understanding of when 
particular preconditioners perform well (or, indeed, perform poorly) and we will use this to influence our future work on linear least-squares. Our attention is limited to preconditioners for which software in Fortran or $\mathrm{C}$ is available; it is beyond the scope of this work to provide efficient and robust implementations for all the approaches that have appeared in the literature (although even then, as we discuss in Section 8, we have found it necessary in some cases to modify and possibly re-engineer some of the existing software to make it suitable for use in this study).

The rest of the paper is organised as follows. In Section 2, we describe our test environment, including the set of problems used in this study. Direct solvers for solving the normal equations and/or the augmented system are briefly recalled in Section 3. One of these (HSL_MA97) is used for comparison with the performance of the preconditioned iterative methods. In Section 4, we report on experiments with two methods, LSQR and LSMR, that are mathematically equivalent to applying conjugate gradients and MINRES, respectively, to the normal equations but have favourable numerical properties. On the basis of our findings, LSMR is used in the rest of our experiments. Preconditioning strategies are briefly described in Sections 5 to 7 . The software used in our experiments is discussed in Section 8. We present numerical results in Section 10 and finally, in Section 11, concluding remarks are made.

\section{TEST ENVIRONMENT}

\begin{tabular}{|c|c|}
\hline & $\begin{array}{l}\text { Table I. } \\
\text { Test } \\
\text { Machine } \\
\text { Characteristics. }\end{array}$ \\
\hline Processor & $8 \times$ Intel i7-4790 $(3.6 \mathrm{GHz})$ \\
\hline Memory & 15.6 Gbytes \\
\hline Compiler & gfortran version 4.7 with option - \\
\hline BLAS & open BLAS (serial) or Intel MKL (serial vs parallel) \\
\hline
\end{tabular}

The characteristics of the machine used to perform our tests are given in Table I. In our experiments, the direct solvers (see Section 3) are run in parallel, using four processors. Our initial experiments on iterative methods (those in Sections 4 and 8) are run on a single processor, although where BLAS are used, these may take advantage of parallelism. Later, when comparing iterative and direct approaches (in Sections 9 and 10), we repeat the calculations on 4 processors for the methods we have found to be best. Here sparse matrix-vector products and sparse triangular solves (if any) required by the preconditioner are performed in parallel using Intel Mathematics Kernel Library (MKL) routines; no attempt is made to parallelize any of the iterative methods themselves, nor the software for constructing the preconditioners.

Throughout this study, all reported times are elapsed times in seconds measured using the Fortran system_clock. For each solver and each problem, a time limit of 600 seconds is imposed; if this limit is exceeded, the solver is flagged as having failed on that problem. Failures resulting from insufficient memory are also flagged and, in the case of the iterative solvers, the number of iterations per problem is limited to $10^{7}$. We observe that, although the tests were performed on a lightly loaded machine, the timings can vary if the experiments are repeated. In our experience, this variation is small (typically less than 5\%), although for large problems for which memory becomes an issue, the variation can be more significant. Unfortunately, given the large scale nature of this study and time taken to perform the experiments, it was not possible to produce average timings. However, variations in time that may arise from reruns will have little effect on the conclusions we can draw from the performance profiles that 
we use as our main tool for assessing performance (see Section 2.3). In order to obtain close-to-repeatable times when running in parallel on 4 processors, we specify which processors are to be used via the Linux numactl $-C$ 0,1,2,3 command.

\subsection{Test problems}

The problems used in our study are all taken from the CUTEst linear programme set [26] and the UFL Collection [18]. To determine the test set that we shall use for the majority of our experiments, we selected all the rectangular matrices $A$ and removed "duplicates" (that is, similar problems belonging to same group), leaving a single representative. This gave us a set of 921 problems. In all our tests, we check $A$ for duplicate entries (they are summed), out-of-range entries (they are removed) and explicit zeros (they are removed). In addition, $A$ is checked for null rows and columns. Any such rows or columns are removed and if, after removal $n>m$, the matrix is transposed. The computation then continues with the resulting cleaned matrix. If values for the matrix entries are not supplied, we generate random values in the range $(-1,1)$.

To ensure we only include non-trivial problems, for each cleaned matrix we solved the normal equations (2) using LSMR (see Section 4, with the local reorthogonalization parameter set to 10) without preconditioning and retained those problems for which convergence (using the stopping criteria discussed in Section 2.2) was not achieved within 100,000 iterations or required more than 10 seconds (when run on a single processor). Using the provided right-hand side vector $b$ if available or taking $b$ to be the vector of 1's if not (so that the problems are not necessarily consistent but at the same time this choice makes it straightforward to regenerate the same $b$ for running tests with a range of solvers) resulted in a test set $\mathcal{T}$ of 83 problems. This set is listed along with some of the characteristics of each problem (including the number of entries, the density of the row with the most entries, an estimate of the deficiency in the rank) in Table I in the Appendix (see [30] for details of the full set).

\subsection{Stopping criteria}

Recall that the linear LS problem we seek to solve is

$$
\min \phi(x), \quad \phi(x)=\|r(x)\|_{2},
$$

where $r(x)=b-A x$ is the residual. If the minimum residual is zero, $\phi(x)$ is non differentiable at the solution and so the first check we make at iteration $k$ is on the $k$ th residual $\left\|r_{k}\right\|_{2}$, where $r_{k}=b-A x_{k}$ with $x_{k}$ the computed solution on the $k$ th iteration. If the minimum residual is non zero then

$$
\nabla \phi(x)=-\frac{A^{T} r(x)}{\|r(x)\|_{2}}
$$

and we want to terminate once $\nabla \phi(x)$ is small. Thus, in our tests with iterative solvers we use the following stopping rules:

- C1: Stop if $\left\|r_{k}\right\|_{2}<\delta_{1}$.

- C2: Stop if

$$
\frac{\left\|A^{T} r_{k}\right\|_{2}}{\left\|r_{k}\right\|_{2}}<\frac{\left\|A^{T} r_{0}\right\|_{2}}{\left\|r_{0}\right\|_{2}} * \delta_{2}
$$

where $A$ is the "cleaned" matrix and $\delta_{1}$ and $\delta_{2}$ are convergence tolerances that we set to $10^{-8}$ and $10^{-6}$, respectively. In all our experiments, we take the initial solution guess 
to be $x_{0}=0$ and in this case $\mathrm{C} 2$ reduces to

$$
\frac{\left\|A^{T} r_{k}\right\|_{2}}{\left\|r_{k}\right\|_{2}}<\frac{\left\|A^{T} b\right\|_{2}}{\|b\|_{2}} * \delta_{2}
$$

Note that these stopping criteria are independent of the preconditioner and thus they enable us to compare the performances of different preconditioners. In the case of no preconditioning, these stopping criteria are closely related to those used by Fong and Saunders [24] in their 2010 implementation of LSMR (see http: //web.stanford.edu/group/SOL/download.html). However, if a preconditioner is used, the Fong and Saunders implementation bases the stopping criteria on $\left\|\left(A M^{-1}\right)^{T} r\right\|_{2}$, where $M$ is the (right) preconditioner. This means that a different test is applied for different preconditioners and thus is not appropriate for comparing the performances of different preconditioners. Using $\mathrm{C} 1$ and $\mathrm{C} 2$ involves additional work; in our tests, we have chosen to exclude the cost of computing the residuals for testing $\mathrm{C} 1$ and $\mathrm{C} 2$ from the reported runtimes (and from the 600s time limit per problem) and we use a modified reverse communication version of LSMR that enables us to use C1 and C2 in place of the Fong and Saunders stopping criteria. We note that new results on estimating backward errors for least-squares problems have been derived by a number of authors, including [31; 42].

\subsection{Performance profiles}

To assess the performance of different solvers on our test set $\mathcal{T}$, we report the raw data but we also employ performance profiles [19], which in recent years have become a popular and widely used tool for providing objective information when benchmarking software. The performance ratio for an algorithm on a particular problem is the performance measure for that algorithm divided by the smallest performance measure for the same problem over all the algorithms being tested (here we are assuming that the performance measure is one for which smaller is better, for example, the iteration count or time taken). The performance profile is the set of functions $\left\{p_{i}(f): f \in[1, \infty)\right\}$, where $p_{i}(f)$ is the proportion of problems where the performance ratio of the $i$ th algorithm is at most $f$. Thus $p_{i}(f)$ is a monotonically increasing function taking values in the interval $[0,1]$. In particular, $p_{i}(1)$ gives the fraction of the examples for which algorithm $i$ is the winner (that is, the best according to the performance measure), while if we assume failure to solve a problem (for example, through the maximum iteration count or time limit being exceeded) is signaled by a performance measure of infinity, $p_{i}^{*}:=\lim _{f \rightarrow \infty} p_{i}(f)$ gives the fraction for which algorithm $i$ is successful. If we are just interested in the number of wins, we need only compare the values of $p_{i}(1)$ for all the algorithms but, if we are interested in algorithms with a high probability of success, we should choose the ones for which $p_{i}^{*}$ has the largest values. In our performance profile plots, we use a logarithmic scale in order to observe the performance of the algorithms over a large range of $f$ while still being able to discern in some detail what happens for small $f$.

Whilst performance profiles are a very helpful tool when working with a large test set and several algorithms, as Dolan and Moré point out, they do need to be used and interpreted with care. This is especially true if we want to try and rank the algorithms in order. Our preliminary experiments for this study led us to re-examine performance profiles [29]. We found that, while they give a clear measure of which is the better algorithm for a chosen $f$ and given set $\mathcal{T}$, if performance profiles are used to compare more than two algorithms, they determine which algorithm has the best probability $p_{i}(f)$ for $f$ in a chosen interval, but we cannot necessarily assess the performance of one algorithm relative to another that is not the best using a single performance profile 
plot. Thus in Section 10, we limit some of our performance profiles to two solvers at a time.

\subsection{Parameter setting}

Where codes offer a number of options (such as orderings and scalings), we normally use the default or otherwise recommended settings; no attempt is made to tune the parameters for a particular problem (this would not be realistic given the size of the test set and number of solvers). However, it is recognised that, for some examples, choosing settings other than the defaults may significantly enhance performance (or adversely effect it) and, in practice, a user may find it advisable to invest time in experimenting with different choices to try and optimize performance for his/her application. Details of the software we use are given in Section 8, together with the parameter settings.

\section{DIRECT SOLVERS}

While the focus of our study is on preconditioning iterative methods for least-squares problems, it is of interest to look at how these methods perform in comparison to sparse direct methods. For the normal equations (2), a positive definite solver that computes a sparse Cholesky factorization can be used, such as CHOLMOD [14] or HSL_MA87 [37]. Alternatively, there are sparse packages that can factorize both positive definite and indefinite systems. These include a number of HSL codes (notably, MA57 [20], HSL_MA86 [39], and HSL_MA97 [38]) as well as MUMPS [50], WSMP [33], PARDISO [56] and SPRAL_SSIDS [36]. Comparisons of some of these packages for solving sparse linear systems may be found in [25; 28]. When used to solve the augmented system (3), the solvers employ some kind of pivoting to try and ensure numerical stability, and this can impose a non trivial runtime overhead (as well as adding significantly to the complexity of the software and the memory requirements).

Most modern sparse direct solvers are designed to run in parallel, either through the use of MPI, OpenMP or GPUs. It is beyond the scope of the current study to conduct a detailed comparison of the performance of direct solvers on least-squares problems; instead we opt to use HSL_MA87 (Version 2.4.0) for the normal equations and HSL_MA97 (Version 2.3.0) for the augmented system in our comparisons with iterative methods. This choice was made since HSL_MA87 and HSL_MA97 are recent state-of-the-art packages that are designed for multicore machines, and, because we are responsible for their development, we find them convenient to use and to incorporate into our test environment. We note that CHOLMOD has an attractive feature that is not currently offered by any of the HSL codes which is that it can factor the normal equations without being given $C$ explicitly; just providing $A^{T}$ suffices and this saves memory.

For sparse QR, far fewer software packages have been developed. Those that are available include MA49 [2] from the mid 1990s and, much more recently, SuiteSparseQR [17] and qr_mumps [13]. Here we use SuiteSparseQR version 4.4.4 (for which we have written a Fortran interface).

Although a straightforward application of a direct method to (2) or (3) is usually successful, computer rounding can have a profound effect. In particular, for problems that are (or are close to) rank deficient, simply forming the (theoretically) positive semidefinite normal matrix $C$ may result in a matrix that is slightly indefinite and a Cholesky factorization will breakdown. Likewise, the symmetric, indefinite factorization of the augmented matrix $K$ may produce a numerical inertia (i.e., a count of the numbers of positive, negative and zero eigenvalues) that is impossible had the matrix been factorized exactly. Thus, in addition to applying the appropriate factorization routine to our test problems, we also consider employing a "scale-andshift" strategy that aims to reduce the impact of poor conditioning. In particular, we 
modify the normal equations and augmented system so that the required solution $x$ is $x=S z$, where $z$ is the solution of the system

$$
\bar{C} z=\bar{A}^{T} b, \quad \bar{C}=\bar{A}^{T} \bar{A}+\delta_{C} I_{n},
$$

or

$$
\bar{K} \bar{y}=c, \quad \bar{K}=\left[\begin{array}{cc}
I_{m} & \bar{A} \\
\bar{A}^{T} & -\delta_{K} I_{n}
\end{array}\right], \quad y=\left[\begin{array}{c}
r(x) \\
z
\end{array}\right], \quad c=\left[\begin{array}{l}
b \\
0
\end{array}\right],
$$

where $\bar{A}=A S$ and the diagonal matrix $S$ scales the columns of $A$ so that each has a unit 2-norm, and the scalars $0<\delta_{C}, \delta_{K} \ll 1$. In our experiments, we have found that $\delta_{C}=10^{-12}$ and $\delta_{K}=10^{-10}$ are generally suitable choices, and although they necessarily perturb the computed solution, our experience is that the perturbation is sufficiently small to be acceptable. Similar regularizations have been proposed by many authors, e.g., [63; 65]. On our test set $\mathcal{T}$, HSL_MA87 and HSL_MA97 both failed to solve 26 of the 83 problems without prescaling and shifting (the solvers used their own default scalings of $C$ and $K$, respectively), and this reduced to 15 and 20, respectively, with prescaling and shifting (see Tables 4.17-4.20 in [30] for details). Thus, in what follows, we use the HSL direct solvers combined with the scale-and-shift approach in all remaining comparisons.

A time performance profile comparing SuiteSparseQR (denoted by SPQR), HSL_MA87 applied to the normal equations (MA87 normal equations) and HSL_MA97 applied to the the augmented system (MA97 augmented system) is given in Figure 1. In our experiments, one step of iterative refinement was used. We see that using HSL_MA87 for the normal equations leads to the smallest number of failures while it is the fastest approach for more than half of the problems. The failures are for some of the largest problems and are because of insufficient memory (see Tables 4.17-4.21 in [30] for details). In addition, for SPQR there are three problems (f855_mat9, mri1 and mri2) for which no error is flagged but the returned residuals are clearly too large when compared with those computed by the other solvers. Although a direct solver such as HSL_MA77 [57] that allows the main work arrays and the matrix factors to be held out of core can extend the size of problem that can be solved, such solvers can be significantly slower. Thus there is a clear need for iterative solvers that require less memory.

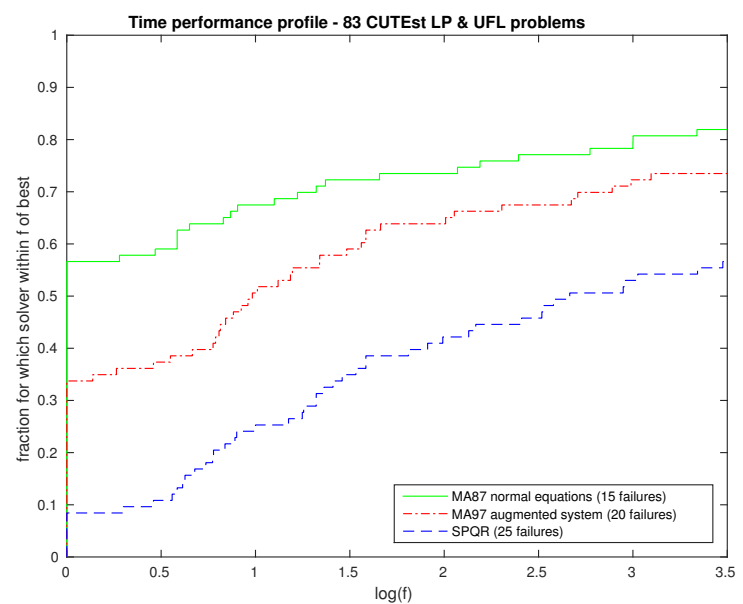

Fig. 1. Time performance profile for the direct solvers HSL_MA87, HSL_MA97 and SuiteSparseQR (SPQR) for test set $\mathcal{T}$. 

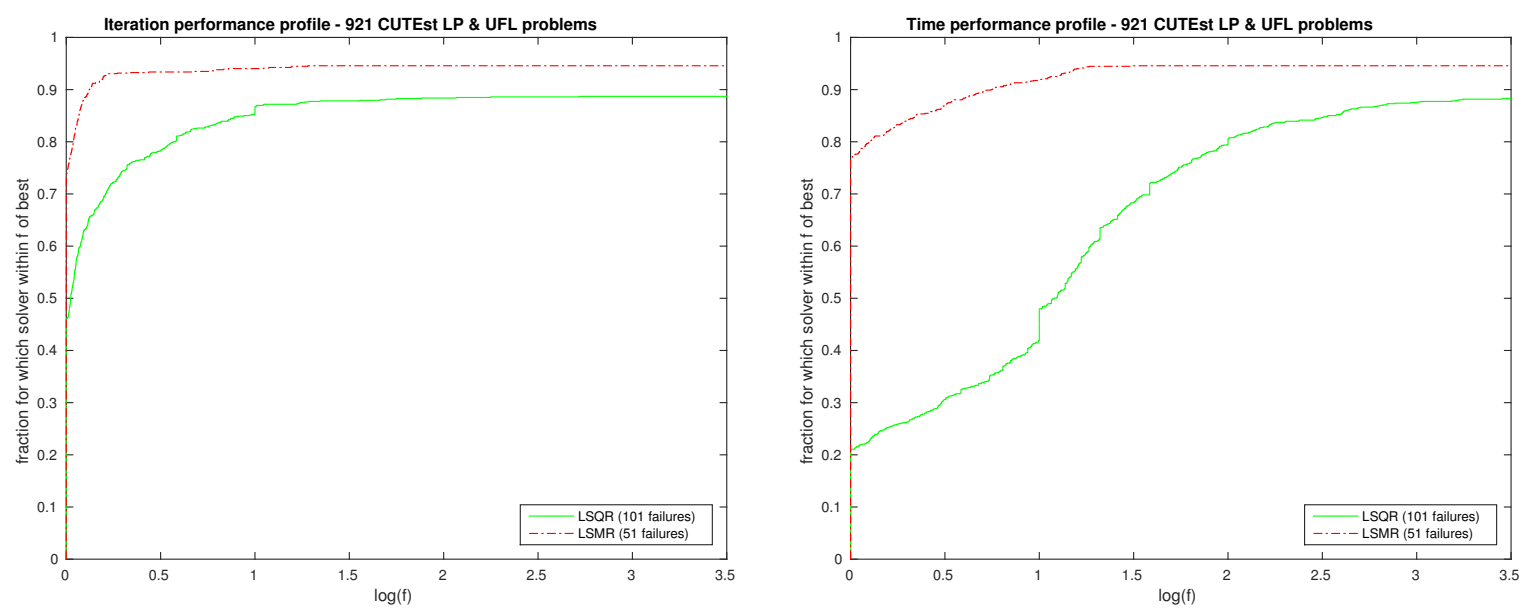

Fig. 1. Iteration performance profile (left) and time performance profile for LSMR and LSQR (right) for the complete CUTEst and UFL test set of 921 eligible problems with no preconditioning.

\section{LSQR VS LSMR}

CGLS (or CGNR) [35] is a long-established extension of the conjugate gradient method (CG) to least-squares problems. It is mathematically equivalent to applying CG to the normal equations, without actually forming them. The well-known and widely used LSQR algorithm of Paige and Saunders [53; 54] is algebraically identical to CGLS and, as shown in [11], both achieve similar final accuracy consistent with numerical stability. LSQR is based on the Golub-Kahan bidiagonalization of $A$ and has the property of reducing $\left\|r_{k}\right\|_{2}$ monotonically, where $r_{k}=b-A x_{k}$ is the residual for the approximate solution $x_{k}$.

The more recent LSMR algorithm of Fong and Saunders [24] is similar to LSQR in that it too is based on Golub-Kahan bidiagonalization of $A$. However, in exact arithmetic LSMR is equivalent to MINRES [52] applied to the normal equations, so that the quantities $\left\|A^{T} r_{k}\right\|_{2}$ (as well as, perhaps more surprisingly, $\left\|r_{k}\right\|_{2}$ ) are monotonically decreasing. Fong and Saunders report that LSMR may be a preferable solver because of this and because it may be able to terminate significantly earlier. Observe that if right-preconditioning with preconditioner $M$ is employed, then $\left\|\left(A M^{-1}\right)^{T} r\right\|_{2}$ is monotonic decreasing.

The implementation of LSMR used in this paper is a slightly modified version of the 2010 one of Fong and Saunders. The modifications include using allocatable arrays rather than automatic arrays (the latter can cause the code to crash with a segmentation fault error if the problem is large whereas allocated arrays allow memory failures to be captured and the code to be terminated with a suitable error flag set). More importantly, we incorporate a reverse communication interface that allows greater flexibility in how the user performs matrix vector products $A x$ and $A^{T} x$ and applies the (optional) preconditioner as well as enabling us to use our stopping criteria $\mathrm{C} 1$ and $\mathrm{C} 2$ (independently of the preconditioner used). The same modifications are made to LSQR for our tests. Both the modified version of LSMR and the Fong and Saunders code are available from http://web.stanford.edu/group/SOL/download.html.

In Figure 1, we present an iteration performance profile and a time performance for LSQR and LSMR with no preconditioning on the entire CUTEst and UFL set of 921 eligible examples. We see that LSMR has fewer failures compared to LSQR and requires a smaller number of iterations, which results in faster execution time. This 

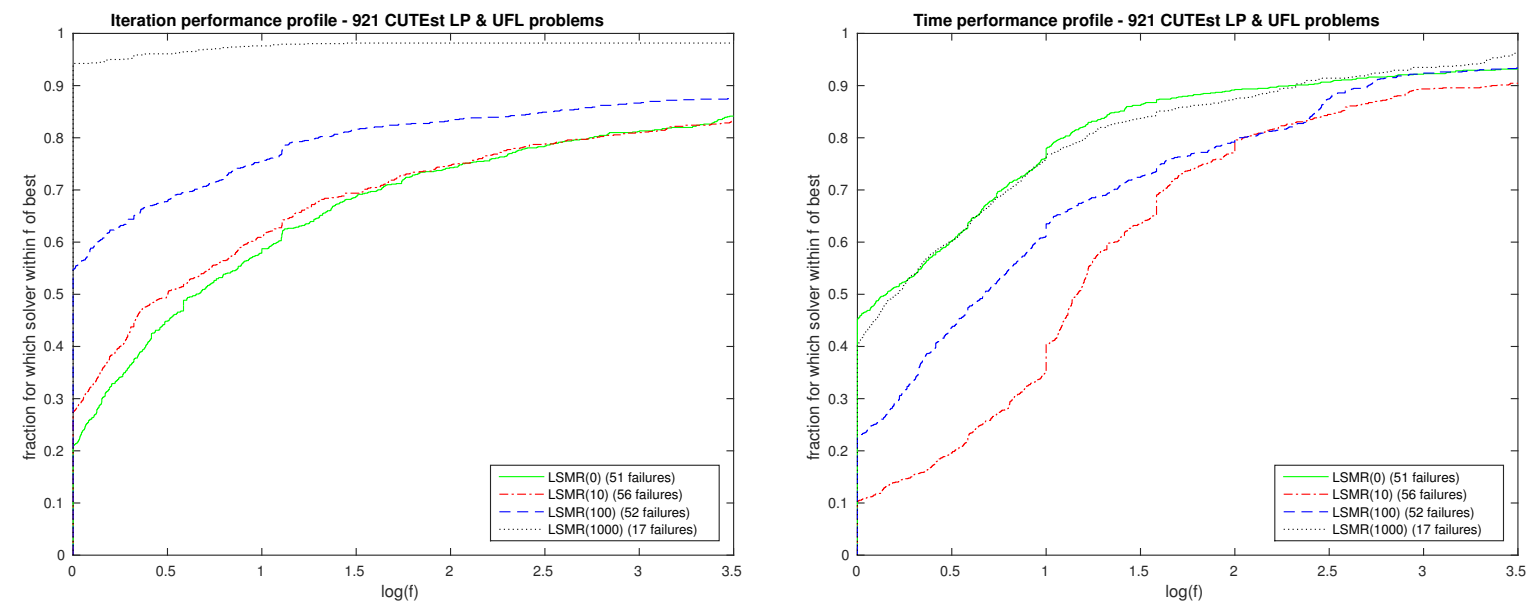

Fig. 2. Iteration performance profile (left) and time performance profile for LSMR with a range of values of localsize for the complete CUTEst and UFL test set of 921 eligible problems with no preconditioning.

confirms the findings of Fong and Saunders; in the remainder of this study we will limit our attention to LSMR.

Fong and Saunders propose incorporating local reorthogonalization in which each new basis vector is reorthogonalized with respect to the previous localSize vectors, where localSize is a user specified parameter. Setting localsize to 0 corresponds to no reorthogonalization while setting localsize to $n$ gives full reorthogonalization. Fong and Saunders report iteration counts for two linear programming problems with localsize set to $0,5,10,50$ and $n$. These illustrate that, compared with no reorthogonalization, setting localsize $=10$ or 50 can lead to a worthwhile reduction in the number of iterations for convergence but, as expected, more iterations are needed than for full reorthogonalization. Note that as $n$ vectors of size localsize are needed, for large problems full reorthogonalization is impractical both in terms of the computational time and memory requirements.

To examine the effect of localSize on our much larger test set, an iteration performance profile and a time performance profile for localsize set to $0,10,100$ and 1000 are given in Figure 2 (no preconditioning). We see that using a large value for localsize can significantly reduce the number of iterations and improve the success rate; the disadvantage is that the cost of each iteration (in terms of time and memory) increases with localsize.

\section{PRECONDITIONING STRATEGIES FOR NORMAL EQUATIONS}

In this section, we first consider a number of ways to choose the preconditioner $M$ for use with LSMR.

\subsection{Diagonal preconditioning}

The simplest form of preconditioning is diagonal preconditioning in which we solve

$$
\min _{y}\|b-A S y\|_{2}, \quad x=S y,
$$

where $S$ is a diagonal matrix that scales the columns of $A$ to give each unit 2norm. This requires only the diagonal entries of the normal matrix $C$ to be computed or, equivalently, the squares of the 2 -norms of the columns of $A$. This can be done 
in parallel, making the computation of the preconditioner and its application both straightforward and efficient (in terms of time and memory).

\subsection{Incomplete Cholesky factorizations}

Incomplete Cholesky (IC) factorizations have long been an important tool in the armoury of preconditioners for the numerical solution of large sparse, symmetric positive definite linear systems of equations; for an introduction and overview see, for example, [7; 60;67] and the long lists of references therein. Since (if $A$ has full column rank) the coefficient matrix $C$ of the normal equations (2) is positive definite, an obvious choice for the preconditioner is an IC factorization of $C$.

An IC factorization takes the form $L L^{T}$ in which some of the fill entries (entries in $L$ that were zero in the system matrix $C$ ) that would occur in a complete factorization are ignored. The preconditioned normal equations become

$$
\left(A L^{-T}\right)^{T}\left(A L^{-T}\right) y=L^{-1} C L^{-T} y=L^{-1} A^{T} b, \quad y=L^{T} x .
$$

Performing preconditioning operations involves solving triangular systems with $L$ and $L^{T}$. Over the years, a wealth of different variants have been proposed, including structure-based methods, those based on dropping entries below a prescribed threshold and others that prescribe the maximum number of entries allowed in $L$. We employ the recent limited memory approach of Scott and Tưma [66; 67] that exploits ideas from the positive semidefinite Tismenetsky-Kaporin modification scheme [44; 71]. The basic scheme employs a matrix factorization of the form

$$
C=(L+\hat{L})(L+\hat{L})^{T}-E,
$$

where $L$ is the lower triangular matrix with positive diagonal entries that is used for preconditioning, $\hat{L}$ is a strictly lower triangular matrix with small entries that is used to stabilize the factorization process but is subsequently discarded, and $E$ has the structure

$$
E=\hat{L} \hat{L}^{T}
$$

for details, see $[66 ; 67]$. The user specifies the maximum number of entries in each column of $L$ and $\hat{L}$. At each step of the factorization, the largest entries are kept in the current column of $L$, the next largest in the current column of $\hat{L}$, and the remainder are dropped. In practice, $C$ is optionally preordered and scaled and, if necessary, shifted to avoid breakdown of the factorization (which occurs if a non positive pivot is encountered) [47]. Thus the $L L^{T}$ incomplete factorization of the matrix

$$
\bar{C}=S Q^{T} C Q S+\alpha I
$$

is computed, where $Q$ is a permutation matrix chosen on the basis of sparsity, $S$ is a diagonal scaling matrix and $\alpha$ is a non-negative shift. It follows that $M=\overline{L L}^{T}$ with $\bar{L}=Q S^{-1} L$ is the incomplete factorization preconditioner.

When used to compute an incomplete factorization of the normal equations, there is no need to form and store all of $C$ explicitly; rather, the lower triangular part of its columns can be computed one at a time, used to perform the corresponding step of the incomplete Cholesky algorithm and then discarded. Of course, forming the normal matrix, even piecemeal, can entail a significant overhead (particularly if $m$ and $n$ are large and if $A$ has one or more dense rows) and potentially may lead to a severe loss of information in very ill-conditioned cases. 


\subsection{MIQR}

An alternative to an incomplete Cholesky factorization of $C$ is an approximate orthogonal factorization of $A$. If

$$
A \approx Q\left[\begin{array}{c}
R \\
0
\end{array}\right],
$$

where $Q$ is orthogonal and $R$ is upper triangular, then $C=A^{T} A \approx R^{T} R$ and, $M=R^{T} R$ can be used as a preconditioner. Again, applying the preconditioner involves triangular solves. Observe that the factor $Q$ is not used. There have been a number of approaches based on incomplete orthogonal factorizations of $A$ [4; 5; 41; 55; 59; 72]. Most recently, there is the Multilevel Incomplete QR (MIQR) factorization of Li and Saad [46].

When $A$ is sparse, many of its columns are likely to be orthogonal because of their structure. These structurally orthogonal columns form an independent set $S$. Once $S$ is obtained, the remaining columns of $A$ are orthogonalized against the columns in $S$. Since the matrix of remaining columns will in general still be sparse, it is natural to recursively repeat the process until the number of columns is small enough to orthogonalize with standard methods, or a prescribed number of reductions (levels) has been reached, or the matrix cannot be reduced further. This process results in a $Q R$ factorization of a column-permuted $A$ and forms the basis of the MIQR factorization. In practice, the $Q R$ factorization causes significant fill-in and so MIQR improves sparsity by relaxing the orthogonality and applying dropping strategies.

The MIQR algorithm does not require the normal matrix $C$ to be computed explicitly; only one row of $C$ is needed at any given time. Moreover, since $C$ is symmetric, only its upper triangular part (i.e., the inner products between the $i$-th column of $A$ and columns $i+1$ to $n$ ) needs to be calculated.

\subsection{RIF}

The RIF (Robust Incomplete Factorization) algorithm of Benzi and Tůma [8; 9] computes an LDLT factorization of the normal matrix $C$ without forming any entries of $C$, working only with $A$. The method is based on $C$-orthogonalization, i.e., orthogonalization with respect to the $C$-inner product defined by

$$
\langle x, y\rangle_{C}:=x^{T} C y=(A x)^{T}(A y) \text { for all } x, y \in \mathbb{R}^{n} .
$$

Assuming $A$ is of full column rank, $C$ is symmetric positive definite and this then provides an inner product on $\mathbb{R}^{n}$. Given the $n$ linear independent vectors $e_{1}, e_{2}, \ldots, e_{n}$ ( $e_{i}$ is the $i$-th unit basis vector), a $C$-orthogonal set of vectors $z_{1}, z_{2}, \ldots, z_{n}$ is built using a Gram-Schmidt process with respect to (2). This can be written in the form

$$
Z^{T} C Z=D=\operatorname{diag}\left(d_{1}, d_{2}, \ldots, d_{n}\right),
$$

where $Z=\left[z_{1}, z_{2}, \ldots, z_{n}\right]$ is unit upper triangular and the $d_{i}$ are positive. It follows that $Z^{T}=L^{-1}$, where $L$ is the unit lower triangular factor of the root-free Cholesky factorization $C=L D L^{T}$. It can be shown [8] that the $L$ factor can be obtained as a by-product of the $C$-orthogonalization process at no extra cost.

Two different types of preconditioner can be obtained by carrying out the $C$ orthogonalization process incompletely. The first drops small entries from the computed vectors as the $C$-orthogonalization proceeds, resulting in a sparse matrix $\tilde{Z} \approx L^{-T} ;$ that is, an incomplete inverse factorization of $C$ of the form

$$
C^{-1} \approx \tilde{Z} \tilde{D}^{-1} \tilde{Z}^{T},
$$

where $\tilde{D}$ is diagonal with positive entries, is computed. This is a factored sparse approximate inverse that can be used as a preconditioner for the CG algorithm applied 
to the normal equations. The preconditioner is guaranteed to be positive definite and can be applied in parallel since its application requires only matrix-vector products. It is generally known as the stabilized approximate inverse (SAINV) preconditioner.

The second approach (the RIF preconditioner) is obtained by discarding the computed sparsified vector $\tilde{z}_{i}$ as soon as it has been used to form the corresponding parts of the incomplete factor $\tilde{L}$ of $C$. This gives an algorithm for computing an incomplete Cholesky factorization for the normal equations

$$
C \approx \tilde{L} \tilde{D} \tilde{L}^{T}
$$

Again, the preconditioner $M=\tilde{L} \tilde{D} \tilde{L}^{T}$ is positive definite and (in exact arithmetic) breakdown during its computation is not possible. An important feature of the RIF preconditioner is that it incurs only modest intermediate storage costs, although implementing the algorithm for its construction so as to exploit the sparsity of $A$ is far from straightforward (see [9] for a brief discussion). Benzi and Tủma report that the RIF preconditioner is generally more effective at reducing the number of CG iterations than the SAINV one and is thus the one included in this study. Over the past few years, a number of papers on preconditioners for least-squares problems have used RIF as a benchmark, but these papers limit their reported tests to a small number of examples $[3 ; 12 ; 46 ; 48]$.

\section{BA-GMRES}

The BA-GMRES method for solving least-squares problems combines using a stationary inner iteration method with the Krylov subspace method GMRES [61] applied to the normal equations. For problems for which convergence is slow and for very large problems for which storage is an issue, restarted GMRES is used. In contrast to the other methods discussed so far, rather than forming an explicit preconditioner, a number of steps of a stationary iterative method are applied within the GMRES algorithm whenever an application of the preconditioner is needed. Such techniques are often called inner-outer iteration methods. While the basic idea is not new, it has recently been explored in the context of least-squares problems by Hayami et al. [34; 48; 49]. In particular, for overdetermined least-squares problems, they propose using Jacobi- (Cimmino [16]) and SOR-type (Kaczmarz [43]) iterative methods as inner-iteration preconditioners for GMRES and advocate their so-called BA-GMRES approach for the efficient solution of rank-deficient problems. Jacobi iterations can be advantageous for parallel implementations but in this study, we limit our attention to serial implementations and use SOR iterations with automatic selection of the relaxation parameter $\omega$ as described in [48; 49].

BA-GMRES corresponds to GMRES applied to

$$
\min _{x}\|B b-B A x\|_{2}
$$

where the rectangular matrix $B \in \mathbb{R}^{n \times m}$ is the (left) preconditioner. Morikuni and Hayami [48; 49] show that if $B$ satisfies $\mathcal{R}(A)=\mathcal{R}\left(B^{T}\right)$ and $\mathcal{R}\left(A^{T}\right)=\mathcal{R}(B)$, the solution of (4) is also a solution of the least-squares problem (1). $B$ is not formed or stored explicitly. Instead, at each GMRES iteration $k$, when preconditioning is needed a fixed number of steps of a stationary iterative method are applied to a system of the form

$$
A^{T} A z=A^{T} A v_{k}
$$

to obtain $z$ for a given $v_{k}$, which is used to compute the next GMRES basis vector $v_{k+1}$. Thus at each GMRES iteration, another system of normal equations is solved approximately using a stationary iterative method and this can be done 
without forming any entries of $A^{T} A$ explicitly (see [60], Section 8.2 for details); all that is required are repeated matrix-vector products with $A$ and $A^{T}$. This allows nonsymmetric preconditioning for least-squares problems. Another potential advantage of BA-GMRES is that it avoids forming and storing an incomplete factorization; the memory used is determined solely by the number of steps of GMRES that are applied before restarting.

Morikuni and Hayami observe that inner iteration preconditioners can also be applied to CGLS and LSMR. This has the merit of using only short-term recurrences and so the memory requirements are less than for BA-GMRES. The results reported in [48; 49] for a small set of test problems (including rank-deficient examples) indicate faster times, fewer iterations and greater robustness using BA-GMRES; thus BAGMRES (for which software is available, see Section 8.5) is used in this study.

\section{PRECONDITIONING STRATEGIES FOR THE AUGMENTED SYSTEM}

An alternative to preconditioning the normal equations is to precondition the augmented system (3). In some applications, preconditioning the augmented system is advocated when the normal equations are highly ill-conditioned (see, for instance, [51]). A number of possible approaches exist, including employing an incomplete factorization designed for general indefinite symmetric systems or a signed incomplete Cholesky factorization [68] designed specifically for systems of the form (3). Chow and Saad [15] considered the class of incomplete LU preconditioners for solving indefinite problems and later $\mathrm{Li}$ and Saad [45] integrated pivoting procedures with scaling and reordering. Building on this, Greif, He, and Liu [32] recently developed a new incomplete factorization package SYM-ILDL for general sparse symmetric indefinite matrices. The factorization is of the form

$$
K \approx L D L^{T},
$$

where $L$ is unit lower triangular and $D$ is block diagonal, with $1 \times 1$ and $2 \times 2$ blocks on the diagonal (corresponding to $1 \times 1$ and $2 \times 2$ pivots). For SYM-ILDL, $K$ may be any sparse indefinite matrix; no advantage is made of the specific block structure of (3). Independently, Scott and Tůma [69] report on the development of incomplete factorization algorithms for symmetric indefinite systems and propose a number of new ideas with the goal of improving the stability, robustness and efficiency of the resulting preconditioner. The SYM-ILDL software is available [32]. It is written in $\mathrm{C}++$ and is designed either to be called from within MATLAB or from the command line. The user may save the computed factor data to a file but (when used from the command line) the package offers no procedure to take that data and use it as a preconditioner. Without substantial further work to set up a more flexible and convenient user interface, we were restricted to running individual problems one at a time. We performed limited experiments using SYM-ILDL (see also [68; 69]). These demonstrated that there are least-squares problems for which SYM-ILDL is able to provide an effective preconditioner but for other problems we were unsuccessful in obtaining the required least-squares solution. The prototype code of Scott and Tuma likewise gave very mixed results. We conclude that further work is needed for these codes to be useful for least-squares problems; they are not explored further in this study.

For matrices $K$ of the augmented form (3), Scott and Tůma [68] propose extending their limited memory IC approach to a limited memory signed incomplete Cholesky factorization of the form (5) where $L$ is a lower triangular matrix with positive diagonal entries and $D$ is a diagonal matrix with entries \pm 1 . In practice, an $L D L^{T}$ factorization 
of

$$
\bar{K}=S Q^{T} K Q S+\left[\begin{array}{ll}
\alpha_{1} I & \\
& -\alpha_{2} I
\end{array}\right]
$$

is computed, where $Q$ is a permutation matrix, $S$ is a diagonal scaling matrix, and $\alpha_{1}$ and $\alpha_{2}$ are non-negative shifts chosen to prevent breakdown. The preconditioner is $M=\bar{L} D \bar{L}^{T}$, with $\bar{L}=Q S^{-1} L$. In this case, the permutation $Q$ is chosen not only on the basis of sparsity but also so that a variable in the $(2,2)$ block of $K$ is not ordered ahead of any of its neighbours in the $(1,1)$ block; see [68] for details of this so-called constrained ordering.

An important advantage of a signed IC factorization over a general indefinite incomplete factorization is its simplicity in that it avoids the use of numerical pivoting. If we use the natural order $(Q=I)$, the factorization becomes

$$
K \approx\left[\begin{array}{cc}
I & \\
L_{1} & L_{2}
\end{array}\right]\left[\begin{array}{cc}
I & -I
\end{array}\right]\left[\begin{array}{cc}
I & L_{1}^{T} \\
& L_{2}^{T}
\end{array}\right]
$$

and so

$$
L_{1} \approx A^{T} \text { and } L_{1} L_{1}^{T} \approx L_{2} L_{2}^{T} .
$$

If we choose $L_{1}=A^{T}$ then this reduces to an IC factorization of the normal equations. However, by choosing $L_{1} \neq A^{T}$ or $Q \neq I$, this approach can exploit the structure of the augmented system while avoiding the normal equations.

As the signed IC preconditioner is indefinite, a general non symmetric iterative method such as GMRES [61] is needed; we use right preconditioned restarted GMRES. Since GMRES is applied to the augmented system matrix $K$, the stopping criteria is applied to $K$. With the available implementations of GMRES, it is not possible during the computation to check whether either of the stopping conditions $\mathrm{C} 1$ or $\mathrm{C} 2$ (which are based on $A$ ) is satisfied; they can, of course, be checked once GMRES has terminated. Instead, we use the scaled backward error

$$
\frac{\left\|K y_{k}-c\right\|_{2}}{\|c\|_{2}}<\delta_{3}
$$

where $y_{k}$ is the computed solution on the $k$ th step. In our experiments we set $\delta_{3}=10^{-6}$.

If we want to use a solver that is designed for symmetric indefinite systems, in place of GMRES we can use MINRES [52]. However, MINRES requires a positive definite preconditioner and so we use $M=\overline{L L}^{T}$, that is, we replace the entries -1 in $D$ by +1 so that $D$ becomes the identity. Again, the stopping conditions C1 or C2 cannot be checked inside MINRES and we use instead (6).

\section{PRECONDITIONING SOFTWARE AND PARAMETER SETTINGS}

\subsection{Diagonal preconditioning}

In Figure 3 we present iteration and time performance profiles for LSMR with diagonal preconditioning using a range of values for the LSMR reorthogonalization parameter localSize. A large value reduces the iteration count but increases the time (and memory) required (so that a number of problems exceed the time limit if localsize is set to 1000 , which accounts for the increase in the number of failures).

\subsection{IC preconditioner for normal equations}

A software package HSL_MI35 that implements the limited memory IC algorithm discussed in Section 5.2 for the normal equations has been developed for the HSL mathematical software library [40]. This code is a modified version of HSL_MI28 [66]. 

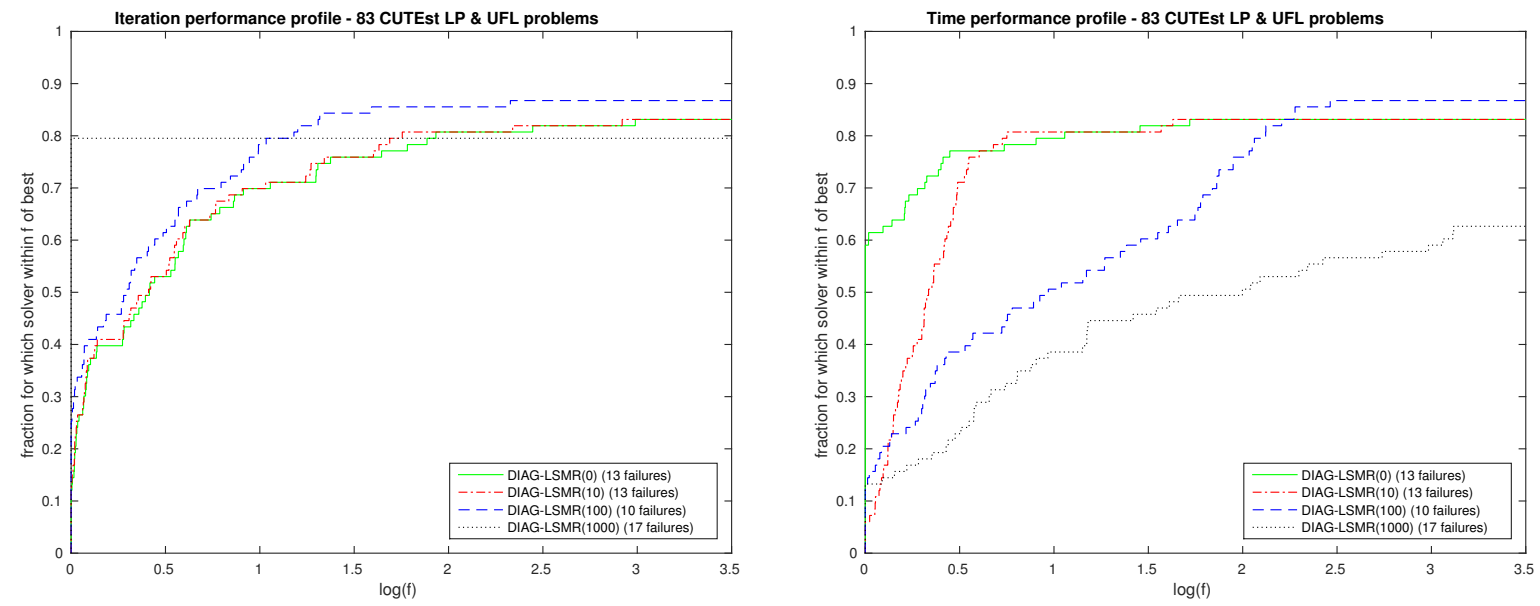

Fig. 3. Iteration performance profile (left) and time performance profile (right) for LSMR with diagonal preconditioning using a range of values of localsize for test set $\mathcal{T}$.

Modifications were needed to allow the user to specify the maximum number of entries allowed in each column of the incomplete factor $L$ (in HSL_MI28 the user specified the amount of fill allowed but as columns of $C$ may be dense, or close to dense, this change was needed to keep $L$ sparse). In addition, the user may either supply the matrix $A$ and call a subroutine within the package to form $C$ explicitly or, to save memory, $A$ may be passed directly to the factorization routine. In this case, the lower triangular part of each column of the (permuted) normal matrix is computed as needed during the factorization (although the sparsity pattern of $C$ is computed if reordering is selected). Note that, if $A$ and not $C$ is supplied, the range of scaling options is restricted since the equilibration and maximum matching-based scalings that are offered through the use of the packages MC77 [58] and MC64 [21;22], respectively, require $C$ explicitly. The default scaling is $l_{2}$ scaling, in which column $j$ of $C$ is normalised by its 2 -norm; this needs only one column of $C$ at a time. We observe that HSL_MI35 is designed to solve the weighted least-squares problem but in our tests the weights are set to one.

The parameters lsize and rsize respectively control the maximum number of entries in each column of $L$ and each column of the matrix $\hat{L}$ that is used in the computation of $L$ (recall (1)). Iteration and time performance profiles for LSMR preconditioned by HSL_MI35 using lsize $=$ rsize $=10$ and lsize $=$ rsize $=20$ are given in Figure 4. Here and elsewhere, the time used for the time performance profile are the total solution time (that is, the time to compute the preconditioner plus the time to run preconditioned LSMR). We see that the iteration count is reduced by increasing the number of entries allowed and as the time is not significantly adversely effected, 1 size $=$ rsize $=20$ is used in all other experiments with HSL_MI35.

In Figure 5 we present iteration and time performance profiles for LSMR preconditioned by HSL_MI35 using a range of values for the LSMR reorthogonalization parameter localSize. As expected, using a large value reduces the iteration count but increases the time (and memory) required; localsize $=10$ is used in all other experiments with HSL_MI35.

\subsection{MIQR}

The MIQR package available at http://www-users.cs.umn.edu/ saad/software/ is for solving least-squares systems by a preconditioned CGNR algorithm and is written 

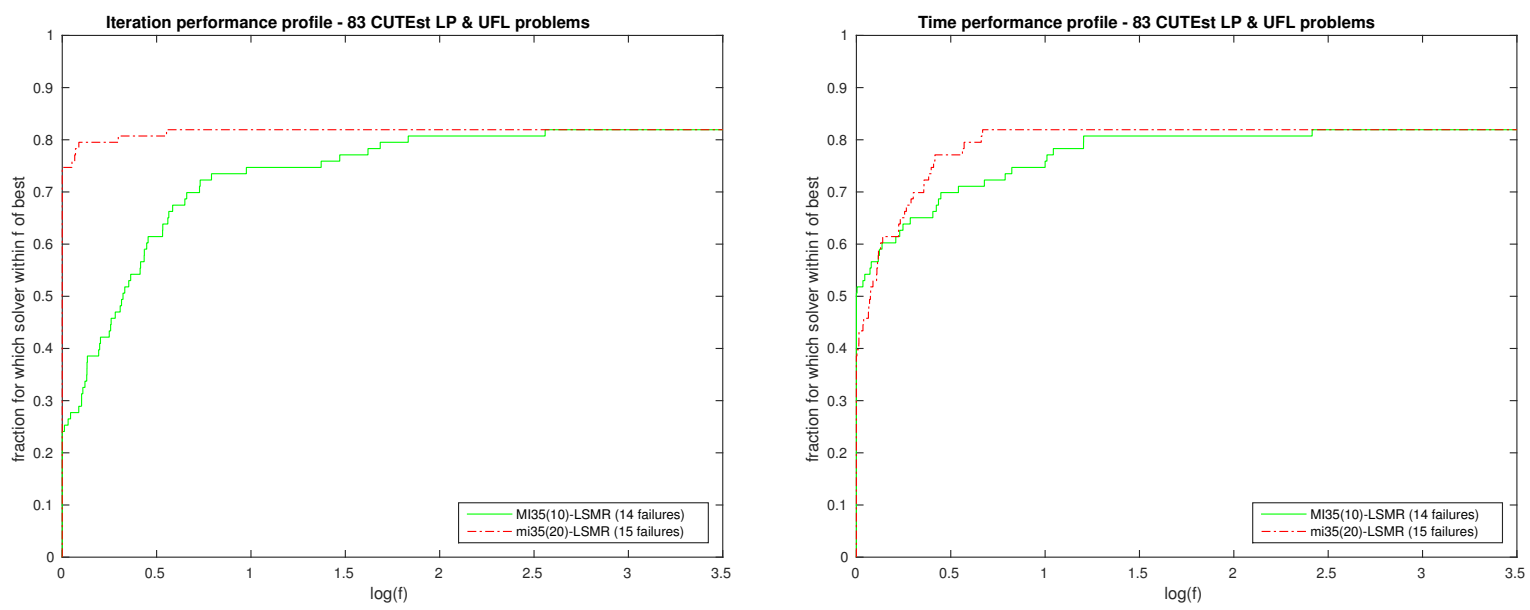

Fig. 4. Iteration performance profile (left) and time performance profile (right) for LSMR preconditioned by HSL_MI35 with lsize $=\operatorname{rsize}=10$ and lsize $=\operatorname{rsize}=20$ for test set $\mathcal{T}$.
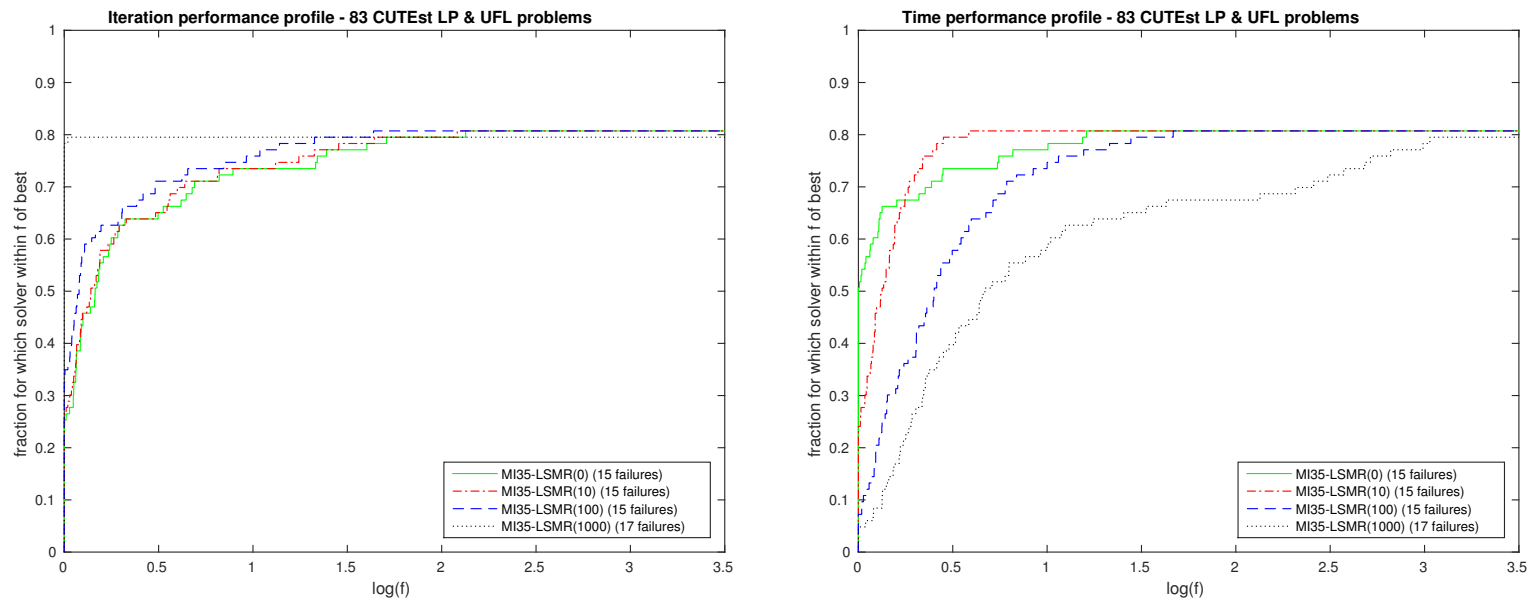

Fig. 5. Iteration performance profile (left) and time performance profile (right) for LSMR preconditioned by HSL_MI35 using a range of values of localsize for test set $\mathcal{T}$.

in C. As all our experiments are performed in Fortran, we have chosen to use a Fortran version of MIQR that is available from the GALAHAD optimization software library [27]. This is essentially a translation of Li and Saad [46]'s code, but with extra checks and features to make the problem data input easier. Key parameters, such as the maximum number of recursive levels of orthogonalization, the required angles between approximately orthogonal columns, the drop tolerance, and the maximum number of fills permitted per column, are precisely as given by $\mathrm{Li}$ and Saad.

Figure 7 presents iteration and time performance profiles for MIQR-preconditioned LSMR using a range of values of the reorthogonalization parameter localsize. The number of failures appears relatively insensitive to the choice of localsize but the iteration count decreases as localSize increases while using a value of 10 is the best in terms of time. 

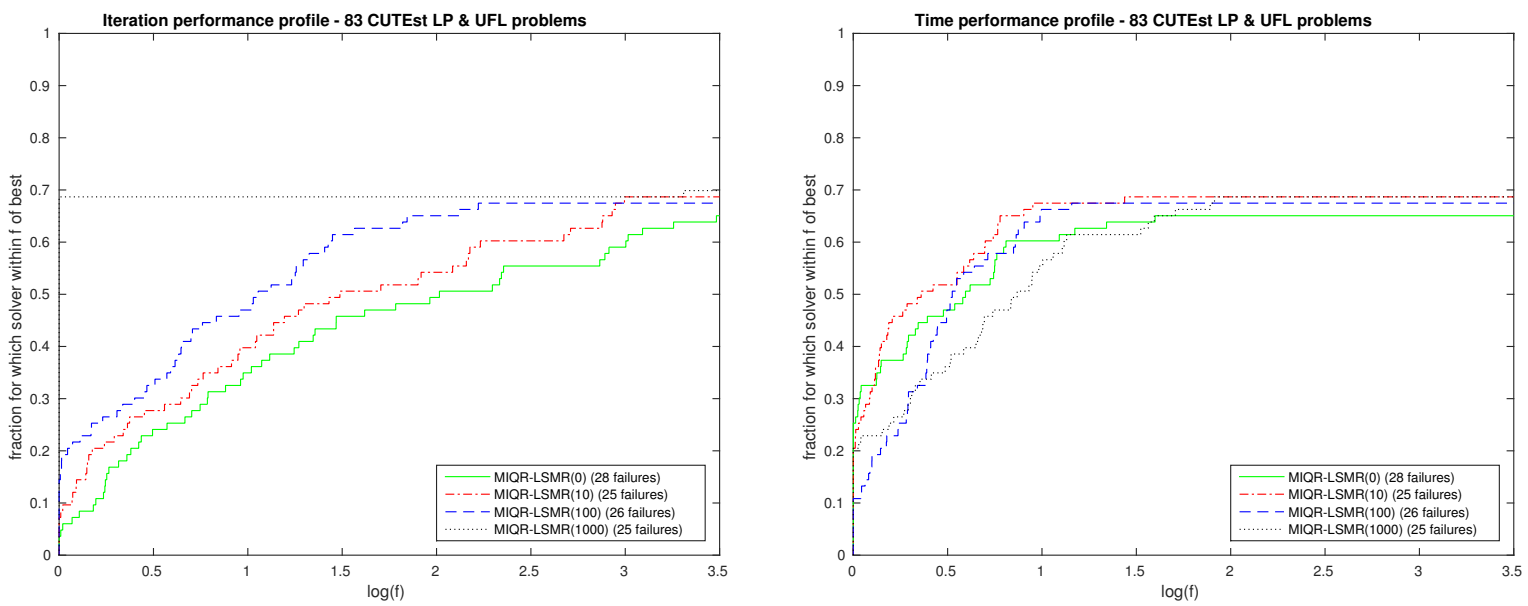

Fig. 6. Iteration performance profile (left) and time performance profile (right) for LSMR with MIQR preconditioning using a range of values of localsize for test set $\mathcal{T}$.

\subsection{RIF}

A right-looking implementation of RIF is available at http://www2.cs.cas.cz/ tuma/ sparslab.html. However, for our tests, Tůma provided a more recent left-looking version (see [70] for details of the right- and left-looking variants). The latter works only with $A$ and $A^{T}$ and has the advantage that the required memory can be computed before the factorization begins using a symbolic preprocessing step [70]. As full documentation for the software is lacking, we relied on Túma for advice on the parameter settings; in particular, we used absolute dropping with a drop tolerance of 0.1. In Figure 7, we give iteration and time performance profiles for RIFpreconditioned LSMR using a range of values of the reorthogonalization parameter localSize. There is no uniformly best value; in the rest of our experiments, we set localsize to 10 .
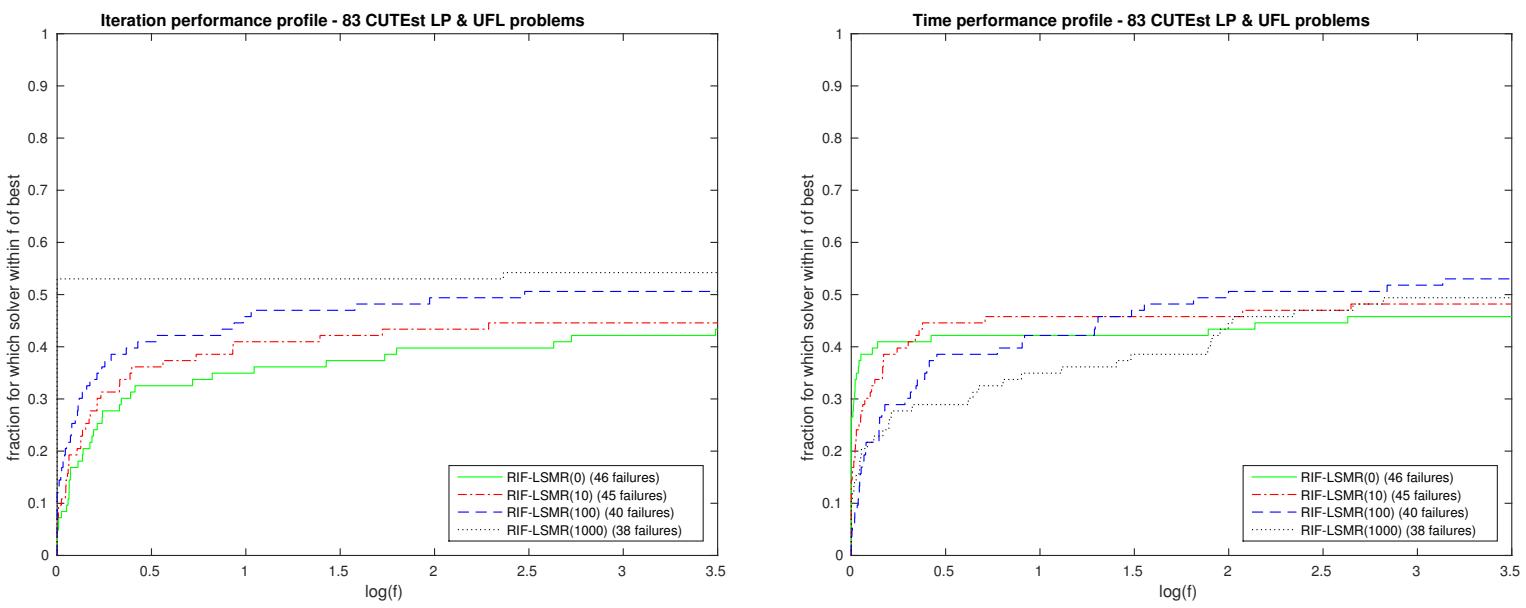

Fig. 7. Iteration performance profile (left) and time performance profile (right) for LSMR with RIF preconditioning using a range of values of localsize for test set $\mathcal{T}$. 


\subsection{BA-GMRES}

There are codes for the BA-GMRES method preconditioned by NR-SOR inner iterations developed by Morikuni available at http://researchmap.jp/KeiichiMorikuni/ Implementations (March 2015). However, these are not in the form that we can readily use for large-scale testing purposes. In particular, they employ automatic arrays (and will thus fail for a very large problem for which there is insufficient memory) and they contain "stop" statements (so again, they can fail without prior warning). As a result, we implemented a modified version of BA-GMRES. This also allowed us to use the stopping criteria $\mathrm{C} 1$ and $\mathrm{C} 2$ for consistency with the preconditioned LSMR tests (as in our tests with other methods, the time for computing the residuals needed for checking $\mathrm{C} 1$ and $\mathrm{C} 2$ at each iteration are excluded from the reported times).

As restarted GMRES is employed, the user must choose the number gmres_its of iterations between restarts. A compromise between a large value that reduces the overall number of iterations and a small value that limits the storage should be used. We performed some preliminary experiments to try and choose a suitable value to use for all our tests; our findings are in Figure 8. On the basis of these, we set gmres_its $=1000$. Note that if the number (iter) of iterations required for convergence is less than gmres_its, so that we do not unfairly overestimate the memory required, the reported memory for BA-GMRES is for gmres_its = iter. Following Morikuni, our
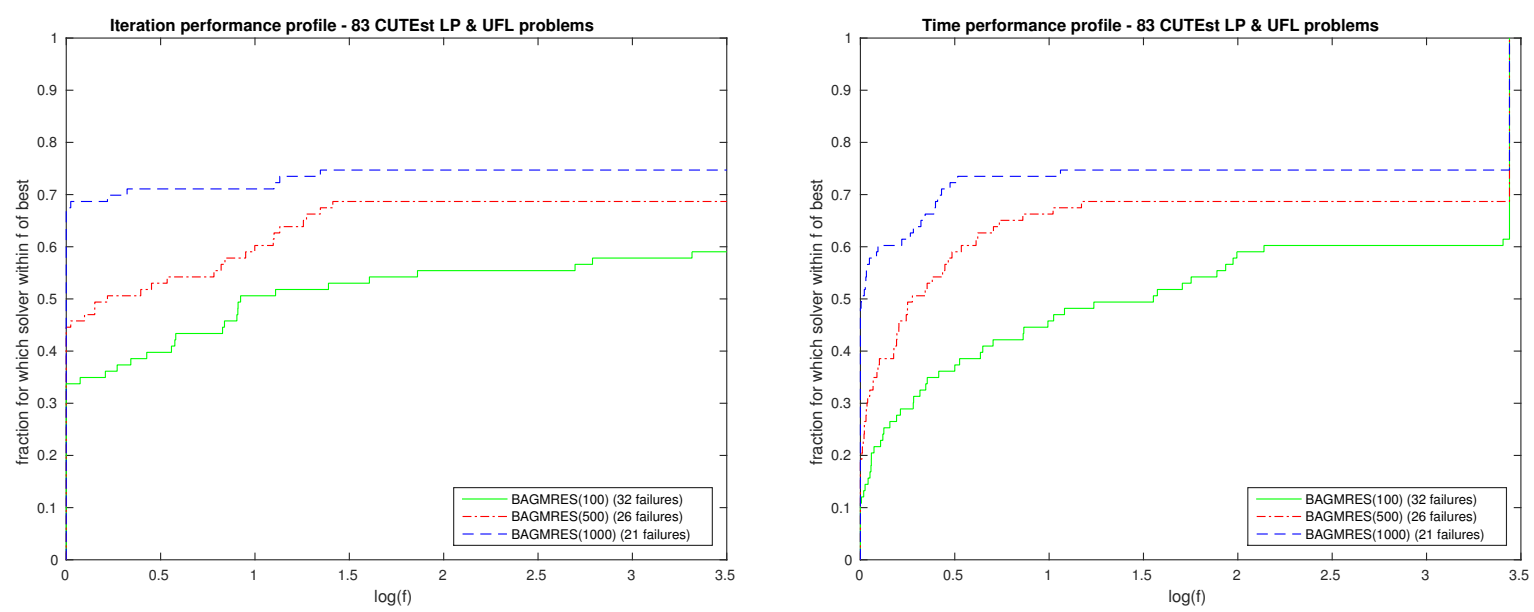

Fig. 8. Iteration performance profile (left) and time performance profile for BA-GMRES with different restart parameters for test set $\mathcal{T}$.

implementation of BA-GMRES allows the user to choose between using NR-SOR and Cimmino inner iterations. For the former, the user may supply the number of inner iterations and the relaxation parameter; otherwise, these are computed automatically using the procedure proposed in $[48 ; 49]$. We use NR-SOR inner iteration with automatic parameter selection in our tests.

\subsection{Signed IC preconditioner: augmented system}

A software package HSL_MI30 that implements the limited memory signed IC algorithm discussed in Section 7 for the augmented system is available within HSL; details are given in [68]. In our tests, we use the default settings for HSL_MI30 and the parameters lsize and size that control the number of entries in $L$ and the intermediate memory used to compute the factorization are both set to 20. For GMRES and MINRES we use 
the HSL implementations MI24 (with the restart parameter set to 1000) and HSL_MI32, respectively.

\section{BENEFITS OF SIMPLE PARALLELISM}

Having selected what we consider to be good parameter settings, a natural question is how much the methods in question might benefit from simple parallelism? Figure 9 illustrates the improvements possible simply by performing sparse matrix-vector products and triangular preconditioning solves on 4 rather than a single processor. Each preconditioner gains from parallelism, and perhaps unsurprisingly the most significant gains are for those methods for which (MKL-assisted) matrix-vector products and triangular solves dominate the solution time. Note that the serial experiments were necessarily repeated to obtain this data, since here we use MKL BLAS while our earlier experiments used the open BLAS. On the basis of these findings, our remaining comparisons use data from these runs on 4 processors.

\section{SOLVER COMPARISON RESULTS}

\subsection{Performance comparison for preconditioning LSMR}

Figure 10 presents iteration and time performance profiles for LSMR run both without preconditioning and with diagonal, MIQR, RIF and IC (HSLMI35) preconditioning run on 4 processors. Here we chose localsize $=0$ for no preconditioning and diagonal preconditioning and localsize $=10$ for MIQR, RIF and IC preconditioning since these appeared to give the best (time) performances in the individual preconditioner comparisons reported in Sections 4 and 8. We see that, in terms of iteration counts, the incomplete factorization is the best preconditioner but, in terms of time, the simplest option of diagonal preconditioning is slightly better than IC preconditioning (and has the advantages of needing minimal memory and being trivially parallelizable). The close time-ranking of the diagonal and IC preconditioners is confirmed in Figure 11. We observe that Morikuni and Hayami [48] also found diagonal preconditioning to give the fastest solution times in some of their tests.

In Figure 12 we compare the remaining three preconditioners. We see that in terms of time MIQR preconditioning is broadly similar to running without a preconditioner, and that the effects of a reduction in iteration counts for the former is balanced by the cost of computing and applying the preconditioner. This is reinforced in Figure 13 when RIF is removed from the picture.

The current implementation of RIF is somewhat slow. For problems for which the RIF preconditioner performs reasonably well (including the IG5-1x problems), more than $95 \%$ of the total solution time can be spent on computing the preconditioner, even though it can be significantly sparser than that computed using HSL_MI35 or MIQR. The uncompetitive construction time appears to be largely attributable to the searches performed to determine which $C$-inner products need to be computed; this is currently a subject of separate investigation [70]. For 21 of the 83 test problems, computing the RIF preconditioner exceeded our time limit of 600 seconds. Furthermore, for our test set $\mathcal{T}$ as a whole and the current settings, RIF is not especially effective. For the 62 problems for which the RIF preconditioner was successfully computed, 22 went on to exceed the LSMR iteration limit and a further 2 exceeded the total time limit. Again, this is consistent with [48]. We observe, however, that in many cases the RIF preconditioner is sparser than, for example, the IC preconditioner. Using a smaller drop tolerance may improve the quality at the cost of more fill but the time to compute the preconditioner can also increase significantly. 

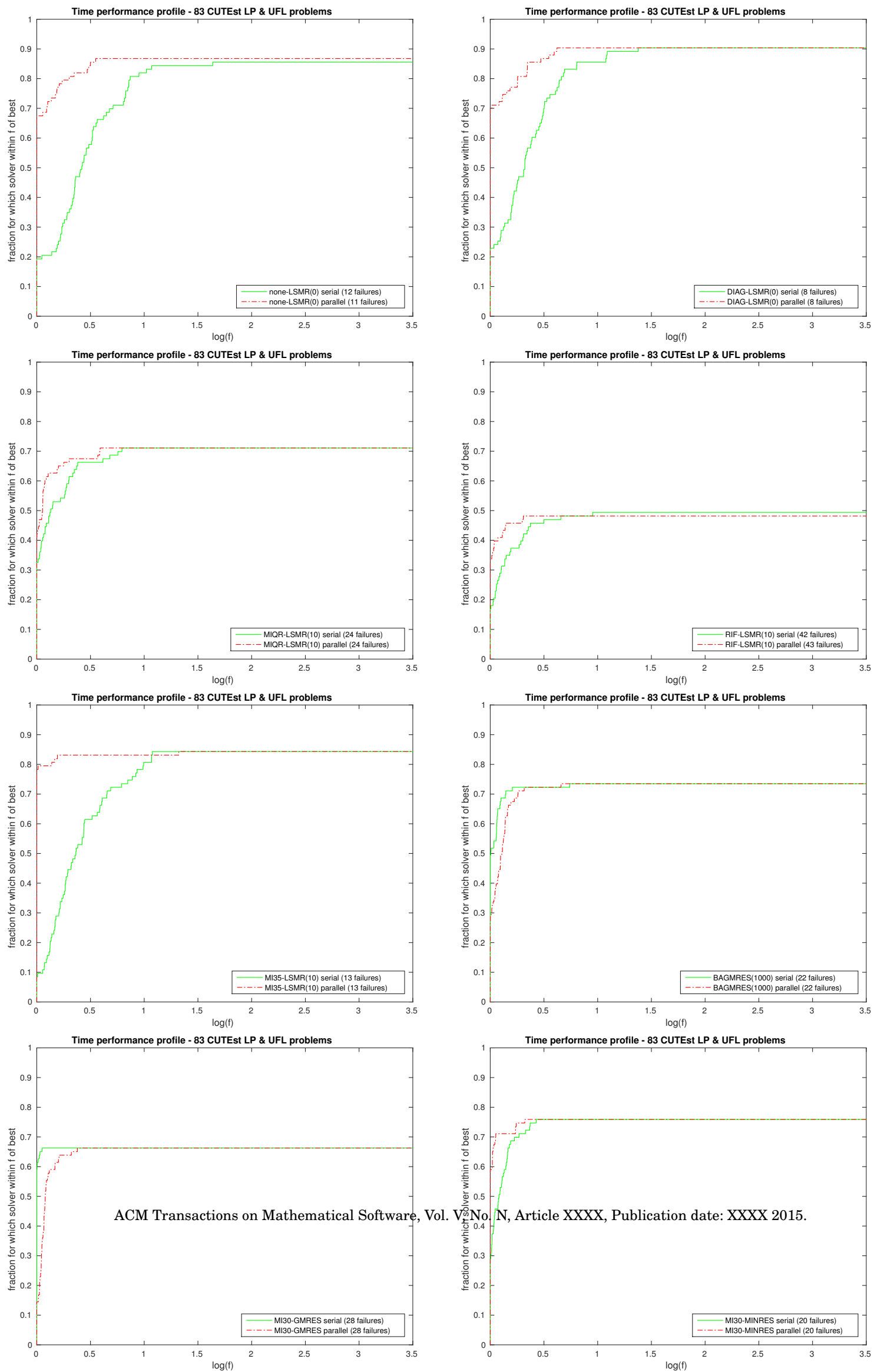

Fig. 9. Time performance profiles for serial and parallel execution of the methods described in Section 8 for test set $\mathcal{T}$. 

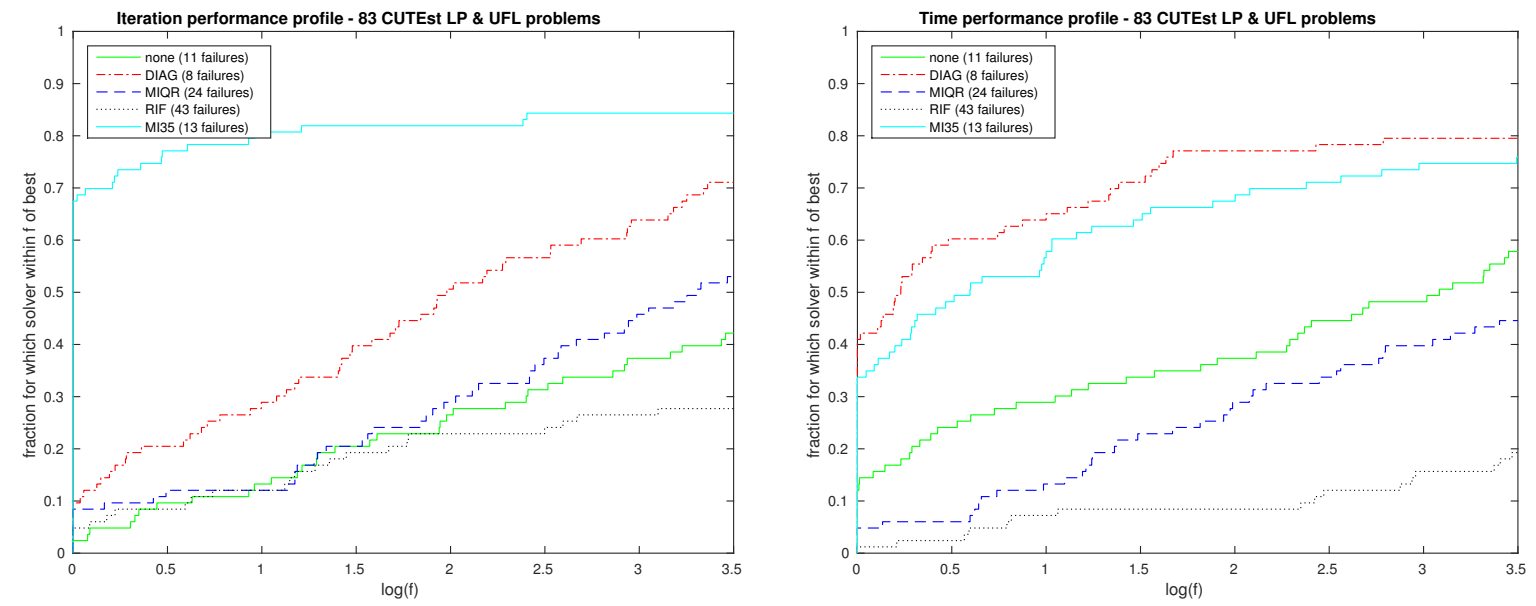

Fig. 10. Iteration performance profile (left) and time performance profile for different preconditioners used with LSMR for test set $\mathcal{T}$.

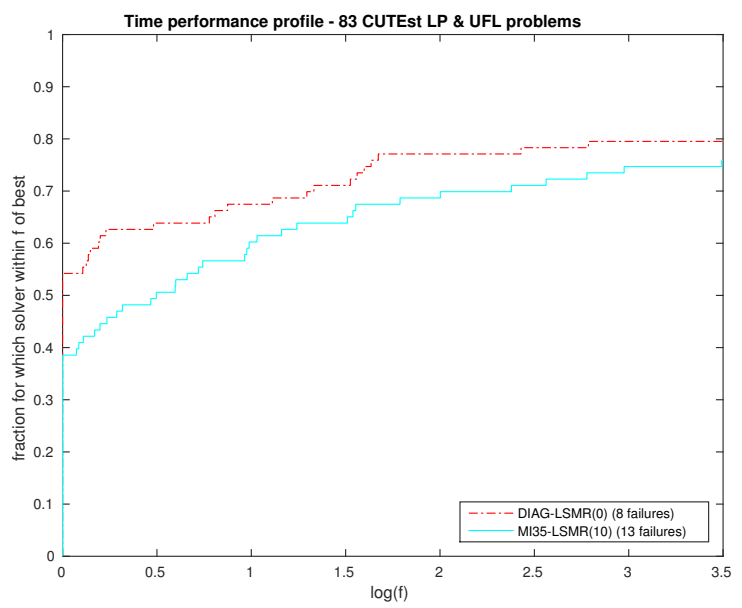

Fig. 11. Time performance profile for diagonal and IC preconditioners used with LSMR for test set $\mathcal{T}$.

\subsection{Performance comparison with BA-GMRES}

Time performance profiles for BA-GMRES are given in Figure 14. We see that, on our test set, BA-GMRES is slower than using LSMR with diagonal or IC preconditioning but is faster than LSMR with no preconditioning and MIQR preconditioning. However, a closer look at the results (see the summary tables given in the Appendix and [30]) shows that BA-GMRES is able to efficiently solve some examples that preconditioned LSMR and the direct solvers struggle with. In particular, BA-GMRES performs strongly on the GL7dxx problems and solves problem SPAL_004 in only one iteration. However, it is poor for the pseex problems.

\subsection{Performance comparison with signed incomplete factorization}

In Figure 15, time performance profiles are given for solving the augmented system using the signed incomplete Cholesky factorization preconditioner (HSL_MI30) run 

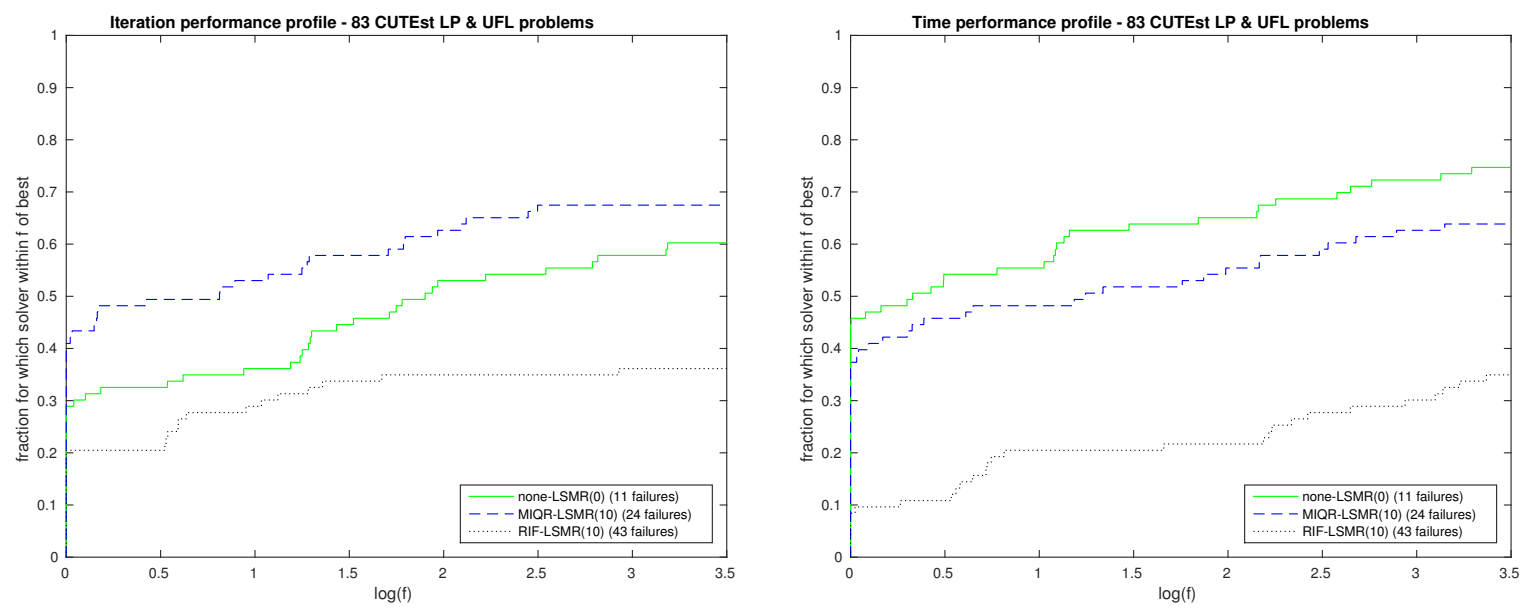

Fig. 12. Iteration and time performance profiles for LSMR with no preconditioning and MIQR and RIF preconditioning for test set $\mathcal{T}$.

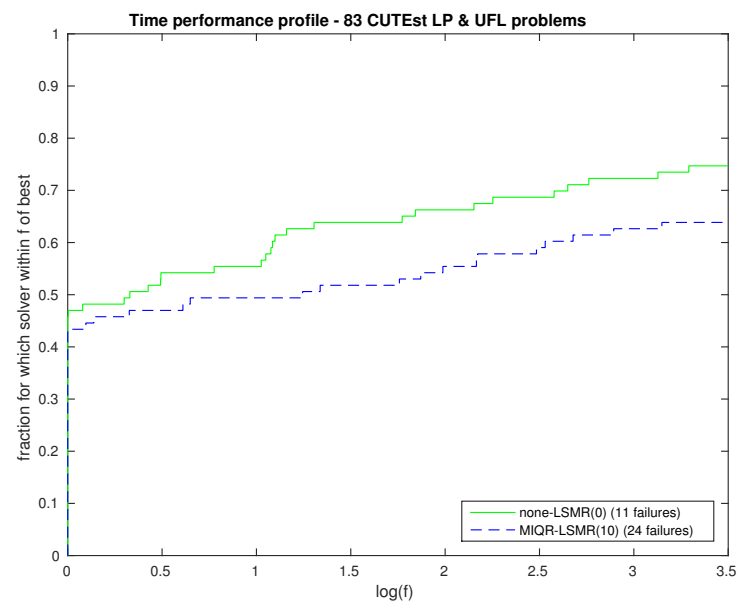

Fig. 13. Time performance profile for LSMR with no preconditioning and MIQR preconditioning for test set $\mathcal{T}$.

with GMRES(1000) and MINRES; the IC preconditioner (HSL_MI35) for the normal equations run with LSMR is also included. We see that HSL_MI35 preconditioned LSMR is faster than solving the augmented system and has the least number of failures. Note that the number of entries in the factors for the normal equations is approximately $n \times 1$ size whereas for the augmented system the number is bounded above by $m+n z(A)+(m+n) \times$ lsize (where $n z(A)$ is the number of entries in $A$ ). Thus when working with the augmented system each application of the preconditioner is considerably more expensive.

As observed in Section 7, for the signed incomplete factorization run with MINRES or GMRES, the stopping criteria is the scaled backward error for the augmented system (3) and thus conditions C1 and/or C2 may not be satisfied. For a significant portion of our test set, if $\delta_{3}$ in (6) is set to be $10^{-8}$ then either $\mathrm{C} 1$ or $\mathrm{C} 2$ is satisfied (see Tables 3.25 and 3.26 in [30]). Indeed, in some cases where we report a failure because 

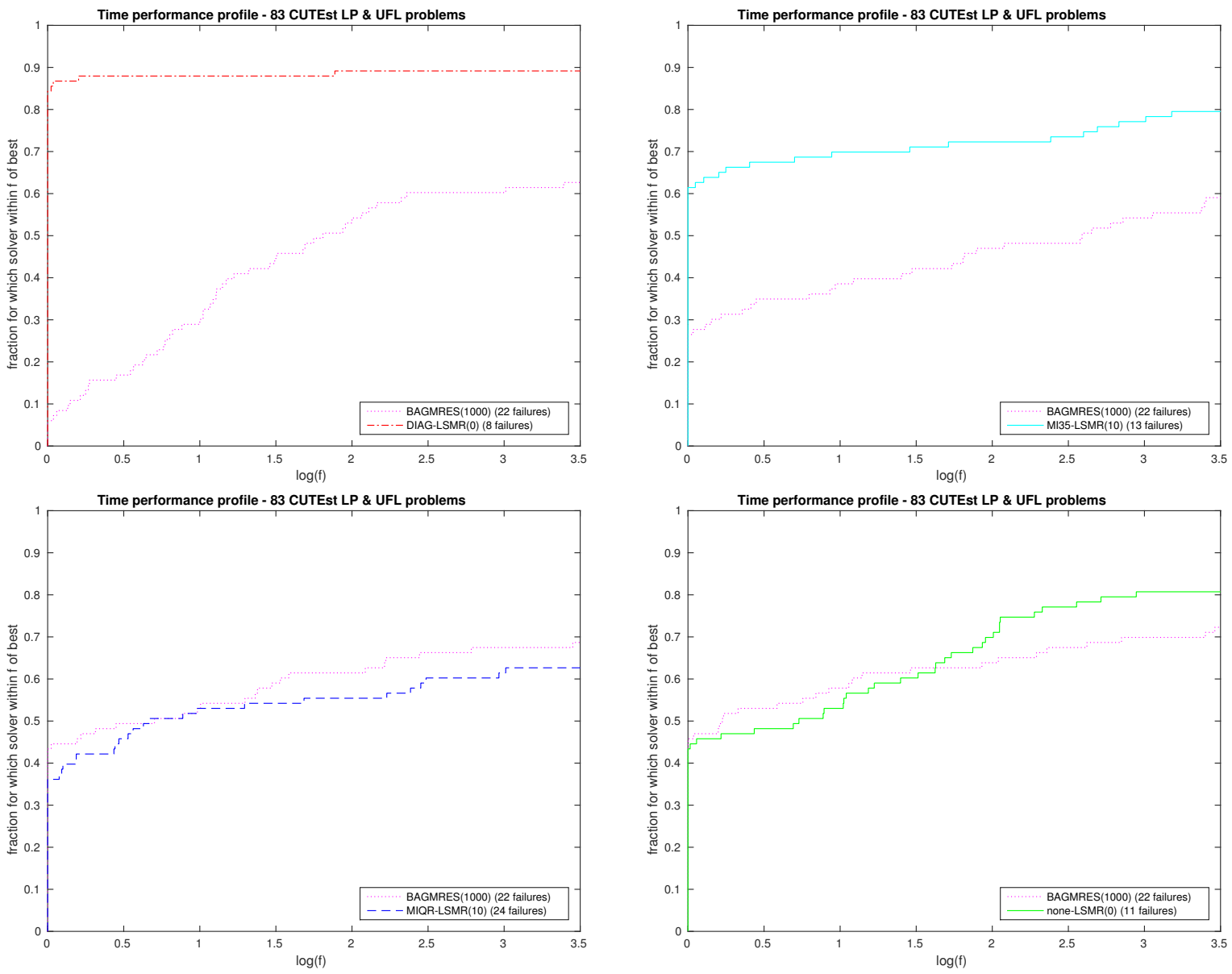

Fig. 14. Time performance profile for BA-GMRES(1000) and LSMR with diagonal, IC (HSL_MI35), MIQR and no preconditioning for test set $\mathcal{T}$.

the time limit or iteration count limit has been reached without satisfying (6), C1 or $\mathrm{C} 2$ is actually satisfied and for other examples, a larger value of $\delta_{3}$ would still have resulted in $\mathrm{C} 1$ or $\mathrm{C} 2$ holding (and thus our reported iteration counts and total times can sometimes be larger than necessary). However, for some problems, including the TFxx examples, a smaller $\delta_{3}$ is needed to satisfy C1 or C2. For example, for MINRES with $\delta_{3}=10^{-11}, \mathrm{C} 1$ is satisfied for problems TF14 and TF15 (the iteration counts increase from 1987 and 1107 to 10,700 and 46,341, respectively, which are similar to those needed by LSMR with HSL_MI35). But for the other TFxx problems, the number of iterations needed to satisfy C1 exceeds our limit of 100,000 . Note that we were unable to solve problem IMDB to the required accuracy (with our time and iteration count limits) using any of the direct solvers or preconditioners in this study, while problems NotreDame_actors, TF17, TF18, TF19 and wheel_601 proved impossible to all but a few solvers. 


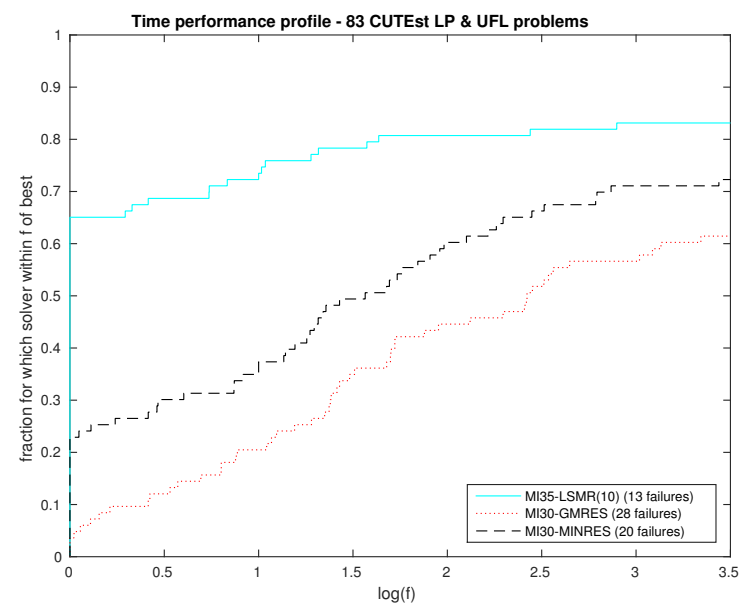

Fig. 15. Time performance profile for LSMR with IC (HSL_MI35) preconditioning and GMRES(1000) and MINRES with signed IC (HSL_MI30) preconditioning for test set $\mathcal{T}$.

\subsection{Performance comparison with a direct solver}

In Figure 16, we present time performance profiles for the direct solver HSL_MA87, applied to the (prescaled and slightly shifted) normal equations (4), and for diagonal and IC (HSL_MI35) preconditioned LSMR. We see that for the set $\mathcal{T}$ the direct solver is the fastest for almost $70 \%$ of the problems, but it is unable to solve $18 \%$ of the problems for which there was insufficient memory. If we look at the results for individual problems given in Table VI, we see that both diagonal and IC preconditioned LSMR solve some problems that HSL_MA87 fail on, including SPAL_004 and the GL7dxx examples.
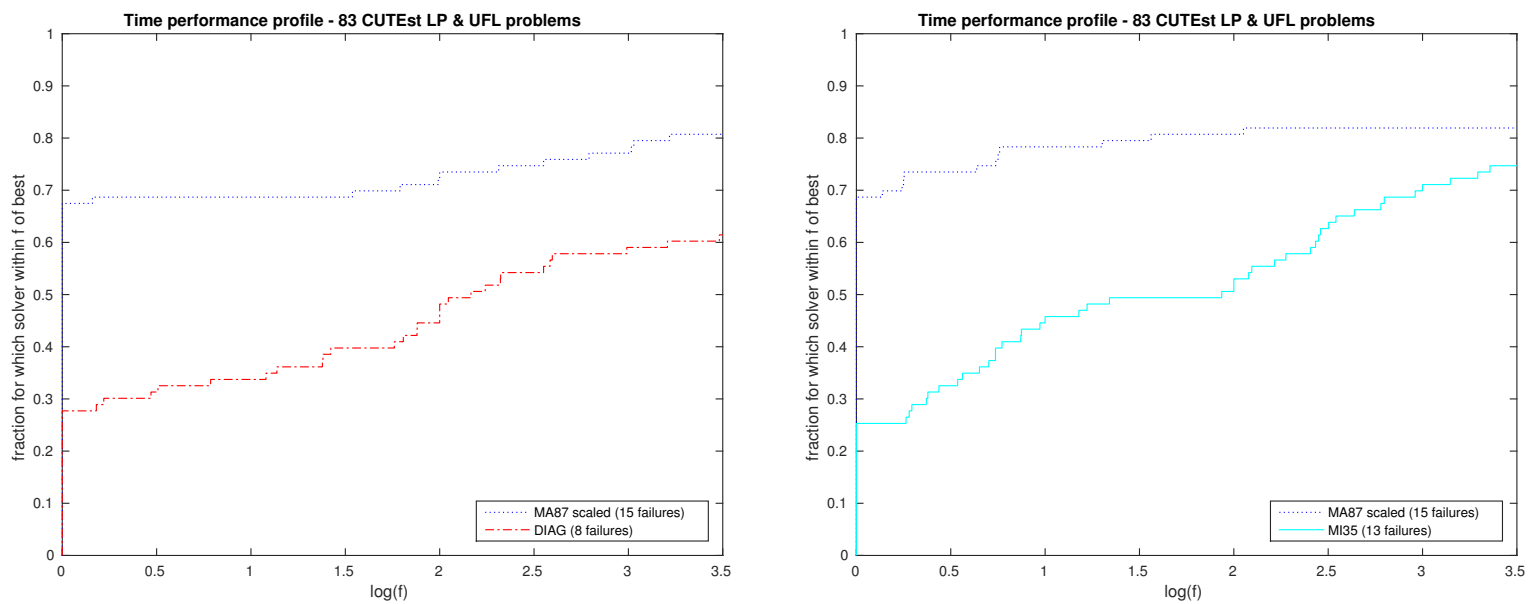

Fig. 16. Time performance profile for direct solver HSL_MA87 and diagonal and IC (HSL_MI35) preconditioned LSMR for test set $\mathcal{T}$. 


\subsection{Summary tables}

In Tables II-VI, we present summary data that allows a direct comparison of a particular statistic across the range of methods considered. We remove SPQR and HSL_MA97 (for the augmented system) as these perform less well than HSL_MA87 (for the normal equations). Similarly, MINRES preconditioned by HSL_MI30 is included (denoted by MI30-MIN) while GMRES preconditioned by HSL_MI30 is omitted. Full results for all methods (including those omitted here) may be found in [30]. For the iterative methods, we have selected what appears to be the "best" global choice of localsize or gmres_its as appropriate; these are localsize=0 for the unpreconditioned and diagonal LSMR, localsize=10 for the MIQR, RIF, and HSL_MI35 versions, and gmres_its=1000 for BA-GMRES (denoted in the tables by BA-G). We summarise the storage required for the factors (and for GMRES), the number of iterations performed, the elapsed time required to build the preconditioner and the total elapsed time to solve the problem (using 4 processors) and report the computed least squares residual. Note that, in Table III, the iteration count for BA-GMRES is the number of GMRES iterations whereas for the other methods it is the LSMR iteration count; the direct solvers are not included in this table since the iteration count is always 1. Similarly, we omit columns for the space and times required to obtain factors when no preconditioning is used in Tables II and IV, respectively, as well as the factorization times for BA-GMRES from the later, as the computation of this preconditioner is integrated within the overall algorithm. A - indicates that the run was unsuccessful; again, for full details the reader is referred to [30].

\section{CONCLUDING REMARKS}

In this study, we have compared the performances of a number of preconditioning techniques for sparse linear least-squares problems. Our main tool has been performance profiles, but the complete numerical results are also available [30]. The findings of our study confirm that preconditioning least-squares problems is hard and that at present there is no single approach that works well for all problems; we thus conclude that there is scope for considerable further developments in this area. We have found that, in many cases, diagonal preconditioning performs as well as or better than more sophisticated approaches and, as it is very simple to implement and to apply (and can be used in parallel), we would suggest trying diagonal preconditioning first. Investigating extending simple diagonal preconditioning to a block diagonal approach (combined with a preordering step) would be interesting (note that block diagonal preconditioning is currently offered by the Ceres non-linear least-squares solver [1]). In terms of iteration counts, using an incomplete factorization of the normal equations performs well and, as we would expect since diagonal preconditioning can be regarded as a special case in which only one entry per row/column is retained, it generally requires far fewer iterations than diagonal preconditioning.

We observe that the direct solvers and the incomplete factorization codes HSL_MI30 and HSL_MI35 include options for scaling (and use scaling by default) whereas the software for MIQR, RIF and BA-GMRES that is currently available does not offer scaling. It would be of interest in the future to examine how much the performance of these approaches can be improved by the incorporation of scaling.

A further contribution of this study has been a detailed comparison of the LSQR and LSMR methods and of the effect of local reorthogonalization within LSMR. Our findings have confirmed those of Fong and Saunders [24] and have shown that the choice of the best local reorthogonalization parameter is problem and preconditioner dependent and also depends on whether reducing the iteration count or the total time is the primary objective. 
Finally, we observe that a number of other approaches have been proposed in recent years, including the limited memory preconditioner (LMP) of Bellavia, Gonzio and Morini [6] and the balanced incomplete factorization (BIF) preconditioner of Bru, Marín Mas and Tůma [12]. LU preconditioning, which was discussed by Saunders [62] in 1979 (see also Section 7.5.3 of the book by Björck [10]), has also received renewed attention (see the 2015 paper by Arioli and Duff [3] and presentation by Saunders [64]). These are not included in this study since implementations that allow timings that are suitable for making fair comparisons with our software are not currently available and the algorithms are sufficiently complicated for it to be infeasible for us to develop efficient implementations for use here. Note that in [3] and [6], experimental results are reported using MATLAB codes. Unfortunately, the recent Fortran results reported by Saunders [64] do not encourage us to expect that the LU approach will be efficient in terms of time. But it would be interesting to see if it can be used to solve some of the examples that are currently intractable. In particular, we recommend that future comparisons of linear least-squares software includes the test examples PDE1, IMDB, GLRD17-21, NotreDame_actors, TF17-19 and wheel_601, since these challenge many of the methods we have considered here.

\section{Acknowledgements}

We are grateful to Michael Saunders for a number of discussions related to his LSQR and LSMR software packages and for making our reverse communication implementation of LSMR available on his web page. We thank Miroslav Tủma for help with understanding and employing his RIF code. We would also like to thank four anonymous reviewers for their constructive feedback.

\section{REFERENCES}

S. Agarwal, K. Mierle, AND Others, Ceres solver. http://ceres-solver.org.

P. Amestoy, I. S. DUfF, AND C. PUGlisi, Multifrontal QR factorization in a multiprocessor environment, Numerical Linear Algebra with Applications, 3 (1996), pp. 275-300.

M. ARIOLI AND I. S. DUFF, Preconditioning linear least-squares problems by identifying a basis matrix, SIAM J. on Scientific Computing, 37 (2015), pp. S544-S561.

Z.-Z. BAI, I. S. DUFF, AND A. J. WATHEN, A class of incomplete orthogonal factorization methods. I: Methods and theories, BIT Numerical Mathematics, 41 (2001), pp. 53-70.

Z.-Z. BAI, I. S. DUFF, AND J.-F. YIN, Numerical study on incomplete orthogonal factorization preconditioners, J. of Computational and Applied Mathematics, 226 (2009), pp. 22-41.

S. Bellavia, J. Gondzio, AND B. MoRIni, A matrix-free preconditioner for sparse symmetric positive definite systems and least-squares problems, SIAM J. on Scientific Computing, 35 (2013), pp. A192A211.

M. BEnZI, Preconditioning techniques for large linear systems: a survey, J. of Computational Physics, 182 (2002), pp. 418-477.

M. BENZI AND M. TŮmA, A robust incomplete factorization preconditioner for positive definite matrices, Numerical Linear Algebra with Applications, 10 (2003), pp. 385-400.

, A robust preconditioner with low memory requirements for large sparse least squares problems, SIAM J. on Scientific Computing, 25 (2003), pp. 499-512.

Å. BJÖRCK, Numerical methods for Least Squares Problems, SIAM, Philadelphia, 1996.

Å. Björck, T. Elfving, AND Z. STrakos, Stability of conjugate gradient and Lanczos methods for linear least squares problems, SIAM J. on Matrix Analysis and Applications, 19 (1998), pp. 720-736.

R. BRU, J. MARÍn, J. MAS, AND M. TŮMA, Preconditioned iterative methods for solving linear least squares problems, SIAM J. Sci. Comput., 36 (2014), pp. A2002-A2022.

A. BUTTARI, Fine-grained multithreading for the multifrontal $Q R$ factorization of sparse matrices, SIAM J. on Scientific Computing, 35 (2013), pp. C323-C345.

Y. Chen, T. A. DAvis, W. H. HAGER, AND S. RAJAmAniCKAM, Algorithm 887: CHOLMOD, supernodal sparse Cholesky factorization and update/downdate, ACM Transactions on Mathematical Software, 35 (2008), pp. 22:1-22:14. 
E. CHOW AND Y. SAAD, Experimental study of ILU preconditioners for indefinite matrices, J. of Computational and Applied Mathematics, 86 (1997), pp. 387-414.

G. Cimmino, Calcolo approssimato per le soluzioni dei sistemi di equazioni lineari, Ric. Sci. Progr. tecn. econom. naz., 9 (1939), pp. 326-333.

T. A. DAVIS, Algorithm 915: SuiteSparseQR: Multifrontal multithreaded rank-revealing sparse $Q R$ factorization, ACM Transactions on Mathematical Software, 38 (2011), pp. 8:1-8:22.

T. A. DAVIS AND Y. HU, The University of Florida sparse matrix collection, ACM Transactions on Mathematical Software, 38 (2011), pp. 1:1-1:25.

E. D. DOLAN AND J. J. MORÉ, Benchmarking optimization software with performance profiles, Mathematical Programming, 91 (2002), pp. 201-213.

I. S. DUFF, MA57- a new code for the solution of sparse symmetric definite and indefinite systems, ACM Transactions on Mathematical Software, 30 (2004), pp. 118-154.

I. S. DUFF AND J. Koster, The design and use of algorithms for permuting large entries to the diagonal of sparse matrices, SIAM J. on Matrix Analysis and Applications, 20 (1999), pp. 889-901.

- On algorithms for permuting large entries to the diagonal of a sparse matrix, SIAM J. on Matrix Analysis and Applications, 22 (2001), pp. 973-996.

R. W. FAREBROTHER, Fitting Linear Relationships: A History of the Calculus of Observations 1750-1900, Springer, New York, 1999.

D. C.-L. FONG AND M. A. SAUNDERS, LSMR: An iterative algorithm for sparse least-squares problems, SIAM J. on Scientific Computing, 33 (2011), pp. 2950-2971.

N. I. M. Gould, Y. HU, AND J. A. ScotT, A numerical evaluation of sparse direct symmetric solvers for the solution of large sparse, symmetric linear systems of equations., ACM Transactions on Mathematical Software, 33 (2007). Article 10, 32 pages.

N. I. M. Gould, D. ORBAN, AND P. L. Toint, CUTEst: a constrained and unconstrained testing environment with safe threads for mathematical optimization, Computational Optimization and Applications, 60 (2015), pp. 545-557.

N. I. M. Gould, D. ORBAN, AND PH. L. ToInT, GALAHAD—a library of thread-safe fortran 90 packages for large-scale nonlinear optimization, ACM Transactions on Mathematical Software, 29 (2003), pp. 353372.

N. I. M. Gould AND J. A. ScotT, A numerical evaluation of HSL packages for the direct solution of large sparse, symmetric linear systems of equations, ACM Transactions on Mathematical Software, 30 (2004), pp. 300-325.

N. I. M. Gould AND J. A. ScotT, A note on performance profiles for benchmarking software, Technical Report RAL-P-2015-004, Rutherford Appleton Laboratory, 2015.

, The state-of-the-art of preconditioners for sparse linear least squares problems: the complete results, Technical Report RAL-TR-2015-009 (revision 1), Rutherford Appleton Laboratory, 2016.

J. F. GRCAR, M. A. SAUNDERS, AND Z. SU, Estimates of optimal backward perturbations for linear least squares problems, Technical Report SOL 2007-1, Department of Management Science and Engineering, Stanford University, Stanford, 2007.

C. GREIF, S. HE, AND P. LIU, SYM-ILDL: incomplete LDLT factorization of symmetric indefinite and skew symmetric matrices, technical report, Department of Computer Science, The University of British Columbia, 2015. Software available from http://www.cs.ubc.ca/ inutard/html/.

A. GUPTA, WSMP Watson sparse matrix package (Part II): direct solution of general sparse systems, Technical Report RC 21888 (98472), IBM T.J. Watson Research Center, 2000.

K. HAYAMI, J.-F. YIN, AND T. ITO, GMRES methods for least squares problems, SIAM J. on Matrix Analysis and Applications, 31 (2010), pp. 2400-2430.

M. R. Hestenes AND E. STIEFEL, Methods of conjugate gradients for solving linear systems, J. of Research of the National Bureau of Standards, 49 (1952), pp. 409-435.

J. D. HogG, E. Ovtchinnikov, AND J. A. ScotT, A sparse symmetric indefinite direct solver for GPU architectures, Preprint RAL-P-2014-006, Rutherford Appleton Laboratory, 2014.

J. D. HoGG, J. K. REID, AND J. A. ScotT, Design of a multicore sparse Cholesky factorization using DAGs, SIAM J. on Scientific Computing, 32 (2010), pp. 3627-3649.

J. D. HogG AND J. A. ScotT, HSL_MA97: a bit-compatible multifrontal code for sparse symmetric systems, Technical Report RAL-TR-2011-024, Rutherford Appleton Laboratory, 2011.

, New parallel sparse direct solvers for multicore architectures, Algorithms, 6 (2013), pp. 702-725. Special issue: Algorithms for Multi Core Parallel Computation.

HSL. A collection of Fortran codes for large-scale scientific computation, 2016. http://www.hsl.rl.ac.uk. 
A. Jennings And M. A. AJIZ, Incomplete methods for solving $A^{T} A x=b$, SIAM J. on Scientific and Statistical Computing, 5 (1984), pp. 978-987.

P. JiRAneK AND D. TITLEY-PeloqUin, Estimating the backward error in LSQR, SIAM J. on Matrix Analysis and Applications, 31 (2010), pp. 2055-2074.

S. KACZmarZ, Ängenäherte Auflösung von Systemen linearer Gleichungen, Bull. Internat. Acad. Polon. Sci. Cl. A., (1937), pp. 355-356.

I. E. KAPORIN, High quality preconditioning of a general symmetric positive definite matrix based on its $U^{T} U+U^{T} R+R^{T} U$ decomposition, Numerical Linear Algebra with Applications, 5 (1998), pp. 483509 .

N. LI AND Y. SAAD, Crout versions of ILU factorization with pivoting for sparse symmetric matrices, Electronic Transactions on Numerical Analysis, 20 (2005), pp. 75-85.

- MIQR: A multilevel incomplete $Q R$ preconditioner for large sparse least-squares problems, SIAM J. on Matrix Analysis and Applications, 28 (2006).

T. A. MANTEUfFel, An incomplete factorization technique for positive definite linear systems, Mathematics of Computation, 34 (1980), pp. 473-497.

K. MORIKUNI AND K. HAYAMI, Inner-iteration Krylov subspace methods for least squares problems, SIAM J. on Matrix Analysis and Applications, 34 (2013), pp. 1-22.

- Convergence of inner-iteration GMRES methods for rank deficient least squares problems, SIAM J. on Matrix Analysis and Applications, 36 (2015), pp. 225-250.

MUMPS 5.0.0: a multifrontal massively parallel sparse direct solver, 2015. http://mumps-solver.org.

A. R. L. Oliveira AND D. C. Sorensen, A new class of preconditioners for large-scale linear systems from interior point methods for linear programming, Linear Algebra and its Applications, 394 (2005), pp. 1-24.

C. C. PAige AND M. A. SAUnders, Solution of sparse indefinite systems of linear equations, SIAM J. on Numerical Analysis, 12 (1975), pp. 617-629.

- Algorithm 583; LSQR: Sparse linear equations and least-squares problems, ACM Transactions on Mathematical Software, 8 (1982), pp. 195-209.

_ LSQR: An algorithm for sparse linear equations and sparse least squares, ACM Transactions on Mathematical Software, 8 (1982), pp. 43-71.

A. T. Papadopoulus, I. S. DUFF, AND A. J. Wathen, A class of incomplete orthogonal factorization methods. II: Implementation and results, BIT Numerical Mathematics, 45 (2005), pp. 159-179.

PARDISO 5.0.0 solver project, 2014. http://www.pardiso-project.org.

J. K. REID AND J. A. ScotT, An out-of-core sparse Cholesky solver., ACM Transactions on Mathematical Software, 36 (2009). Article 9, 33 pages.

D. RUIZ, A scaling algorithm to equilibrate both rows and columns norms in matrices, Technical Report RAL-TR-2001-034, Rutherford Appleton Laboratory, Chilton, Oxfordshire, England, 2001.

Y. SAAD, Preconditioning techniques for nonsymmetric and indefinite linear systems, J. of Computational and Applied Mathematics, 24 (1988), pp. 89-105.

- Iterative Methods for Sparse Linear Systems, Society for Industrial and Applied Mathematics, Philadelphia, PA, second ed., 2003.

Y. SAAD AND M. H. SCHULZ, GMRES: A generalized minimal residual algorithm for solving nonsymmetric linear systems, SIAM Journal on Scientific and Statistical Computing, 7 (1986), pp. 856-869.

M. A. SAUNDERS, Sparse least squares problems by conjugate gradients: a comparison of preconditioning methods, in Proceedings of Computer Science and Statistics: Twelfth Annual Conference on the Interface, Waterloo, Canada, 1979.

, Cholesky-based methods for sparse least squares: The benefits of regularization, in Linear and Nonlinear Conjugate Gradient-Related Methods, L. Adams and J. L. Nazareth, eds., Philadelphia, USA, 1996, SIAM, pp. 92-100.

,$- L U$ preconditioning for full rank and singular sparse least squares, 2015. Presentation at SIAM Conference on Applied Linear Algebra (LA15) available from https://www.pathlms.com/siam/courses/ 1697/sections/2326.

J. A. Sсотт, On using Cholesky-based factorizations for solving rank-deficient sparse linear least-squares problems, Technical Report RAL-P-2016-006, Rutherford Appleton Laboratory, 2016.

J. A. ScOTT AND M. TŮMA, HSL_MI28: an efficient and robust limited-memory incomplete Cholesky factorization code, ACM Transactions on Mathematical Software, 40 (2014), pp. Art. 24, 19.

, On positive semidefinite modification schemes for incomplete Cholesky factorization, SIAM J. on Scientific Computing, 36 (2014), pp. A609-A633. 
Preconditioners for sparse linear least-squares problems

, On signed incomplete Cholesky factorization preconditioners for saddle-point systems, SIAM J. on Scientific Computing, 36 (2014), pp. A2984-A3010.

, Solving symmetric indefinite systems using memory efficient incomplete factorization preconditioners, Technical Report RAL-P-2015-002, Rutherford Appleton Laboratory, 2015.

, Preconditioning of linear least squares by RIF for implicitly held normal equations, Technical Report RAL-TR-2016-P-001, Rutherford Appleton Laboratory, 2016.

M. Tismenetsky, A new preconditioning technique for solving large sparse linear systems, Linear Algebra and its Applications, 154-156 (1991), pp. 331-353.

X. WANG, K. A. GAllivan, AND R. BRAMley, CIMGS: an incomplete orthogonal factorization preconditioner, SIAM J. on Scientific Computing, 18 (1997), pp. 516-536. 


\section{Appendix}

For each problem in the test subset $\mathcal{T}$ described in Section 2.1, $m, n$ and $n z(A)$ are the row and column counts and the number of nonzeros in $A$. In addition, "nullity" is the estimated deficiency in the rank as computed by HSL_MA97, "density(A)" is the largest ratio of number of nonzeros in a row to $n$ over all rows, and "density(C)" is the ratio of the number of entries in $C$ to $n^{2}$. A * indicates a right-hand side vector $b$ was supplied and - denotes insufficient memory to compute the statistic.

Table I: Statistics for the Test Set $\mathcal{T}$.

\begin{tabular}{|c|c|c|c|c|c|c|}
\hline name & $m$ & $n$ & $n z(A)$ & nullity & $\operatorname{density}(\mathrm{A})$ & density(C) \\
\hline \multicolumn{7}{|c|}{ CUTEst examples } \\
\hline BAS1LP & 9825 & 5411 & 587775 & 0 & $6.75 \mathrm{E}-2$ & $8.87 \mathrm{E}-2$ \\
\hline BAXTER & 30733 & 27441 & 111576 & 2993 & $1.68 \mathrm{E}-3$ & $1.63 \mathrm{E}-3$ \\
\hline BCDOUT & 7078 & 5412 & 67344 & 2 & $1.55 \mathrm{E}-1$ & $6.86 \mathrm{E}-2$ \\
\hline $\mathrm{CO9}$ & 22924 & 10789 & 109651 & 0 & $2.60 \mathrm{E}-3$ & $2.14 \mathrm{E}-3$ \\
\hline CONT11_L & 1961394 & 1468599 & 5382999 & 0 & $4.77 \mathrm{E}-6$ & $8.38 \mathrm{E}-6$ \\
\hline DBIR1 & 45775 & 18804 & 1077025 & 103 & $1.19 \mathrm{E}-2$ & $6.89 \mathrm{E}-3$ \\
\hline DBIR2 & 45877 & 18906 & 1158159 & 101 & $1.23 \mathrm{E}-2$ & $7.38 \mathrm{E}-3$ \\
\hline D2Q06C & 5831 & 2171 & 33081 & 0 & $1.57 \mathrm{E}-2$ & $1.19 \mathrm{E}-2$ \\
\hline DELF000 & 5543 & 3128 & 13741 & 0 & $2.88 \mathrm{E}-3$ & $2.74 \mathrm{E}-3$ \\
\hline GE & 16369 & 10099 & 44825 & 0 & $3.56 \mathrm{E}-3$ & $1.10 \mathrm{E}-3$ \\
\hline LARGE001 & 7176 & 4162 & 18887 & 0 & $2.64 \mathrm{E}-3$ & $2.46 \mathrm{E}-3$ \\
\hline LPL1 & 129959 & 39951 & 386218 & 44 & $4.00 \mathrm{E}-4$ & $3.39 \mathrm{E}-4$ \\
\hline MOD2 & 66409 & 34774 & 199810 & 0 & $4.60 \mathrm{E}-4$ & $5.00 \mathrm{E}-4$ \\
\hline MODEL10 & 16819 & 4400 & 150372 & 0 & $3.86 \mathrm{E}-3$ & $1.51 \mathrm{E}-2$ \\
\hline MPSBCD 03 & 7078 & 5412 & 66210 & 2 & $1.55 \mathrm{E}-1$ & $6.82 \mathrm{E}-2$ \\
\hline NSCT2 & 37563 & 23003 & 697738 & 287 & $2.73 \mathrm{E}-2$ & $1.57 \mathrm{E}-2$ \\
\hline NSIR2 & 10057 & 4453 & 154939 & 0 & $5.28 \mathrm{E}-2$ & $2.39 \mathrm{E}-2$ \\
\hline PDE1 & 271792 & 270595 & 990587 & - & $6.70 \mathrm{E}-1$ & \\
\hline PDS-100 & 514577 & 156016 & 1096002 & 227 & $1.92 \mathrm{E}-5$ & $6.04 \mathrm{E}-5$ \\
\hline PDS-90 & 475448 & 142596 & 1014136 & 227 & $2.10 \mathrm{E}-5$ & $6.71 \mathrm{E}-5$ \\
\hline PILOT-JA & 2267 & 940 & 14977 & 0 & $5.85 \mathrm{E}-2$ & $3.36 \mathrm{E}-2$ \\
\hline PILOTNOV & 2446 & 975 & 13331 & 0 & $4.10 \mathrm{E}-2$ & $2.65 \mathrm{E}-2$ \\
\hline RAIL2586 & 923269 & 2586 & 8011362 & 0 & $4.64 \mathrm{E}-3$ & $7.05 \mathrm{E}-2$ \\
\hline RAIL4284 & 1096894 & 4284 & 11284032 & 0 & $2.80 \mathrm{E}-3$ & $1.19 \mathrm{E}-1$ \\
\hline SPAL_004 & 321696 & 10203 & 46168124 & 0 & $1.65 \mathrm{E}-2$ & $4.99 \mathrm{E}-1$ \\
\hline STAT96V2 & 957432 & 29089 & 2852184 & 0 & $4.13 \mathrm{E}-4$ & $4.17 \mathrm{E}-4$ \\
\hline STAT96V3 & 1113780 & 33841 & 3317736 & 0 & $3.55 \mathrm{E}-4$ & $3.58 \mathrm{E}-4$ \\
\hline STAT96V4 & 63076 & 3173 & 491336 & 0 & $2.84 \mathrm{E}-3$ & $5.43 \mathrm{E}-3$ \\
\hline STORMG21K & 1377306 & 526185 & 3459881 & 0 & $1.93 \mathrm{E}-3$ & $3.00 \mathrm{E}-4$ \\
\hline WATSON_1 & 386992 & 201155 & 1055093 & 0 & $4.47 \mathrm{E}-5$ & $4.79 \mathrm{E}-5$ \\
\hline WATSON_2 & 677224 & 352013 & 1846391 & 0 & $4.26 \mathrm{E}-5$ & $2.74 \mathrm{E}-5$ \\
\hline WORLD & 67147 & 34506 & 198883 & 0 & $4.64 \mathrm{E}-4$ & $4.89 \mathrm{E}-4$ \\
\hline \multicolumn{7}{|c|}{ UF Sparse Matrix Collection examples } \\
\hline 12month1 & 872622 & 12471 & 22624727 & & $2.74 \mathrm{E}-1$ & $6.87 \mathrm{E}-1$ \\
\hline 162bit & 3606 & 3476 & 37118 & 16 & $4.03 \mathrm{E}-3$ & $1.95 \mathrm{E}-2$ \\
\hline 176bit & 7441 & 7150 & 82270 & 40 & $2.24 \mathrm{E}-3$ & $1.03 \mathrm{E}-2$ \\
\hline 192bit & 13691 & 13093 & 154303 & 82 & $1.22 \mathrm{E}-3$ & $5.73 \mathrm{E}-3$ \\
\hline 208bit & 24430 & 23191 & 299756 & 199 & $7.76 \mathrm{E}-4$ & $3.56 \mathrm{E}-3$ \\
\hline beaflw & 500 & 492 & 53403 & 4 & $8.13 \mathrm{E}-1$ & 8.94E-1 \\
\hline c8_mat11 & 5761 & 4562 & 2462970 & 0 & $5.30 \mathrm{E}-1$ & $8.12 \mathrm{E}-1$ \\
\hline
\end{tabular}


Table I: Statistics for the Test Set $\mathcal{T}$ (continued).

\begin{tabular}{|c|c|c|c|c|c|c|}
\hline name & $m$ & $n$ & $n z(A)$ & nullity & $\operatorname{density}(\mathrm{A})$ & $\operatorname{density}(\mathrm{C})$ \\
\hline connectus & 394707 & 458 & 1127525 & 0 & $1.59 \mathrm{E}-1$ & $1.58 \mathrm{E}-1$ \\
\hline ESOC & 327062 & 37349 & 6019939 & 0 & $5.09 \mathrm{E}-4$ & $5.16 \mathrm{E}-3$ \\
\hline EternityII_Etilde & 204304 & 10054 & 1170516 & 0 & $6.96 \mathrm{E}-4$ & $1.70 \mathrm{E}-2$ \\
\hline f855_mat9 & 2511 & 2456 & 171214 & 0 & $3.38 \mathrm{E}-1$ & $7.44 \mathrm{E}-1$ \\
\hline GL7d16 & 955127 & 460260 & 14488881 & - & $1.39 \mathrm{E}-4$ & $9.46 \mathrm{E}-4$ \\
\hline GL7d17 & 1548649 & 955127 & 25978098 & - & $7.22 \mathrm{E}-5$ & $4.43 \mathrm{E}-4$ \\
\hline GL7d18 & 1955309 & 1548645 & 35590540 & - & $4.71 \mathrm{E}-5$ & $2.54 \mathrm{E}-4$ \\
\hline GL7d19 & 1955296 & 1911130 & 37322725 & - & $2.83 \mathrm{E}-5$ & $1.97 \mathrm{E}-4$ \\
\hline GL7d20 & 1911124 & 1437546 & 29893084 & - & $2.99 \mathrm{E}-5$ & $2.23 \mathrm{E}-4$ \\
\hline GL7d21 & 1437546 & 822922 & 18174775 & - & $4.37 \mathrm{E}-5$ & $3.26 \mathrm{E}-4$ \\
\hline GL7d22 & 822906 & 349443 & 8251000 & - & $7.44 \mathrm{E}-5$ & $6.28 \mathrm{E}-4$ \\
\hline GL7d23 & 349443 & 105054 & 2695430 & - & $1.81 \mathrm{E}-4$ & $1.65 \mathrm{E}-3$ \\
\hline graphics & 29493 & 11822 & 117954 & 0 & $3.38 \mathrm{E}-4$ & $5.91 \mathrm{E}-4$ \\
\hline HFE18_96_in & 2372 & 2371 & 933343 & 0 & $5.07 \mathrm{E}-1$ & $9.91 \mathrm{E}-1$ \\
\hline IG5-15 & 11369 & 6146 & 323509 & 0 & $1.95 \mathrm{E}-2$ & $1.52 \mathrm{E}-1$ \\
\hline IG5-16 & 18846 & 9519 & 588326 & 0 & $1.26 \mathrm{E}-2$ & $1.28 \mathrm{E}-1$ \\
\hline IG5-17 & 30162 & 14060 & 1035008 & 0 & $8.53 \mathrm{E}-3$ & $1.14 \mathrm{E}-1$ \\
\hline IG5-18 & 47894 & 20818 & 1790490 & 0 & $5.76 \mathrm{E}-3$ & $9.91 \mathrm{E}-2$ \\
\hline IMDB & 896302 & 303617 & 3782463 & - & $5.24 \mathrm{E}-3$ & $1.51 \mathrm{E}-3$ \\
\hline kneser_10_4_1 & 349651 & 330751 & 992252 & - & $4.84 \mathrm{E}-5$ & $8.89 \mathrm{E}-5$ \\
\hline landmark & 71952 & 2673 & 1146848 & 2 & $5.99 \mathrm{E}-3$ & $1.68 \mathrm{E}-2$ \\
\hline LargeRegFile & 2111154 & 801374 & 4944201 & 0 & $4.99 \mathrm{E}-6$ & $9.93 \mathrm{E}-6$ \\
\hline Maragal_6* & 21251 & 10144 & 537694 & 516 & $5.86 \mathrm{E}-1$ & $7.49 \mathrm{E}-1$ \\
\hline Maragal_7* & 46845 & 26525 & 1200537 & 2046 & $3.60 \mathrm{E}-1$ & $3.10 \mathrm{E}-1$ \\
\hline Maragal_8* & 60845 & 33093 & 1308415 & 7107 & $5.03 \mathrm{E}-2$ & $3.56 \mathrm{E}-2$ \\
\hline mri1 & 114637 & 65536 & 589824 & 603 & $3.66 \mathrm{E}-3$ & $2.57 \mathrm{E}-4$ \\
\hline mri2 & 104597 & 63240 & 569160 & - & $6.60 \mathrm{E}-2$ & $7.84 \mathrm{E}-3$ \\
\hline NotreDame_actors & 383640 & 127823 & 1470404 & - & $5.05 \mathrm{E}-3$ & $2.52 \mathrm{E}-3$ \\
\hline psse $0^{*}$ & 26722 & 11028 & 102432 & 0 & $3.63 \mathrm{E}-4$ & $5.88 \mathrm{E}-4$ \\
\hline psse1* & 14318 & 11028 & 57376 & 0 & $1.63 \mathrm{E}-3$ & $6.67 \mathrm{E}-4$ \\
\hline psse2* & 28634 & 11028 & 115262 & 0 & $2.54 \mathrm{E}-3$ & $7.68 \mathrm{E}-4$ \\
\hline rel9 & 5921786 & 274667 & 23667183 & - & $1.46 \mathrm{E}-5$ & $5.09 \mathrm{E}-4$ \\
\hline relat9 & 9746232 & 274667 & 38955420 & - & $1.46 \mathrm{E}-5$ & $5.09 \mathrm{E}-4$ \\
\hline Rucci1 & 1977885 & 109900 & 7791168 & 0 & $3.64 \mathrm{E}-5$ & $8.07 \mathrm{E}-4$ \\
\hline sls & 1748122 & 62729 & 6804304 & 0 & $6.38 \mathrm{E}-5$ & $1.20 \mathrm{E}-3$ \\
\hline TF14 & 3159 & 2644 & 29862 & 0 & $4.92 \mathrm{E}-3$ & $3.12 \mathrm{E}-2$ \\
\hline TF15 & 7741 & 6334 & 80057 & 0 & $2.21 \mathrm{E}-3$ & $1.63 \mathrm{E}-2$ \\
\hline TF16 & 19320 & 15437 & 216173 & 0 & $9.72 \mathrm{E}-4$ & $8.17 \mathrm{E}-3$ \\
\hline TF17 & 48629 & 38132 & 586218 & - & $4.20 \mathrm{E}-4$ & $3.98 \mathrm{E}-3$ \\
\hline TF18 & 123867 & 95368 & 1597545 & - & $1.78 \mathrm{E}-4$ & $1.88 \mathrm{E}-3$ \\
\hline TF19 & 317955 & 241029 & 4370721 & - & $7.47 \mathrm{E}-5$ & $8.70 \mathrm{E}-4$ \\
\hline tomographic1* & 59360 & 45908 & 647495 & 3436 & $3.27 \mathrm{E}-4$ & $8.68 \mathrm{E}-4$ \\
\hline Trec14 & 15904 & 3159 & 2872265 & 0 & $7.91 \mathrm{E}-1$ & $9.32 \mathrm{E}-1$ \\
\hline wheel_601 & 902103 & 723605 & 2170814 & - & $8.32 \mathrm{E}-4$ & $4.22 \mathrm{E}-4$ \\
\hline
\end{tabular}


Table II: Storage Required for Factors (or for GMRES) for Subset CUTEst Problems by Each Method.

\begin{tabular}{|c|c|c|c|c|c|c|c|c|c|c|}
\hline name & $m$ & $n$ & $n z(A)$ & diagonal & MIQR & RIF & BA-G & MI35 & MI30-MIN & MA87 \\
\hline BAS1LP & 9825 & 5411 & 587775 & 5411 & 154335 & 58422 & 6419411 & 113414 & 858406 & 2525076 \\
\hline BAXTER & 30733 & 27441 & 111576 & 27441 & 166672 & 254733 & 27441 & 218513 & 709648 & 6763027 \\
\hline BCDOUT & 7078 & 5412 & 67344 & 5412 & 227118 & 38953 & 5412 & 97350 & 214193 & 2155189 \\
\hline $\mathrm{CO9}$ & 22924 & 10789 & 109651 & 10789 & 139168 & 52621 & 11802789 & 122520 & 346055 & 1233194 \\
\hline CONT11_L & 1961394 & 1468599 & 5382999 & 1468599 & $>6823917$ & 4883216 & 149807602 & 10208898 & 22208667 & 128552555 \\
\hline DBIR1 & 45775 & 18804 & 1077025 & 18804 & 306833 & 92947 & 7055662 & 264644 & 1977702 & 4589933 \\
\hline DBIR2 & 45877 & 18906 & 1158159 & 18906 & 311225 & 94898 & 17102778 & 172316 & 1876024 & 4846892 \\
\hline D2Q06C & 5831 & 2171 & 33081 & 2171 & 22404 & 13660 & 700243 & 30258 & 100726 & 203218 \\
\hline DELFO00 & 5543 & 3128 & 13741 & 3128 & 9959 & 11667 & 4134128 & 11314 & 33260 & 136175 \\
\hline $\mathrm{GE}$ & 16369 & 10099 & 44825 & 10099 & 98350 & 49164 & 8719998 & 134483 & 248210 & 710847 \\
\hline LARGE001 & 7176 & 4162 & 18887 & 4162 & 14243 & 16930 & 5169162 & 16000 & 46375 & 204012 \\
\hline LPL1 & 129959 & 39951 & 386218 & 39951 & 184639 & 143744 & 28994143 & 541699 & 1605246 & 7939663 \\
\hline MOD2 & 66409 & 34774 & 199810 & 34774 & 559518 & 229986 & 20505898 & 527770 & 1300573 & 4521661 \\
\hline MODEL10 & 16819 & 4400 & 150372 & 4400 & 34670 & 24083 & 1863308 & 78015 & 378047 & 659462 \\
\hline MPSBCD03 & 7078 & 5412 & 66210 & 5412 & 228815 & 38965 & 5412 & 98584 & 220678 & 2055166 \\
\hline NSCT2 & 37563 & 23003 & 697738 & 23003 & 561767 & 109287 & 4781258 & 165302 & 1407946 & 8399426 \\
\hline NSIR2 & 10057 & 4453 & 154939 & 4453 & 98551 & 27196 & 984313 & 54142 & 305406 & 635304 \\
\hline PDE1 & 271792 & 270595 & 990587 & 270595 & $>7750326$ & $>996368$ & 270595 & & & \\
\hline PDS- 100 & 514577 & 156016 & 1096002 & 156016 & 1027112 & 618440 & 12019236 & 2891511 & 7696832 & 57854930 \\
\hline PDS -90 & 475448 & 142596 & 1014136 & 142596 & 950792 & 570713 & 10557652 & 2626289 & 7233396 & 51441581 \\
\hline PILOT-JA & 2267 & 940 & 14977 & 940 & 9650 & 5780 & 427458 & 10870 & 47073 & 105608 \\
\hline PILOTNOV & 2446 & 975 & 13331 & 975 & 9320 & 5958 & 449095 & 11317 & 44818 & 98127 \\
\hline RAIL2586 & 923269 & 2586 & 8011362 & 2586 & 6235 & 24447 & 495112 & 51833 & 9613272 & 1503568 \\
\hline RAIL4284 & 1096894 & 4284 & 11284032 & 4284 & 6345 & 45055 & 958072 & 89219 & 15880038 & 6776270 \\
\hline SPAL_004 & 321696 & 10203 & 46168124 & 10203 & 18683 & $>6911$ & 20410 & 213972 & 40754070 & 46763011 \\
\hline STAT96V2 2 & 957432 & 29089 & 2852184 & 29089 & 38807 & 81055 & 12573814 & 276290 & 4210897 & 1702787 \\
\hline STAT96V3 & 1113780 & 33841 & 3317736 & 33841 & 45145 & 94273 & 14216653 & 319109 & 4901099 & 1977377 \\
\hline STAT96V4 & 63076 & 3173 & 491336 & 3173 & 11493 & 10513 & 415798 & 47295 & 512268 & 149650 \\
\hline STORMG21K & 1377306 & 526185 & 3459881 & 526185 & 6933837 & $>1850507$ & 526185 & 7505682 & 34433755 & 853778245 \\
\hline WATSON_1 & 386992 & 201155 & 1055093 & 201155 & 1005650 & 519977 & 11469139 & 2634818 & 6106788 & 11698276 \\
\hline WATSON_2 & 677224 & 352013 & 1846391 & 352013 & 2808070 & 1171936 & 17251085 & 4648375 & 10588235 & 17953039 \\
\hline WORLD & 67147 & 34506 & 198883 & 34506 & 535778 & 224464 & 20065186 & 511951 & 1288095 & 4555752 \\
\hline
\end{tabular}


Table II: Storage Required for Factors (or for GMRES) for Subset UF Problems by Each Method.

\begin{tabular}{|c|c|c|c|c|c|c|c|c|c|c|}
\hline name & $m$ & $n$ & $n z(A)$ & diagonal & MIQR & RIF & BA-G & MI35 & MI30-MIN & MA87 \\
\hline 12month1 & 872622 & 12471 & 22624727 & 12471 & 73375 & $>64026$ & 764511 & 260889 & 34096963 & 72985572 \\
\hline $162 \mathrm{bit}$ & 3606 & 3476 & 37118 & 3476 & 193542 & 28550 & 1215038 & 70512 & 166083 & 2844518 \\
\hline 176bit & 7441 & 7150 & 82270 & 7150 & 388870 & 56269 & 5121390 & 145649 & 349258 & 10258362 \\
\hline 192bit & 13691 & 13093 & 154303 & 13093 & 685467 & 96275 & 14109093 & 267084 & 654954 & 29643489 \\
\hline $208 \mathrm{bit}$ & 24430 & 23191 & 299756 & 23191 & 1202798 & 168273 & 24217191 & 471420 & 1177025 & 85199435 \\
\hline beaf $1 \mathrm{w}$ & 500 & 492 & 53403 & 492 & 28073 & 5307 & 452608 & 9814 & 71656 & 114801 \\
\hline c8_mat11 & 5761 & 4562 & 2462970 & 4562 & 78255 & 49640 & 4562 & 95477 & 2621770 & 10254646 \\
\hline connectus & 394707 & 458 & 1127525 & 458 & 478 & 1744 & 1850 & 5185 & 1527818 & 29414 \\
\hline ESOC & 327062 & 37349 & 6019939 & 37349 & 698366 & $>265992$ & 37349 & 776570 & 12993333 & 37844216 \\
\hline EternityII_Etilde & 204304 & 10054 & 1170516 & 10054 & 24368 & 103380 & 4019398 & 205375 & 4202504 & 5074041 \\
\hline $\begin{array}{l}\text { f855_mat9 } \\
\text { - }\end{array}$ & 2511 & 2456 & 171214 & 2456 & 136743 & 26961 & 2456 & 51366 & 264054 & 2893837 \\
\hline GL7d16 & 955127 & 460260 & 14488881 & 460260 & 9346036 & $>320334$ & 4602708 & 9665201 & 36508148 & \\
\hline GL7d17 & 1548649 & 955127 & 25978098 & 955127 & - & $>328160$ & 8596231 & 20057165 & - & - \\
\hline GL7d18 & 1955309 & 1548645 & 35590540 & 1548645 & - & $>352792$ & 17035225 & 32520973 & - & - \\
\hline GL7d19 & 1955296 & 1911130 & 37322725 & 1911130 & - & $>550233$ & 19111408 & 40133312 & - & - \\
\hline GL7d 20 & 1911124 & 1437546 & 29893084 & 1437546 & - & $>527911$ & 8625316 & 30188087 & - & - \\
\hline GL7d 21 & 1437546 & 822922 & 18174775 & 822922 & & $>526636$ & 5760508 & 17281075 & & - \\
\hline GL7d22 & 822906 & 349443 & 8251000 & 349443 & 12616485 & $>535015$ & 2446155 & 7337962 & 30115416 & - \\
\hline GL7d 23 & 349443 & 105054 & 2695430 & 105054 & 1721600 & $>558111$ & 735432 & 2205827 & 10424076 & \\
\hline graphics & 29493 & 11822 & 117954 & 11822 & 31960 & 30470 & 11822 & 24901 & 194127 & 466587 \\
\hline HFE18_96_in & 2372 & 2371 & 933343 & 2371 & 10978 & 26016 & 3376371 & 49576 & 1028976 & 2810425 \\
\hline IG5- 15 & 11369 & 6146 & 323509 & 6146 & 189538 & 62626 & 580318 & 128833 & 643103 & 13575427 \\
\hline IG5-16 & 18846 & 9519 & 588326 & 9519 & 279931 & 97466 & 1470142 & 199678 & 1115361 & 31235491 \\
\hline IG5-17 & 30162 & 14060 & 1035008 & 14060 & 409603 & 145262 & 1758938 & 295028 & 1843157 & 69381526 \\
\hline IG5-18 & 47894 & 20818 & 1790490 & 20818 & 587680 & 216979 & 2260114 & 436954 & 2992054 & 155805819 \\
\hline IMDB & 896302 & 303617 & 3782463 & 303617 & 15909318 & $>487530$ & 303617 & 5858164 & 13438747 & $>4216225900$ \\
\hline kneser_10_4_1 & 349651 & 330751 & 992252 & 330751 & 6394519 & & 330751 & 6759341 & 11405272 & 362087118 \\
\hline landmark & 71952 & 2673 & 1146848 & 2673 & 11307 & 17778 & 75654 & 26909 & 873043 & 378177 \\
\hline LargeRegFile & 2111154 & 801374 & 4944201 & 801374 & 3615761 & $>461966$ & 6411062 & 4106048 & 24923112 & 15941094 \\
\hline Maragal_6 & 21251 & 10144 & 537694 & 10144 & 246737 & 71907 & 5289072 & 212144 & 679769 & 50574842 \\
\hline Maragal_7 & 46845 & 26525 & 1200537 & 26525 & 662167 & 166881 & 5156765 & 553856 & 1641051 & 139030659 \\
\hline Maragal_8 & 60845 & 33093 & 1308415 & 33093 & 1702715 & 229119 & 33093 & 597971 & 1392093 & 88830058 \\
\hline mri1 & 114637 & 65536 & 589824 & 65536 & 519051 & 325965 & 62016508 & 636290 & 1776829 & 8157680 \\
\hline mri2 & 104597 & 63240 & 569160 & 63240 & 1486341 & 392461 & 47669568 & 781491 & 2575072 & 35347277 \\
\hline NotreDame_actors & 383640 & 127823 & 1470404 & 127823 & 6768455 & $>708376$ & 127823 & 2506265 & 6151175 & 1179796747 \\
\hline psseo & 26722 & 11028 & 102432 & 11028 & 31603 & 23833 & 12042028 & 35197 & 180414 & 371305 \\
\hline psse1 & 14318 & 11028 & 57376 & 11028 & 40288 & 28925 & 12042028 & 35771 & 133438 & 381958 \\
\hline $\begin{array}{l}\text { psse2 } \\
\text { psse }\end{array}$ & 28634 & 11028 & 115262 & 11028 & 38437 & 34657 & 11028 & 40814 & 216020 & 394467 \\
\hline rel9 & 5921786 & 274667 & 23667183 & 274667 & 498966 & $>313371$ & 3570851 & 5764774 & & \\
\hline relat9 & 9746232 & 274667 & 38955420 & 274667 & 337475 & $>263449$ & 3845546 & 5763434 & - & - \\
\hline Rucci1 & 1977885 & 109900 & 7791168 & 109900 & 638743 & 932200 & 109900 & 2306811 & 19056222 & 136755107 \\
\hline sls & 1748122 & 62729 & 6804304 & 62729 & 71400 & 108831 & 3013342 & 1226997 & 13636543 & 110829629 \\
\hline TF14 & 3159 & 2644 & 29862 & 2644 & 150764 & 28727 & 3649644 & 55249 & 148306 & 2209059 \\
\hline TF15 & 7741 & 6334 & 80057 & 6334 & 379663 & 69250 & 6334 & 132747 & 368065 & 11277723 \\
\hline TF16 & 19320 & 15437 & 216173 & 15437 & 933646 & 169320 & 15437 & 323886 & 928743 & 64337506 \\
\hline TF17 & 48629 & 38132 & 586218 & 38132 & 2304372 & 418887 & 38132 & 800458 & 2359904 & 360007876 \\
\hline TF18 & 123867 & 95368 & 1597545 & 95368 & 5699962 & $>808291$ & 95368 & 2002386 & 6080905 & $>2147378630$ \\
\hline TF19 & 317955 & 241029 & 4370721 & 241029 & 14260905 & $>811388$ & 241029 & 5061262 & 15718547 & $>8629432746$ \\
\hline tomographic1 & 59360 & 45908 & 647495 & 45908 & 988212 & 280726 & 45908 & 906437 & 2247216 & 31002372 \\
\hline Trec14 & 15904 & 3159 & 2872265 & 3159 & 11942 & 34692 & 2661039 & 66099 & 3251107 & 4909829 \\
\hline wheel_601 & 902103 & 723605 & 2170814 & 723605 & 7796970 & 4253762 & 723605 & 14201674 & 25831230 & \\
\hline
\end{tabular}

ACM Transactions on Mathematical Software, Vol. V, No. N, Article XXXX, Publication date: XXXX 2015. 
Table III: Iterations Required for Subset CUTEst Problems by Each Method.

\begin{tabular}{|c|c|c|c|c|c|c|c|c|c|c|}
\hline name & $m$ & $n$ & $n z(A)$ & no & diagonal & MIQR & RIF & BA-G & MI35 & MI30-MIN \\
\hline BAS1LP & 9825 & 5411 & 587775 & 14838 & 7625 & 48418 & $>741606$ & 4406 & 7977 & 11618 \\
\hline BAXTER & 30733 & 27441 & 111576 & 172315 & $>940092$ & 101 & $>875045$ & $>18992$ & $>322351$ & $>144158$ \\
\hline BCDOUT & 7078 & 5412 & 67344 & 129755 & 122022 & $>452954$ & $>1774726$ & $>73955$ & 142047 & 154786 \\
\hline C09 & 22924 & 10789 & 109651 & 24953 & 5078 & 3612 & 6990 & 2619 & 379 & 194 \\
\hline CONT11_L & 1961394 & 1468599 & 5382999 & 206 & 206 & & 60 & 101 & 22 & 19 \\
\hline DBIR1 & 45775 & 18804 & 1077025 & 1450 & 2228 & 31060 & $>224685$ & 367 & 1858 & $>59884$ \\
\hline DBIR2 & 45877 & 18906 & 1158159 & 19116 & 2211 & 33561 & $>400186$ & 864 & 793 & $>60617$ \\
\hline D2Q06C & 5831 & 2171 & 33081 & 58912 & 1597 & 472 & 12219 & 284 & 209 & 18 \\
\hline DELF000 & 5543 & 3128 & 13741 & 298427 & 26446 & 691 & 30615 & 4665 & 60 & 58 \\
\hline GE & 16369 & 10099 & 44825 & 69740 & 6244 & 573 & 832 & 799 & 28 & 81 \\
\hline LARGE001 & 7176 & 4162 & 18887 & 52556 & 26777 & 53702 & 2233527 & 7422 & 75 & 90 \\
\hline LPL1 & 129959 & 39951 & 386218 & 31755 & 3217 & 564 & $>171967$ & 712 & 420 & 66 \\
\hline MOD2 & 66409 & 34774 & 199810 & 10674 & 1370 & 1515 & 46985 & 579 & 151 & 88 \\
\hline MODEL10 & 16819 & 4400 & 150372 & 34343 & 2229 & 5899 & 131196 & 388 & 744 & 202 \\
\hline MPSBCD03 & 7078 & 5412 & 66210 & 153952 & 149821 & $>450987$ & $>1782418$ & $>70943$ & 145960 & 172717 \\
\hline NSCT2 & 37563 & 23003 & 697738 & 10090 & 1397 & 13476 & $>645872$ & 205 & 610 & $>88277$ \\
\hline NSIR2 & 10057 & 4453 & 154939 & 9622 & 1033 & 20198 & $>1646173$ & 210 & 388 & 76281 \\
\hline PDE1 & 271792 & 270595 & 990587 & 903 & 943 & & & $>2291$ & & \\
\hline PDS-100 & 514577 & 156016 & 1096002 & 682 & 342 & 228 & 203 & 76 & 90 & 64 \\
\hline PDS -90 & 475448 & 142596 & 1014136 & 639 & 331 & 216 & 195 & 73 & 88 & 74 \\
\hline PILOT-JA & 2267 & 940 & 14977 & 137401 & 2346 & 61 & 26428 & 334 & 323 & 54 \\
\hline PILOTNOV & 2446 & 975 & 13331 & 83019 & 1931 & 41 & 18021 & 340 & 214 & 20 \\
\hline RAIL2586 & 923269 & 2586 & 8011362 & 912 & 400 & 809 & 233 & 178 & 151 & 9 \\
\hline RAIL4284 & 1096894 & 4284 & 11284032 & 883 & 732 & 913 & 374 & 212 & 224 & 19 \\
\hline SPAL_004 & 321696 & 10203 & 46168124 & $>5212$ & 3258 & $>5630$ & & 1 & 3764 & $>1397$ \\
\hline STAT96V2 & 957432 & 29089 & 2852184 & 985 & 726 & 464 & 414 & 425 & 19 & 22 \\
\hline STAT96V3 & 1113780 & 33841 & 3317736 & 1054 & 765 & 484 & 433 & 414 & 20 & 27 \\
\hline STAT96V4 & 63076 & 3173 & 491336 & 4087 & 809 & 1757 & 449 & 125 & 17 & 24 \\
\hline STORMG21K & 1377306 & 526185 & 3459881 & 1401 & 183 & $>7602$ & & $>919$ & 2281 & $>3327$ \\
\hline WATSON_1 & 386992 & 201155 & 1055093 & 2161 & 422 & 165 & 249 & 56 & 73 & 8 \\
\hline WATSON_2 & 677224 & 352013 & 1846391 & 1812 & 349 & 119 & 185 & 48 & 54 & 7 \\
\hline WORLD & 67147 & 34506 & 198883 & 9814 & 1369 & 1084 & 24839 & 571 & 154 & 70 \\
\hline
\end{tabular}


Table III: Iterations Required for Subset UF Problems by Each Method.

\begin{tabular}{|c|c|c|c|c|c|c|c|c|c|c|}
\hline name & $m$ & $n$ & $n z(A)$ & no & diagonal & MIQR & RIF & BA-G & MI35 & MI30-MIN \\
\hline 12month1 & 872622 & 12471 & 22624727 & $>9415$ & 266 & 973 & & 60 & 369 & 293 \\
\hline $162 \mathrm{bit}$ & 3606 & 3476 & 37118 & 29440 & 2540 & 729 & 1493 & 319 & 247 & 252 \\
\hline 176bit & 7441 & 7150 & 82270 & 147449 & 6538 & 2697 & 3892 & 655 & 454 & 981 \\
\hline 192bit & 13691 & 13093 & 154303 & 326126 & 12200 & 4800 & 7238 & 6005 & 1281 & 1519 \\
\hline $208 \mathrm{bit}$ & 24430 & 23191 & 299756 & 606439 & 17074 & 9741 & 13993 & 6219 & 2197 & 3132 \\
\hline beaf $1 \mathrm{w}$ & 500 & 492 & 53403 & 43174 & 41103 & 38035 & $>5$ & 469 & 33910 & $>2951677$ \\
\hline c8_mat11 & 5761 & 4562 & 2462970 & 40930 & 38679 & $>176239$ & $>193279$ & $>7647$ & 30090 & $>67344$ \\
\hline connectus & 394707 & 458 & 1127525 & 1753 & 7 & 114 & 7 & 3 & 6 & 3 \\
\hline ESOC & 327062 & 37349 & 6019939 & 5596 & 15011 & $>20554$ & - & $>2671$ & $>21840$ & $>9045$ \\
\hline EternityII_Etilde & 204304 & 10054 & 1170516 & 1356 & 1122 & 2098 & 883 & 384 & 585 & 81 \\
\hline f855_mat9 & 2511 & 2456 & 171214 & 19082 & 20175 & 267667 & 321572 & $>72874$ & 12324 & $>832602$ \\
\hline GL7d16 & 955127 & 460260 & 14488881 & 61 & 48 & 265 & - & 9 & 32 & 35 \\
\hline GL7d17 & 1548649 & 955127 & 25978098 & 58 & 48 & - & - & 8 & 28 & - \\
\hline GL7d18 & 1955309 & 1548645 & 35590540 & 79 & 64 & - & - & 10 & 40 & - \\
\hline GL7d19 & 1955296 & 1911130 & 37322725 & 204 & 53 & - & - & 9 & 46 & - \\
\hline GL7d 20 & 1911124 & 1437546 & 29893084 & 137 & 31 & - & - & 5 & 28 & - \\
\hline GL7d21 & 1437546 & 822922 & 18174775 & 143 & 26 & & - & 6 & 25 & \\
\hline GL7d22 & 822906 & 349443 & 8251000 & 238 & 24 & 124 & - & 6 & 22 & 30 \\
\hline GL7d 23 & 349443 & 105054 & 2695430 & 340 & 24 & 91 & - & 6 & 21 & 22 \\
\hline graphics & 29493 & 11822 & 117954 & $>1623343$ & 302842 & 312745 & 177862 & $>44385$ & 1908 & 221 \\
\hline HFE18_96_in & 2372 & 2371 & 933343 & 30425 & 15101 & 30092 & 12419 & 1632 & 14635 & 16052 \\
\hline IG5-15 & 11369 & 6146 & 323509 & 4567 & 610 & 126 & 323 & 92 & 240 & 533 \\
\hline IG5-16 & 18846 & 9519 & 588326 & 7421 & 866 & 150 & 478 & 151 & 347 & 771 \\
\hline IG5-17 & 30162 & 14060 & 1035008 & 7243 & 828 & 169 & 411 & 123 & 329 & 705 \\
\hline IG5-18 & 47894 & 20818 & 1790490 & 7281 & 735 & 205 & 446 & 107 & 312 & 889 \\
\hline IMDB & 896302 & 303617 & 3782463 & $>15970$ & $>15288$ & $>3043$ & - & $>899$ & $>8031$ & $>3368$ \\
\hline kneser_10_4_1 & 349651 & 330751 & 992252 & 17207 & 10782 & $>9032$ & - & $>1755$ & 3258 & 1866 \\
\hline landmark & 71952 & 2673 & 1146848 & 19557 & 894 & 36 & 274 & 27 & 12 & 25 \\
\hline LargeRegFile & 2111154 & 801374 & 4944201 & 784 & 54 & 168 & & 7 & 12 & 21 \\
\hline Maragal_6 & 21251 & 10144 & 537694 & 5400 & 3254 & 10031 & $>579407$ & 496 & 1016 & 1756 \\
\hline Maragal_7 & 46845 & 26525 & 1200537 & 7469 & 2683 & 5150 & $>1913$ & 192 & 680 & 1112 \\
\hline Maragal_8 & 60845 & 33093 & 1308415 & $>134737$ & $>131006$ & $>43588$ & $>103951$ & $>8548$ & $>91721$ & $>52936$ \\
\hline mri1 & 114637 & 65536 & 589824 & 6150 & 6103 & 8744 & 1603 & 932 & 2217 & 65 \\
\hline mri2 & 104597 & 63240 & 569160 & 11848 & 11853 & 4126 & $>1$ & 744 & 2933 & 10317 \\
\hline NotreDame_actors & 383640 & 127823 & 1470404 & $>64753$ & $>63129$ & $>8938$ & & $>3314$ & $>26248$ & $>9268$ \\
\hline psse0 & 26722 & 11028 & 102432 & 190067 & 41438 & 1564 & 20222 & 40001 & 104 & 26 \\
\hline psse1 & 14318 & 11028 & 57376 & 190737 & 58420 & 5113 & $>1072010$ & 51952 & 665 & 138 \\
\hline $\begin{array}{l}\text { psse2 } \\
\text { psse }\end{array}$ & 28634 & 11028 & 115262 & 191582 & 51664 & 7598 & $>887601$ & $>51962$ & 687 & 161 \\
\hline rel9 & 5921786 & 274667 & 23667183 & 110 & 81 & 107 & - & 12 & 37 & \\
\hline relat9 & 9746232 & 274667 & 38955420 & 88 & 76 & 82 & - & 13 & 36 & - \\
\hline Rucci1 & 1977885 & 109900 & 7791168 & $>13607$ & 8330 & $>13414$ & 1822 & $>1813$ & $>12647$ & 555 \\
\hline sls & 1748122 & 62729 & 6804304 & 610 & 188 & 619 & & 47 & 68 & 13 \\
\hline TF14 & 3159 & 2644 & 29862 & 34774 & 25705 & 44459 & 12884 & 1758 & 11389 & 1091 \\
\hline TF15 & 7741 & 6334 & 80057 & 107320 & 81868 & 246138 & 43583 & $>63793$ & 41012 & 1723 \\
\hline TF16 & 19320 & 15437 & 216173 & 323986 & 227796 & $>107703$ & 142169 & $>29019$ & 144257 & 1102 \\
\hline TF17 & 48629 & 38132 & 586218 & $>440453$ & $>465868$ & $>43370$ & $>182890$ & $>10465$ & $>145969$ & 1212 \\
\hline TF18 & 123867 & 95368 & 1597545 & $>146295$ & $>130859$ & $>17063$ & - & $>3960$ & $>50392$ & 1469 \\
\hline TF19 & 317955 & 241029 & 4370721 & $>42857$ & $>44132$ & $>5160$ & & $>1478$ & $>14317$ & 1086 \\
\hline tomographic1 & 59360 & 45908 & 647495 & 65478 & 18912 & $>75149$ & $>157297$ & $>8915$ & 1867 & 1837 \\
\hline Trec14 & 15904 & 3159 & 2872265 & 2005 & 1600 & 8542 & $>1$ & 690 & 1598 & 16105 \\
\hline wheel_601 & 902103 & 723605 & 2170814 & $>21448$ & $>21212$ & $>4579$ & $>7467$ & $>762$ & $>6083$ & $>3554$ \\
\hline
\end{tabular}

ACM Transactions on Mathematical Software, Vol. V, No. N, Article XXXX, Publication date: XXXX 2015. 
Table IV: Time Required for Factors for Subset CUTEst Problems by Each Method.

\begin{tabular}{|c|c|c|c|c|c|c|c|c|c|}
\hline name & $m$ & $n$ & $n z(A)$ & diagonal & MIQR & RIF & MI35 & MI30-MIN & MA87 \\
\hline BAS1LP & 9825 & 5411 & 587775 & 0.00 & 0.66 & 7.30 & 1.35 & 2.07 & 0.95 \\
\hline BAXTER & 30733 & 27441 & 111576 & 0.00 & 0.31 & 1.34 & 0.28 & 0.98 & 0.38 \\
\hline BCDOUT & 7078 & 5412 & 67344 & 0.00 & 0.40 & 1.51 & 0.46 & 0.29 & 0.39 \\
\hline $\mathrm{COP}$ & 22924 & 10789 & 109651 & 0.00 & 0.17 & 1.06 & 0.21 & 0.99 & 0.10 \\
\hline CONT11_L & 1961394 & 1468599 & 5382999 & 0.02 & $>15.95$ & 45.92 & 9.15 & 20.14 & 8.38 \\
\hline DBIR1 & 45775 & 18804 & 1077025 & 0.00 & 0.82 & 42.48 & 2.14 & 5.12 & 0.99 \\
\hline DBIR2 & 45877 & 18906 & 1158159 & 0.00 & 0.84 & 49.64 & 1.90 & 4.38 & 1.11 \\
\hline D2Q06C & 5831 & 2171 & 33081 & 0.00 & 0.03 & 0.06 & 0.03 & 0.17 & 0.03 \\
\hline DELFO00 & 5543 & 3128 & 13741 & 0.00 & 0.01 & 0.01 & 0.04 & 0.01 & 0.02 \\
\hline GE & 16369 & 10099 & 44825 & 0.00 & 0.06 & 0.17 & 0.08 & 0.39 & 0.06 \\
\hline LARGE001 & 7176 & 4162 & 18887 & 0.00 & 0.01 & 0.01 & 0.01 & 0.02 & 0.02 \\
\hline LPL1 & 129959 & 39951 & 386218 & 0.00 & 0.26 & 0.44 & 0.92 & 3.59 & 0.38 \\
\hline MOD2 & 66409 & 34774 & 199810 & 0.00 & 0.43 & 2.23 & 0.98 & 1.31 & 0.29 \\
\hline MODEL10 & 16819 & 4400 & 150372 & 0.00 & 0.20 & 0.40 & 0.09 & 0.53 & 0.08 \\
\hline MPSBCD03 & 7078 & 5412 & 66210 & 0.00 & 0.39 & 1.57 & 0.42 & 0.37 & 0.40 \\
\hline NSCT2 & 37563 & 23003 & 697738 & 0.00 & 1.42 & 38.78 & 2.51 & 4.21 & 2.05 \\
\hline NSIR2 & 10057 & 4453 & 154939 & 0.00 & 0.21 & 2.49 & 0.21 & 0.18 & 0.15 \\
\hline PDE1 & 271792 & 270595 & 990587 & 0.00 & $>591.48$ & $>600.02$ & $>0.01$ & $>600.82$ & $>0.01$ \\
\hline PDS- 100 & 514577 & 156016 & 1096002 & 0.00 & 1.17 & 22.99 & 1.55 & 13.34 & 2.53 \\
\hline PDS-90 & 475448 & 142596 & 1014136 & 0.00 & 1.07 & 21.69 & 1.43 & 12.40 & 2.40 \\
\hline PILOT-JA & 2267 & 940 & 14977 & 0.00 & 0.02 & 0.04 & 0.02 & 0.02 & 0.02 \\
\hline PILOTNOV & 2446 & 975 & 13331 & 0.00 & 0.02 & 0.03 & 0.02 & 0.02 & 0.02 \\
\hline RAIL2586 & 923269 & 2586 & 8011362 & 0.01 & 2.33 & 110.81 & 2.17 & 3.83 & 1.25 \\
\hline RAIL4284 & 1096894 & 4284 & 11284032 & 0.02 & 4.75 & 352.77 & 4.18 & 11.91 & 2.46 \\
\hline SPAL_004 & 321696 & 10203 & 46168124 & 0.08 & 9.61 & $>601.39$ & 71.76 & 43.20 & 73.46 \\
\hline STAT96V2 & 957432 & 29089 & 2852184 & 0.01 & 0.15 & 0.65 & 0.27 & 1.12 & 0.26 \\
\hline STAT96V3 & 1113780 & 33841 & 3317736 & 0.01 & 0.17 & 0.78 & 0.32 & 1.32 & 0.37 \\
\hline STAT96V4 & 63076 & 3173 & 491336 & 0.00 & 0.04 & 2.36 & 0.08 & 0.22 & 0.05 \\
\hline STORMG21K & 1377306 & 526185 & 3459881 & 0.01 & 42.59 & $>600.04$ & 30.21 & 62.51 & 113.37 \\
\hline WATSON_1 & 386992 & 201155 & 1055093 & 0.00 & 1.69 & 0.53 & 1.48 & 1.02 & 1.15 \\
\hline WATSON_2 & 677224 & 352013 & 1846391 & 0.01 & 5.04 & 4.22 & 2.85 & 1.97 & 2.21 \\
\hline WORLD & 67147 & 34506 & 198883 & 0.00 & 0.41 & 2.17 & 0.74 & 3.73 & 0.29 \\
\hline
\end{tabular}


Table IV: Time Required for Factors for Subset UF Problems by Each Method.

\begin{tabular}{|c|c|c|c|c|c|c|c|c|c|}
\hline name & $m$ & $n$ & $n z(A)$ & diagonal & MIQR & RIF & MI35 & MI30-MIN & MA87 \\
\hline 12month1 & 872622 & 12471 & 22624727 & 0.04 & 15.03 & $>600.67$ & 170.63 & 363.98 & 117.64 \\
\hline $162 \mathrm{bit}$ & 3606 & 3476 & 37118 & 0.00 & 0.43 & 1.80 & 0.13 & 0.17 & 0.14 \\
\hline $176 \mathrm{bit}$ & 7441 & 7150 & 82270 & 0.00 & 1.16 & 8.51 & 0.28 & 0.47 & 0.64 \\
\hline $192 \mathrm{bit}$ & 13691 & 13093 & 154303 & 0.00 & 2.40 & 30.75 & 0.46 & 0.75 & 2.54 \\
\hline 208bit & 24430 & 23191 & 299756 & 0.00 & 4.77 & 109.27 & 0.98 & 1.91 & 11.70 \\
\hline beaflw & 500 & 492 & 53403 & 0.00 & 0.06 & 0.18 & 0.09 & 0.12 & 0.07 \\
\hline c8_mat11 & 5761 & 4562 & 2462970 & 0.01 & 4.37 & 66.65 & 30.65 & 8.03 & 23.21 \\
\hline connectus & 394707 & 458 & 1127525 & 0.00 & 0.11 & 2.32 & 0.22 & 0.60 & 0.17 \\
\hline ESOC & 327062 & 37349 & 6019939 & 0.01 & 6.34 & $>600.14$ & 3.35 & 340.01 & 3.94 \\
\hline EternityII_Etilde & 204304 & 10054 & 1170516 & 0.00 & 0.63 & 38.64 & 1.14 & 2.25 & 0.47 \\
\hline f855_mat9 & 2511 & 2456 & 171214 & 0.00 & 0.63 & 3.01 & 0.88 & 0.55 & 0.74 \\
\hline GL7d16 & 955127 & 460260 & 14488881 & 0.03 & 278.05 & $>600.74$ & 74.74 & 229.19 & $>600.86$ \\
\hline GL7d17 & 1548649 & 955127 & 25978098 & 0.06 & $>600.05$ & $>601.73$ & 311.42 & $>600.18$ & $>600.11$ \\
\hline GL7d18 & 1955309 & 1548645 & 35590540 & 0.08 & $>600.00$ & $>602.63$ & $>600.40$ & $>600.43$ & $>600.59$ \\
\hline GL7d19 & 1955296 & 1911130 & 37322725 & 0.08 & $>600.32$ & $>602.93$ & $>600.46$ & $>600.00$ & $>600.45$ \\
\hline GL7d20 & 1911124 & 1437546 & 29893084 & 0.07 & $>600.27$ & $>602.04$ & 412.34 & $>600.59$ & $>600.22$ \\
\hline GL7d21 & 1437546 & 822922 & 18174775 & 0.04 & $>600.30$ & $>601.12$ & 75.82 & $>600.79$ & $>600.90$ \\
\hline GL7d22 & 822906 & 349443 & 8251000 & 0.02 & 167.10 & $>600.39$ & 22.34 & 198.70 & $>600.65$ \\
\hline GL7d23 & 349443 & 105054 & 2695430 & 0.01 & 23.48 & $>600.09$ & 5.26 & 98.90 & $>600.43$ \\
\hline graphics & 29493 & 11822 & 117954 & 0.00 & 0.09 & 0.17 & 0.04 & 0.08 & 0.06 \\
\hline HFE18_96_in & 2372 & 2371 & 933343 & 0.00 & 1.13 & 14.67 & 5.80 & 0.76 & 3.31 \\
\hline IG5-15 & 11369 & 6146 & 323509 & 0.00 & 0.99 & 9.91 & 0.70 & 1.98 & 1.50 \\
\hline IG5-16 & 18846 & 9519 & 588326 & 0.00 & 1.84 & 27.11 & 1.68 & 1.59 & 3.75 \\
\hline IG5-17 & 30162 & 14060 & 1035008 & 0.00 & 3.26 & 70.73 & 2.72 & 7.52 & 11.09 \\
\hline IG5-18 & 47894 & 20818 & 1790490 & 0.00 & 5.88 & 182.75 & 6.82 & 10.52 & 38.25 \\
\hline IMDB & 896302 & 303617 & 3782463 & 0.01 & 51.43 & $>600.23$ & 40.43 & 131.77 & $>205.71$ \\
\hline kneser_10_4_1 & 349651 & 330751 & 992252 & 0.00 & 9.22 & $>20.61$ & 5.69 & 15.88 & 31.95 \\
\hline landmark & 71952 & 2673 & 1146848 & 0.00 & 0.27 & 1.12 & 0.28 & 0.52 & 0.18 \\
\hline LargeRegFile & 2111154 & 801374 & 4944201 & 0.01 & 10.46 & $>600.08$ & 2.50 & 57.99 & 127.40 \\
\hline Maragal_6 & 21251 & 10144 & 537694 & 0.00 & 2.41 & 41.79 & 13.15 & 2.31 & 17.11 \\
\hline Maragal_7 & 46845 & 26525 & 1200537 & 0.00 & 8.64 & 145.90 & 45.06 & 8.91 & 56.28 \\
\hline Maragal_8 & 60845 & 33093 & 1308415 & 0.00 & 16.17 & 205.65 & 5.18 & 3.10 & 14.09 \\
\hline mri1 & 114637 & 65536 & 589824 & 0.00 & 1.25 & 95.20 & 2.23 & 2.07 & 0.53 \\
\hline mri2 & 104597 & 63240 & 569160 & 0.00 & 4.07 & 68.05 & 4.84 & 6.73 & 3.98 \\
\hline NotreDame_actors & 383640 & 127823 & 1470404 & 0.01 & 11.45 & $>600.04$ & 9.09 & 31.35 & 591.52 \\
\hline psse0 & 26722 & 11028 & 102432 & 0.00 & 0.07 & 0.04 & 0.02 & 0.07 & 0.14 \\
\hline psse1 & 14318 & 11028 & 57376 & 0.00 & 0.09 & 0.05 & 0.03 & 0.03 & 0.03 \\
\hline psse2 & 28634 & 11028 & 115262 & 0.00 & 0.08 & 0.13 & 0.04 & 0.06 & 0.04 \\
\hline rel9 & 5921786 & 274667 & 23667183 & 0.04 & 15.81 & $>601.16$ & 56.51 & $>600.26$ & $>600.42$ \\
\hline relat9 & 9746232 & 274667 & 38955420 & 0.07 & 14.14 & $>602.27$ & 62.22 & $>600.49$ & $>600.26$ \\
\hline Rucci1 & 1977885 & 109900 & 7791168 & 0.01 & 2.40 & 179.08 & 2.55 & 39.35 & 7.97 \\
\hline sls & 1748122 & 62729 & 6804304 & 0.01 & 1.60 & $>601.94$ & 6.62 & 14.26 & 24.23 \\
\hline TF14 & 3159 & 2644 & 29862 & 0.00 & 0.23 & 0.43 & 0.06 & 0.21 & 0.11 \\
\hline TF15 & 7741 & 6334 & 80057 & 0.00 & 0.76 & 2.66 & 0.20 & 0.46 & 0.59 \\
\hline TF16 & 19320 & 15437 & 216173 & 0.00 & 2.47 & 17.64 & 0.45 & 1.17 & 5.74 \\
\hline TF17 & 48629 & 38132 & 586218 & 0.00 & 7.66 & 120.76 & 1.21 & 4.23 & 77.31 \\
\hline TF18 & 123867 & 95368 & 1597545 & 0.00 & 24.45 & $>600.03$ & 3.50 & 13.48 & $>21.69$ \\
\hline TF19 & 317955 & 241029 & 4370721 & 0.01 & 84.67 & $>600.09$ & 10.60 & 40.42 & $>224.19$ \\
\hline tomographic1 & 59360 & 45908 & 647495 & 0.00 & 2.92 & 3.12 & 1.46 & 1.61 & 1.52 \\
\hline Trec14 & 15904 & 3159 & 2872265 & 0.00 & 2.39 & 58.89 & 26.88 & 11.27 & 19.05 \\
\hline wheel_601 & 902103 & 723605 & 2170814 & 0.01 & 20.63 & 39.95 & 30.90 & 49.67 & $>600.25$ \\
\hline
\end{tabular}


Table V: Total Time Required for Subset CUTEst Problems by Each Method.

\begin{tabular}{|c|c|c|c|c|c|c|c|c|c|c|c|}
\hline name & $m$ & $n$ & $n z(A)$ & no & diagonal & MIQR & RIF & BA-G & MI35 & MI30-MIN & MA87 \\
\hline BAS1LP & 9825 & 5411 & 587775 & 8.37 & 5.80 & 74.22 & $>600.33$ & 92.27 & 8.77 & 49.65 & 0.99 \\
\hline BAXTER & 30733 & 27441 & 111576 & 73.77 & $>600.00$ & 0.51 & $>601.34$ & $>600.01$ & $>600.28$ & $>600.33$ & 0.40 \\
\hline BCDOUT & 7078 & 5412 & 67344 & 15.18 & 18.57 & $>600.40$ & $>601.51$ & $>600.00$ & 64.14 & 142.05 & 0.40 \\
\hline C09 & 22924 & 10789 & 109651 & 6.59 & 1.69 & 4.68 & 5.62 & 32.24 & 0.53 & 1.53 & 0.10 \\
\hline CONT11_L & 1961394 & 1468599 & 5382999 & 9.58 & 11.24 & & 51.37 & 39.39 & 11.83 & 25.45 & 9.65 \\
\hline DBIR1 & 45775 & 18804 & 1077025 & 2.91 & 4.87 & 131.85 & $>600.49$ & 8.02 & 8.02 & $>600.27$ & 1.03 \\
\hline DBIR2 & 45877 & 18906 & 1158159 & 43.93 & 5.16 & 148.55 & $>600.67$ & 26.50 & 4.40 & $>600.46$ & 1.15 \\
\hline D2Q06C & 5831 & 2171 & 33081 & 3.70 & 0.12 & 0.16 & 2.03 & 0.48 & 0.07 & 0.20 & 0.03 \\
\hline DELF000 & 5543 & 3128 & 13741 & 16.12 & 1.98 & 0.14 & 4.38 & 27.21 & 0.05 & 0.03 & 0.03 \\
\hline GE & 16369 & 10099 & 44825 & 11.81 & 1.46 & 0.60 & 0.61 & 7.31 & 0.11 & 0.54 & 0.06 \\
\hline LARGE001 & 7176 & 4162 & 18887 & 3.65 & 2.51 & 13.35 & 427.11 & 38.54 & 0.03 & 0.06 & 0.03 \\
\hline LPL1 & 129959 & 39951 & 386218 & 62.09 & 6.99 & 2.76 & $>600.45$ & 30.19 & 2.85 & 4.46 & 0.41 \\
\hline MOD2 & 66409 & 34774 & 199810 & 7.86 & 1.28 & 7.44 & 99.95 & 14.93 & 1.44 & 2.06 & 0.31 \\
\hline MODEL10 & 16819 & 4400 & 150372 & 6.23 & 0.48 & 3.06 & 47.21 & 1.55 & 0.44 & 0.87 & 0.08 \\
\hline MPSBCD03 & 7078 & 5412 & 66210 & 17.87 & 22.69 & $>600.39$ & $>601.57$ & $>600.00$ & 65.92 & 158.56 & 0.41 \\
\hline NSCT2 & 37563 & 23003 & 697738 & 12.03 & 1.88 & 67.30 & $>600.79$ & 2.90 & 3.84 & $>600.28$ & 2.10 \\
\hline NSIR2 & 10057 & 4453 & 154939 & 2.19 & 0.17 & 16.27 & $>600.50$ & 0.66 & 0.38 & 104.00 & 0.15 \\
\hline PDE1 & 271792 & 270595 & 990587 & 5.79 & 7.59 & & & $>600.05$ & & & \\
\hline PDS-100 & 514577 & 156016 & 1096002 & 7.75 & 3.88 & 5.51 & 25.92 & 3.82 & 3.53 & 17.60 & 2.73 \\
\hline PDS -90 & 475448 & 142596 & 1014136 & 6.45 & 3.56 & 4.80 & 24.24 & 3.47 & 3.12 & 16.83 & 2.57 \\
\hline PILOT-JA & 2267 & 940 & 14977 & 4.00 & 0.08 & 0.04 & 1.71 & 0.17 & 0.08 & 0.04 & 0.02 \\
\hline PILOTNOV & 2446 & 975 & 13331 & 2.35 & 0.07 & 0.03 & 1.17 & 0.14 & 0.04 & 0.03 & 0.02 \\
\hline RAIL2586 & 923269 & 2586 & 8011362 & 31.83 & 14.22 & 25.87 & 117.90 & 47.76 & 6.58 & 4.79 & 1.54 \\
\hline RAIL4284 & 1096894 & 4284 & 11284032 & 41.94 & 35.47 & 44.83 & 369.62 & 88.69 & 14.09 & 14.59 & 2.91 \\
\hline SPAL_004 & 321696 & 10203 & 46168124 & $>600.18$ & 374.89 & $>600.81$ & & 4.76 & 466.94 & $>600.72$ & 74.99 \\
\hline STAT96V2 & 957432 & 29089 & 2852184 & 12.98 & 9.55 & 6.27 & 6.09 & 16.31 & 0.55 & 2.39 & 0.35 \\
\hline STAT96V3 & 1113780 & 33841 & 3317736 & 16.34 & 11.98 & 7.63 & 7.46 & 18.75 & 0.65 & 3.13 & 0.48 \\
\hline STAT96V4 & 63076 & 3173 & 491336 & 3.48 & 0.69 & 1.64 & 2.75 & 1.53 & 0.10 & 0.32 & 0.06 \\
\hline STORMG21K & 1377306 & 526185 & 3459881 & 33.31 & 5.09 & $>600.67$ & & $>600.94$ & 167.08 & $>600.67$ & 115.19 \\
\hline WATSON_1 & 386992 & 201155 & 1055093 & 14.62 & 3.25 & 4.28 & 3.27 & 3.77 & 2.83 & 1.40 & 1.25 \\
\hline WATSON_2 & 677224 & 352013 & 1846391 & 22.86 & 5.03 & 9.25 & 8.22 & 5.98 & 4.67 & 2.59 & 2.38 \\
\hline WORLD & 67147 & 34506 & 198883 & 6.57 & 1.24 & 5.23 & 54.05 & 14.52 & 1.24 & 4.33 & 0.31 \\
\hline
\end{tabular}


Table V: Total Time Required for Subset UF Problems by Each Method.

\begin{tabular}{|c|c|c|c|c|c|c|c|c|c|c|c|}
\hline name & $m$ & $n$ & $n z(A)$ & no & diagonal & MIQR & RIF & BA-G & MI35 & MI30-MIN & MA87 \\
\hline 12month1 & 872622 & 12471 & 22624727 & $>600.12$ & 17.11 & 73.17 & & 29.84 & 192.66 & 419.45 & 118.47 \\
\hline $162 \mathrm{bit}$ & 3606 & 3476 & 37118 & 2.77 & 0.33 & 1.24 & 2.18 & 0.67 & 0.18 & 0.34 & 0.15 \\
\hline $176 \mathrm{bit}$ & 7441 & 7150 & 82270 & 32.68 & 1.77 & 7.35 & 10.64 & 4.98 & 0.60 & 1.88 & 0.66 \\
\hline $192 \mathrm{bit}$ & 13691 & 13093 & 154303 & 145.33 & 6.80 & 23.16 & 38.23 & 107.45 & 2.19 & 5.50 & 2.61 \\
\hline $208 \mathrm{bit}$ & 24430 & 23191 & 299756 & 566.32 & 20.49 & 83.52 & 137.37 & 215.02 & 7.12 & 23.16 & 11.88 \\
\hline beaf1w & 500 & 492 & 53403 & 1.83 & 1.85 & 7.26 & $>0.18$ & 0.50 & 2.61 & $>600.61$ & 0.08 \\
\hline c8_mat 11 & 5761 & 4562 & 2462970 & 123.49 & 116.55 & $>600.38$ & $>600.66$ & $>600.03$ & 125.79 & $>600.84$ & 23.29 \\
\hline connectus & 394707 & 458 & 1127525 & 10.86 & 0.05 & 0.75 & 2.37 & 0.16 & 0.26 & 0.71 & 0.20 \\
\hline ESOC & 327062 & 37349 & 6019939 & 152.70 & 398.52 & $>600.39$ & 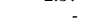 & $>600.23$ & $>603.38$ & $>600.05$ & 4.21 \\
\hline EternityII_Etilde & 204304 & 10054 & 1170516 & 6.88 & 5.81 & 10.79 & 43.13 & 7.94 & 4.16 & 4.36 & 0.52 \\
\hline f855_mat9 & 2511 & 2456 & 171214 & 2.08 & 2.54 & 219.75 & 77.67 & $>600.01$ & 4.25 & $>600.44$ & 0.75 \\
\hline GL7d16 & 955127 & 460260 & 14488881 & 7.11 & 5.82 & 333.57 & & 7.04 & 80.55 & 246.11 & \\
\hline GL7d17 & 1548649 & 955127 & 25978098 & 14.63 & 12.47 & 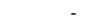 & - & 15.01 & 323.25 & 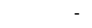 & - \\
\hline GL7d18 & 1955309 & 1548645 & 35590540 & 30.68 & 25.32 & - & - & 26.39 & - & - & - \\
\hline GL7d19 & 1955296 & 1911130 & 37322725 & 79.19 & 21.12 & - & - & 38.93 & - & - & - \\
\hline GL7d20 & 1911124 & 1437546 & 29893084 & 43.87 & 10.14 & - & - & 23.70 & 428.69 & - & - \\
\hline GL7d 21 & 1437546 & 822922 & 18174775 & 24.30 & 4.82 & & - & 10.40 & 83.70 & & - \\
\hline GL7d22 & 822906 & 349443 & 8251000 & 14.51 & 1.63 & 191.82 & - & 3.51 & 25.00 & 210.17 & - \\
\hline GL7d 23 & 349443 & 105054 & 2695430 & 4.70 & 0.43 & 27.15 & - & 0.97 & 5.88 & 101.50 & - \\
\hline graphics & 29493 & 11822 & 117954 & $>600.00$ & 131.90 & 266.73 & 117.27 & $>600.00$ & 1.42 & 0.51 & 0.07 \\
\hline HFE18_96_in & 2372 & 2371 & 933343 & 28.72 & 12.30 & 31.62 & 25.68 & 51.44 & 19.44 & 54.85 & 3.34 \\
\hline IG5-15 & 11369 & 6146 & 323509 & 2.51 & 0.31 & 1.19 & 10.19 & 0.88 & 0.91 & 3.44 & 1.54 \\
\hline IG5-16 & 18846 & 9519 & 588326 & 8.14 & 0.96 & 2.27 & 27.85 & 2.64 & 2.27 & 5.54 & 3.82 \\
\hline IG5-17 & 30162 & 14060 & 1035008 & 19.59 & 1.92 & 4.11 & 71.91 & 3.90 & 3.81 & 14.00 & 11.25 \\
\hline IG5-18 & 47894 & 20818 & 1790490 & 47.51 & 4.77 & 7.96 & 185.81 & 8.09 & 9.31 & 28.20 & 38.58 \\
\hline IMDB & 896302 & 303617 & 3782463 & $>600.08$ & $>600.06$ & $>600.69$ & - & $>600.35$ & $>640.51$ & $>600.87$ & \\
\hline kneser_10_4__1 & 349651 & 330751 & 992252 & 162.24 & 120.85 & $>600.28$ & - & $>600.14$ & 138.66 & 152.51 & 32.83 \\
\hline landmark & 71952 & 2673 & 1146848 & 33.28 & 1.67 & 0.34 & 1.58 & 1.85 & 0.31 & 0.69 & 0.21 \\
\hline LargeRegFile & 2111154 & 801374 & 4944201 & 42.50 & 3.00 & 24.82 & & 3.08 & 3.66 & 67.27 & 127.83 \\
\hline Maragal_6 & 21251 & 10144 & 537694 & 3.78 & 2.11 & 24.19 & $>600.80$ & 4.42 & 14.48 & 7.85 & 17.22 \\
\hline Maragal_-7 & 46845 & 26525 & 1200537 & 16.32 & 6.08 & 41.25 & $>151.90$ & 5.28 & 47.83 & 18.50 & 56.57 \\
\hline Maragal_8 & 60845 & 33093 & 1308415 & $>600.01$ & $>600.01$ & $>600.18$ & $>600.66$ & $>600.07$ & $>605.19$ & $>600.18$ & 14.30 \\
\hline mri1 & 114637 & 65536 & 589824 & 13.39 & 15.33 & 60.08 & 102.66 & 68.69 & 16.27 & 2.99 & 0.58 \\
\hline mri2 & 104597 & 63240 & 569160 & 20.90 & 24.62 & 49.53 & $>68.07$ & 43.48 & 22.23 & 156.20 & 4.07 \\
\hline NotreDame_actors & 383640 & 127823 & 1470404 & $>600.02$ & $>600.02$ & $>600.52$ & & $>600.05$ & $>609.12$ & $>600.39$ & 593.90 \\
\hline psse0 & 26722 & 11028 & 102432 & 49.79 & 13.48 & 1.28 & 11.99 & 358.86 & 0.09 & 0.12 & 0.14 \\
\hline psse1 & 14318 & 11028 & 57376 & 38.77 & 15.38 & 3.96 & $>600.05$ & 509.79 & 0.41 & 0.19 & 0.04 \\
\hline psse2 & 28634 & 11028 & 115262 & 56.29 & 17.98 & 6.44 & $>600.13$ & $>600.01$ & 0.49 & 0.37 & 0.05 \\
\hline rel9 & 5921786 & 274667 & 23667183 & 25.00 & 16.49 & 38.17 & & 24.04 & 66.04 & 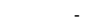 & - \\
\hline relat9 & 9746232 & 274667 & 38955420 & 33.85 & 26.92 & 42.44 & & 39.81 & 76.63 & & 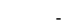 \\
\hline Rucci1 & 1977885 & 109900 & 7791168 & $>600.05$ & 343.67 & $>600.47$ & 261.20 & $>600.42$ & $>602.63$ & 133.04 & 8.51 \\
\hline sls & 1748122 & 62729 & 6804304 & 30.31 & 8.59 & 30.25 & & 9.41 & 10.11 & 16.99 & 24.94 \\
\hline TF14 & 3159 & 2644 & 29862 & 1.84 & 1.83 & 36.66 & 2.71 & 7.02 & 2.53 & 0.76 & 0.11 \\
\hline TF15 & 7741 & 6334 & 80057 & 14.57 & 14.46 & 513.65 & 21.79 & $>600.01$ & 21.91 & 2.64 & 0.62 \\
\hline TF16 & 19320 & 15437 & 216173 & 110.15 & 99.55 & $>600.48$ & 172.42 & $>600.00$ & 195.85 & 5.27 & 5.87 \\
\hline TF17 & 48629 & 38132 & 586218 & $>600.00$ & $>600.01$ & $>600.68$ & $>600.77$ & $>600.01$ & $>601.21$ & 17.34 & 77.98 \\
\hline TF18 & 123867 & 95368 & 1597545 & $>600.01$ & $>600.01$ & $>600.49$ & - & $>600.01$ & $>603.52$ & 70.49 & - \\
\hline TF19 & 317955 & 241029 & 4370721 & $>600.03$ & $>600.03$ & $>600.75$ & 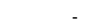 & $>600.03$ & $>610.63$ & 179.21 & - \\
\hline tomographic1 & 59360 & 45908 & 647495 & 119.00 & 33.46 & $>600.93$ & $>600.18$ & $>600.10$ & 11.05 & 22.58 & 1.61 \\
\hline Trec14 & 15904 & 3159 & 2872265 & 7.24 & 5.53 & 32.63 & $>58.91$ & 44.57 & 32.66 & 203.92 & 19.13 \\
\hline wheel_601 & 902103 & 723605 & 2170814 & $>600.05$ & $>600.06$ & $>600.77$ & $>600.07$ & $>600.40$ & $>631.06$ & $>600.76$ & \\
\hline
\end{tabular}

ACM Transactions on Mathematical Software, Vol. V, No. N, Article XXXX, Publication date: XXXX 2015. 
Table VI: Residuals Obtained for Subset CUTEst Problems by Each Method.

\begin{tabular}{|c|c|c|c|c|c|c|c|c|c|c|}
\hline name & no & diagonal & MIQR & RIF & BA-G & MI35 & MI30-MIN & MA87 & MA97 & SPQR \\
\hline BAS1LP & $5.54 \mathrm{E}+1$ & $5.54 \mathrm{E}+1$ & $5.54 \mathrm{E}+1$ & - & $5.54 \mathrm{E}+1$ & $5.54 \mathrm{E}+1$ & $5.54 \mathrm{E}+1$ & $5.54 \mathrm{E}+1$ & $5.54 \mathrm{E}+1$ & $5.54 \mathrm{E}+1$ \\
\hline BAXTER & $9.76 \mathrm{E}+1$ & & $9.18 \mathrm{E}+1$ & - & 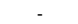 & - & & $5.93 \mathrm{E}+1$ & $6.50 \mathrm{E}+1$ & $5.92 \mathrm{E}+1$ \\
\hline BCDOUT & $3.54 \mathrm{E}+1$ & $3.53 \mathrm{E}+1$ & 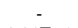 & 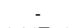 & 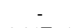 & $3.53 \mathrm{E}+1$ & $3.54 \mathrm{E}+1$ & $3.53 \mathrm{E}+1$ & $3.53 \mathrm{E}+1$ & $3.53 \mathrm{E}+1$ \\
\hline $\mathrm{COg}$ & $8.91 \mathrm{E}+1$ & $8.91 \mathrm{E}+1$ & $8.91 \mathrm{E}+1$ & $8.91 \mathrm{E}+1$ & $8.91 \mathrm{E}+1$ & $8.91 \mathrm{E}+1$ & $8.92 \mathrm{E}+1$ & $8.91 \mathrm{E}+1$ & $8.91 \mathrm{E}+1$ & $8.91 \mathrm{E}+1$ \\
\hline CONT11_L & $8.09 \mathrm{E}+2$ & $8.09 \mathrm{E}+2$ & - & $8.09 \mathrm{E}+2$ & $8.09 \mathrm{E}+2$ & $8.09 \mathrm{E}+2$ & $8.09 \mathrm{E}+2$ & $8.09 \mathrm{E}+2$ & $8.09 \mathrm{E}+2$ & $8.09 \mathrm{E}+2$ \\
\hline DBIR1 & $1.67 \mathrm{E}+2$ & $1.66 \mathrm{E}+2$ & $1.66 \mathrm{E}+2$ & 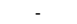 & $1.66 \mathrm{E}+2$ & $1.67 \mathrm{E}+2$ & - & $1.66 \mathrm{E}+2$ & $1.66 \mathrm{E}+2$ & $1.66 \mathrm{E}+2$ \\
\hline DBIR2 & $1.67 \mathrm{E}+2$ & $1.66 \mathrm{E}+2$ & $1.66 \mathrm{E}+2$ & & $1.66 \mathrm{E}+2$ & $1.67 \mathrm{E}+2$ & & $1.66 \mathrm{E}+2$ & $1.66 \mathrm{E}+2$ & $1.66 \mathrm{E}+2$ \\
\hline D2Q06C & $3.49 \mathrm{E}+1$ & $3.49 \mathrm{E}+1$ & $3.49 \mathrm{E}+1$ & $3.49 \mathrm{E}+1$ & $3.49 \mathrm{E}+1$ & $3.49 \mathrm{E}+1$ & $3.49 \mathrm{E}+1$ & $3.49 \mathrm{E}+1$ & $3.49 \mathrm{E}+1$ & $3.49 \mathrm{E}+1$ \\
\hline DELFO00 & $5.38 \mathrm{E}+1$ & $5.38 \mathrm{E}+1$ & $5.38 \mathrm{E}+1$ & $5.38 \mathrm{E}+1$ & $5.38 \mathrm{E}+1$ & $5.38 \mathrm{E}+1$ & $5.38 \mathrm{E}+1$ & $5.38 \mathrm{E}+1$ & $5.38 \mathrm{E}+1$ & $5.38 \mathrm{E}+1$ \\
\hline GE & $7.25 \mathrm{E}+1$ & $7.24 \mathrm{E}+1$ & $7.24 \mathrm{E}+1$ & $7.24 \mathrm{E}+1$ & $7.24 \mathrm{E}+1$ & $7.24 \mathrm{E}+1$ & $7.24 \mathrm{E}+1$ & $7.24 \mathrm{E}+1$ & $7.24 \mathrm{E}+1$ & $7.24 \mathrm{E}+1$ \\
\hline LARGE001 & $6.06 \mathrm{E}+1$ & $6.06 \mathrm{E}+1$ & $6.06 \mathrm{E}+1$ & $6.06 \mathrm{E}+1$ & $6.06 \mathrm{E}+1$ & $6.06 \mathrm{E}+1$ & $6.07 \mathrm{E}+1$ & $6.06 \mathrm{E}+1$ & $6.06 \mathrm{E}+1$ & $6.06 \mathrm{E}+1$ \\
\hline LPL1 & $7.08 \mathrm{E}+1$ & $7.08 \mathrm{E}+1$ & $7.08 \mathrm{E}+1$ & & $7.08 \mathrm{E}+1$ & $7.08 \mathrm{E}+1$ & $7.09 \mathrm{E}+1$ & $7.08 \mathrm{E}+1$ & $7.08 \mathrm{E}+1$ & $7.08 \mathrm{E}+1$ \\
\hline MOD2 & $1.38 \mathrm{E}+2$ & $1.38 \mathrm{E}+2$ & $1.38 \mathrm{E}+2$ & $1.38 \mathrm{E}+2$ & $1.38 \mathrm{E}+2$ & $1.38 \mathrm{E}+2$ & $1.39 \mathrm{E}+2$ & $1.38 \mathrm{E}+2$ & $1.38 \mathrm{E}+2$ & $1.38 \mathrm{E}+2$ \\
\hline MODEL10 & $5.35 \mathrm{E}+1$ & $5.35 \mathrm{E}+1$ & $5.35 \mathrm{E}+1$ & $5.35 \mathrm{E}+1$ & $5.35 \mathrm{E}+1$ & $5.35 \mathrm{E}+1$ & $5.36 \mathrm{E}+1$ & $5.35 \mathrm{E}+1$ & $5.35 \mathrm{E}+1$ & $5.35 \mathrm{E}+1$ \\
\hline MPSBCD 03 & $3.53 \mathrm{E}+1$ & $3.53 \mathrm{E}+1$ & - & 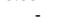 & - & $3.53 \mathrm{E}+1$ & $3.53 \mathrm{E}+1$ & $3.52 \mathrm{E}+1$ & $3.52 \mathrm{E}+1$ & $3.52 \mathrm{E}+1$ \\
\hline $\begin{array}{l}\text { NSCT2 } \\
\text { NST }\end{array}$ & $1.83 \mathrm{E}+2$ & $1.83 \mathrm{E}+2$ & $1.83 \mathrm{E}+2$ & - & $1.83 \mathrm{E}+2$ & $1.83 \mathrm{E}+2$ & & $1.83 \mathrm{E}+2$ & $1.83 \mathrm{E}+2$ & $1.83 \mathrm{E}+2$ \\
\hline NSIR2 & $8.05 \mathrm{E}+1$ & $8.04 \mathrm{E}+1$ & $8.04 \mathrm{E}+1$ & - & $8.04 \mathrm{E}+1$ & $8.05 \mathrm{E}+1$ & $8.05 \mathrm{E}+1$ & $8.04 \mathrm{E}+1$ & $8.04 \mathrm{E}+1$ & $8.04 \mathrm{E}+1$ \\
\hline PDE1 & $3.03 \mathrm{E}+2$ & $3.03 \mathrm{E}+2$ & & & & & & & $3.03 \mathrm{E}+2$ & \\
\hline PDS- 100 & $2.84 \mathrm{E}+2$ & $2.84 \mathrm{E}+2$ & $2.84 \mathrm{E}+2$ & $2.84 \mathrm{E}+2$ & $2.84 \mathrm{E}+2$ & $2.84 \mathrm{E}+2$ & $2.85 \mathrm{E}+2$ & $2.84 \mathrm{E}+2$ & $2.84 \mathrm{E}+2$ & $2.84 \mathrm{E}+2$ \\
\hline PDS -90 & $2.68 \mathrm{E}+2$ & $2.68 \mathrm{E}+2$ & $2.68 \mathrm{E}+2$ & $2.68 \mathrm{E}+2$ & $2.68 \mathrm{E}+2$ & $2.68 \mathrm{E}+2$ & $2.68 \mathrm{E}+2$ & $2.68 \mathrm{E}+2$ & $2.68 \mathrm{E}+2$ & $2.68 \mathrm{E}+2$ \\
\hline PILOT-JA & $3.32 \mathrm{E}+1$ & $3.19 \mathrm{E}+1$ & $3.19 \mathrm{E}+1$ & $3.19 \mathrm{E}+1$ & $3.19 \mathrm{E}+1$ & $3.19 \mathrm{E}+1$ & $3.20 \mathrm{E}+1$ & $3.19 \mathrm{E}+1$ & $3.19 \mathrm{E}+1$ & $3.19 \mathrm{E}+1$ \\
\hline PILOTNOV & $3.50 \mathrm{E}+1$ & $3.28 \mathrm{E}+1$ & $3.28 \mathrm{E}+1$ & $3.28 \mathrm{E}+1$ & $3.28 \mathrm{E}+1$ & $3.28 \mathrm{E}+1$ & $3.29 \mathrm{E}+1$ & $3.28 \mathrm{E}+1$ & $3.28 \mathrm{E}+1$ & $3.28 \mathrm{E}+1$ \\
\hline RAIL2586 & $1.41 \mathrm{E}+2$ & $1.41 \mathrm{E}+2$ & $1.41 \mathrm{E}+2$ & $1.41 \mathrm{E}+2$ & $1.41 \mathrm{E}+2$ & $1.41 \mathrm{E}+2$ & $1.41 \mathrm{E}+2$ & $1.41 \mathrm{E}+2$ & $1.41 \mathrm{E}+2$ & $1.41 \mathrm{E}+2$ \\
\hline RAIL4284 & $1.69 \mathrm{E}+2$ & $1.69 \mathrm{E}+2$ & $1.69 \mathrm{E}+2$ & $1.69 \mathrm{E}+2$ & $1.69 \mathrm{E}+2$ & $1.69 \mathrm{E}+2$ & $1.69 \mathrm{E}+2$ & $1.69 \mathrm{E}+2$ & $1.69 \mathrm{E}+2$ & - \\
\hline SPAL_004 & - & $5.65 \mathrm{E}-6$ & & & $3.24 \mathrm{E}-11$ & $5.65 \mathrm{E}-6$ & 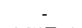 & $5.31 \mathrm{E}-8$ & -2 & . \\
\hline STAT96V2 & $9.72 \mathrm{E}+2$ & $9.72 \mathrm{E}+2$ & $9.72 \mathrm{E}+2$ & $9.72 \mathrm{E}+2$ & $9.72 \mathrm{E}+2$ & $9.72 \mathrm{E}+2$ & $9.73 \mathrm{E}+2$ & $9.72 \mathrm{E}+2$ & $9.72 \mathrm{E}+2$ & $9.72 \mathrm{E}+2$ \\
\hline STAT96V3 & $1.04 \mathrm{E}+3$ & $1.04 \mathrm{E}+3$ & $1.04 \mathrm{E}+3$ & $1.04 \mathrm{E}+3$ & $1.04 \mathrm{E}+3$ & $1.04 \mathrm{E}+3$ & $1.05 \mathrm{E}+3$ & $1.04 \mathrm{E}+3$ & $1.04 \mathrm{E}+3$ & $1.04 \mathrm{E}+3$ \\
\hline STAT96V4 & $1.20 \mathrm{E}+2$ & $1.20 \mathrm{E}+2$ & $1.20 \mathrm{E}+2$ & $1.20 \mathrm{E}+2$ & $1.20 \mathrm{E}+2$ & $1.20 \mathrm{E}+2$ & $1.21 \mathrm{E}+2$ & $1.20 \mathrm{E}+2$ & $1.20 \mathrm{E}+2$ & $1.20 \mathrm{E}+2$ \\
\hline STORMG $21 \mathrm{~K}$ & $8.96 \mathrm{E}+2$ & $8.96 \mathrm{E}+2$ & & & & $8.96 \mathrm{E}+2$ & & $8.96 \mathrm{E}+2$ & & \\
\hline WATSON_1 & $2.54 \mathrm{E}+2$ & $2.54 \mathrm{E}+2$ & $2.54 \mathrm{E}+2$ & $2.54 \mathrm{E}+2$ & $2.54 \mathrm{E}+2$ & $2.54 \mathrm{E}+2$ & $2.55 \mathrm{E}+2$ & $2.54 \mathrm{E}+2$ & $2.54 \mathrm{E}+2$ & $2.54 \mathrm{E}+2$ \\
\hline WATSON_2 & $3.35 \mathrm{E}+2$ & $3.35 \mathrm{E}+2$ & $3.35 \mathrm{E}+2$ & $3.35 \mathrm{E}+2$ & $3.35 \mathrm{E}+2$ & $3.35 \mathrm{E}+2$ & $3.36 \mathrm{E}+2$ & $3.35 \mathrm{E}+2$ & $3.35 \mathrm{E}+2$ & $3.35 \mathrm{E}+2$ \\
\hline WORLD & $1.40 \mathrm{E}+2$ & $1.40 \mathrm{E}+2$ & $1.40 \mathrm{E}+2$ & $1.40 \mathrm{E}+2$ & $1.40 \mathrm{E}+2$ & $1.40 \mathrm{E}+2$ & $1.41 \mathrm{E}+2$ & $1.40 \mathrm{E}+2$ & $1.40 \mathrm{E}+2$ & $1.40 \mathrm{E}+2$ \\
\hline
\end{tabular}


Table VI: Residuals Obtained for Subset UF Problems by Each Method.

\begin{tabular}{|c|c|c|c|c|c|c|c|c|c|c|}
\hline name & no & diagonal & MIQR & RIF & BA-G & MI35 & MI30-MIN & MA87 & MA97 & SPQR \\
\hline 12month1 & & $9.27 \mathrm{E}+2$ & $9.27 \mathrm{E}+2$ & & $9.27 \mathrm{E}+2$ & $9.27 \mathrm{E}+2$ & $9.27 \mathrm{E}+2$ & $9.27 \mathrm{E}+2$ & & \\
\hline 162bit & $1.17 \mathrm{E}+1$ & $1.17 \mathrm{E}+1$ & $1.17 \mathrm{E}+1$ & $1.17 \mathrm{E}+1$ & $1.17 \mathrm{E}+1$ & $1.17 \mathrm{E}+1$ & $1.18 \mathrm{E}+1$ & $1.17 \mathrm{E}+1$ & $1.17 \mathrm{E}+1$ & $1.17 \mathrm{E}+1$ \\
\hline 176bit & $1.84 \mathrm{E}+1$ & $1.84 \mathrm{E}+1$ & $1.84 \mathrm{E}+1$ & $1.84 \mathrm{E}+1$ & $1.84 \mathrm{E}+1$ & $1.84 \mathrm{E}+1$ & $1.84 \mathrm{E}+1$ & $1.84 \mathrm{E}+1$ & $1.84 \mathrm{E}+1$ & $1.84 \mathrm{E}+1$ \\
\hline $192 \mathrm{bit}$ & $2.48 \mathrm{E}+1$ & $2.48 \mathrm{E}+1$ & $2.48 \mathrm{E}+1$ & $2.48 \mathrm{E}+1$ & $2.48 \mathrm{E}+1$ & $2.48 \mathrm{E}+1$ & $2.49 \mathrm{E}+1$ & $2.48 \mathrm{E}+1$ & $2.48 \mathrm{E}+1$ & $2.48 \mathrm{E}+1$ \\
\hline 208bit & $3.86 \mathrm{E}+1$ & $3.85 \mathrm{E}+1$ & $3.84 \mathrm{E}+1$ & $3.85 \mathrm{E}+1$ & $3.84 \mathrm{E}+1$ & $3.84 \mathrm{E}+1$ & $3.85 \mathrm{E}+1$ & $3.84 \mathrm{E}+1$ & $3.84 \mathrm{E}+1$ & $3.84 \mathrm{E}+1$ \\
\hline beaflw & $5.05 \mathrm{E}+0$ & $4.57 \mathrm{E}+0$ & $4.60 \mathrm{E}+0$ & & $4.33 \mathrm{E}+0$ & $4.68 \mathrm{E}+0$ & - & $4.37 \mathrm{E}+0$ & $4.52 \mathrm{E}+0$ & $4.16 \mathrm{E}+0$ \\
\hline c8_mat11 & $2.21 \mathrm{E}+1$ & $2.19 \mathrm{E}+1$ & & & & $2.19 \mathrm{E}+1$ & & $2.12 \mathrm{E}+1$ & $2.14 \mathrm{E}+1$ & $2.12 \mathrm{E}+1$ \\
\hline connectus & $6.27 \mathrm{E}+2$ & $6.27 \mathrm{E}+2$ & $6.27 \mathrm{E}+2$ & $6.27 \mathrm{E}+2$ & $6.27 \mathrm{E}+2$ & $6.27 \mathrm{E}+2$ & $6.28 \mathrm{E}+2$ & $6.27 \mathrm{E}+2$ & $6.27 \mathrm{E}+2$ & $6.27 \mathrm{E}+2$ \\
\hline ESOC & $4.04 \mathrm{E}+2$ & $2.23 \mathrm{E}+1$ & - & - & - & 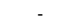 & - & $3.64 \mathrm{E}-1$ & $1.51 \mathrm{E}+1$ & $5.97 \mathrm{E}-9$ \\
\hline EternityIIEtilde & $4.47 \mathrm{E}-6$ & $4.43 \mathrm{E}-6$ & $4.37 \mathrm{E}-6$ & $4.47 \mathrm{E}-6$ & $5.33 \mathrm{E}-6$ & $4.33 \mathrm{E}-6$ & $1.19 \mathrm{E}-4$ & $1.03 \mathrm{E}-7$ & $1.03 \mathrm{E}-5$ & $1.08 \mathrm{E}-12$ \\
\hline f855_mat9 & $1.79 \mathrm{E}+1$ & $1.79 \mathrm{E}+1$ & $1.75 \mathrm{E}+1$ & $1.67 \mathrm{E}+1$ & - & $1.79 \mathrm{E}+1$ & - & $1.34 \mathrm{E}+1$ & $1.60 \mathrm{E}+1$ & $1.90 \mathrm{E}+4$ \\
\hline GL7d16 & $7.48 \mathrm{E}+2$ & $7.48 \mathrm{E}+2$ & $7.48 \mathrm{E}+2$ & 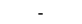 & $7.48 \mathrm{E}+2$ & $7.48 \mathrm{E}+2$ & $7.49 \mathrm{E}+2$ & - & - & \\
\hline GL7d17 & $8.98 \mathrm{E}+2$ & $8.98 \mathrm{E}+2$ & - & - & $8.98 \mathrm{E}+2$ & $8.98 \mathrm{E}+2$ & - & - & - & - \\
\hline GL7d18 & $9.31 \mathrm{E}+2$ & $9.31 \mathrm{E}+2$ & - & - & $9.31 \mathrm{E}+2$ & $9.31 \mathrm{E}+2$ & - & - & - & - \\
\hline GL7d19 & $1.04 \mathrm{E}+3$ & $1.04 \mathrm{E}+3$ & - & - & $1.04 \mathrm{E}+3$ & $1.04 \mathrm{E}+3$ & - & - & - & 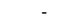 \\
\hline GL7d20 & $1.09 \mathrm{E}+3$ & $1.09 \mathrm{E}+3$ & - & - & $1.09 \mathrm{E}+3$ & $1.09 \mathrm{E}+3$ & - & - & - & - \\
\hline GL $7 \mathrm{~d} 21$ & $1.00 \mathrm{E}+3$ & $1.00 \mathrm{E}+3$ & - & - & $1.00 \mathrm{E}+3$ & $1.00 \mathrm{E}+3$ & & - & - & - \\
\hline GL7d22 & $7.89 \mathrm{E}+2$ & $7.89 \mathrm{E}+2$ & $7.89 \mathrm{E}+2$ & - & $7.89 \mathrm{E}+2$ & $7.89 \mathrm{E}+2$ & $7.89 \mathrm{E}+2$ & - & - & - \\
\hline GL7d23 & $5.35 \mathrm{E}+2$ & $5.35 \mathrm{E}+2$ & $5.35 \mathrm{E}+2$ & & $5.35 \mathrm{E}+2$ & $5.35 \mathrm{E}+2$ & $5.36 \mathrm{E}+2$ & - & & \\
\hline graphics & - & $3.03 \mathrm{E}-4$ & $3.03 \mathrm{E}-4$ & $3.03 \mathrm{E}-4$ & - & $3.03 \mathrm{E}-4$ & $3.03 \mathrm{E}-4$ & $3.55 \mathrm{E}+0$ & $3.93 \mathrm{E}+1$ & $3.03 \mathrm{E}-4$ \\
\hline HFE18_96_in & $4.91 \mathrm{E}-1$ & $4.90 \mathrm{E}-1$ & $4.90 \mathrm{E}-1$ & $4.90 \mathrm{E}-1$ & $4.90 \mathrm{E}-1$ & $4.90 \mathrm{E}-1$ & $4.91 \mathrm{E}-1$ & $4.90 \mathrm{E}-1$ & $4.90 \mathrm{E}-1$ & $4.90 \mathrm{E}-1$ \\
\hline IG5-15 & $5.48 \mathrm{E}+1$ & $5.48 \mathrm{E}+1$ & $5.48 \mathrm{E}+1$ & $5.48 \mathrm{E}+1$ & $5.48 \mathrm{E}+1$ & $5.48 \mathrm{E}+1$ & $5.49 \mathrm{E}+1$ & $5.48 \mathrm{E}+1$ & $5.48 \mathrm{E}+1$ & $5.48 \mathrm{E}+1$ \\
\hline IG5-16 & $7.15 \mathrm{E}+1$ & $7.15 \mathrm{E}+1$ & $7.15 \mathrm{E}+1$ & $7.15 \mathrm{E}+1$ & $7.15 \mathrm{E}+1$ & $7.15 \mathrm{E}+1$ & $7.15 \mathrm{E}+1$ & $7.15 \mathrm{E}+1$ & $7.15 \mathrm{E}+1$ & $7.15 \mathrm{E}+1$ \\
\hline IG5-17 & $9.10 \mathrm{E}+1$ & $9.10 \mathrm{E}+1$ & $9.10 \mathrm{E}+1$ & $9.10 \mathrm{E}+1$ & $9.10 \mathrm{E}+1$ & $9.10 \mathrm{E}+1$ & $9.10 \mathrm{E}+1$ & $9.10 \mathrm{E}+1$ & $9.10 \mathrm{E}+1$ & $9.10 \mathrm{E}+1$ \\
\hline IG5-18 & $1.15 \mathrm{E}+2$ & $1.15 \mathrm{E}+2$ & $1.15 \mathrm{E}+2$ & $1.15 \mathrm{E}+2$ & $1.15 \mathrm{E}+2$ & $1.15 \mathrm{E}+2$ & $1.15 \mathrm{E}+2$ & $1.15 \mathrm{E}+2$ & $1.15 \mathrm{E}+2$ & $1.15 \mathrm{E}+2$ \\
\hline IMDB & & & - & - & - & & & & 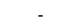 & \\
\hline kneser_10_4_1 & $1.62 \mathrm{E}+2$ & $1.62 \mathrm{E}+2$ & - & & - & $1.62 \mathrm{E}+2$ & $1.62 \mathrm{E}+2$ & $1.62 \mathrm{E}+2$ & - & $1.63 \mathrm{E}+2$ \\
\hline landmark & $1.31 \mathrm{E}-5$ & $1.12 \mathrm{E}-5$ & $1.12 \mathrm{E}-5$ & $1.12 \mathrm{E}-5$ & $1.15 \mathrm{E}-5$ & $1.12 \mathrm{E}-5$ & $8.59 \mathrm{E}-5$ & $1.12 \mathrm{E}-5$ & $1.12 \mathrm{E}-5$ & $1.12 \mathrm{E}-5$ \\
\hline LargeRegFile & $4.44 \mathrm{E}+2$ & $4.44 \mathrm{E}+2$ & $4.44 \mathrm{E}+2$ & - & $4.44 \mathrm{E}+2$ & $4.44 \mathrm{E}+2$ & $4.44 \mathrm{E}+2$ & $4.44 \mathrm{E}+2$ & $4.44 \mathrm{E}+2$ & $4.44 \mathrm{E}+2$ \\
\hline Maragal_6 & $9.39 \mathrm{E}+1$ & $9.39 \mathrm{E}+1$ & $9.38 \mathrm{E}+1$ & - & $9.38 \mathrm{E}+1$ & $9.39 \mathrm{E}+1$ & $9.39 \mathrm{E}+1$ & $9.38 \mathrm{E}+1$ & $9.38 \mathrm{E}+1$ & $9.48 \mathrm{E}+1$ \\
\hline Maragal_7 & $1.33 \mathrm{E}+2$ & $1.33 \mathrm{E}+2$ & $1.33 \mathrm{E}+2$ & - & $1.33 \mathrm{E}+2$ & $1.33 \mathrm{E}+2$ & $1.33 \mathrm{E}+2$ & $1.33 \mathrm{E}+2$ & $1.33 \mathrm{E}+2$ & $1.34 \mathrm{E}+2$ \\
\hline Maragal_8 & - & - & - & - & - & - & - & $2.38 \mathrm{E}+2$ & $2.38 \mathrm{E}+2$ & $2.37 \mathrm{E}+2$ \\
\hline mri1 & $2.67 \mathrm{E}+1$ & $2.67 \mathrm{E}+1$ & $2.67 \mathrm{E}+1$ & $2.67 \mathrm{E}+1$ & $2.67 \mathrm{E}+1$ & $2.67 \mathrm{E}+1$ & $2.67 \mathrm{E}+1$ & $2.67 \mathrm{E}+1$ & $2.67 \mathrm{E}+1$ & $3.56 \mathrm{E}+13$ \\
\hline $\begin{array}{l}\text { mri2 } \\
\text { NotreDame_actors }\end{array}$ & $1.41 \mathrm{E}+2$ & $1.41 \mathrm{E}+2$ & $1.41 \mathrm{E}+2$ & 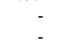 & $1.41 \mathrm{E}+2$ & $1.41 \mathrm{E}+2$ & $1.41 \mathrm{E}+2$ & $\begin{array}{l}1.41 \mathrm{E}+2 \\
5.18 \mathrm{E}+2\end{array}$ & - & $1.25 \mathrm{E}+24$ \\
\hline psse0 & $1.62 \mathrm{E}+2$ & $1.62 \mathrm{E}+2$ & $1.62 \mathrm{E}+2$ & $1.62 \mathrm{E}+2$ & $1.62 \mathrm{E}+2$ & $1.62 \mathrm{E}+2$ & $1.63 \mathrm{E}+2$ & $1.62 \mathrm{E}+2$ & $1.62 \mathrm{E}+2$ & 1. $62 \mathrm{E}+2$ \\
\hline psse 1 & $5.44 \mathrm{E}+1$ & $5.43 \mathrm{E}+1$ & $5.43 \mathrm{E}+1$ & - & $5.43 \mathrm{E}+1$ & $5.43 \mathrm{E}+1$ & $5.44 \mathrm{E}+1$ & $5.43 \mathrm{E}+1$ & $5.43 \mathrm{E}+1$ & $5.43 \mathrm{E}+1$ \\
\hline psse2 & $1.65 \mathrm{E}+2$ & $1.65 \mathrm{E}+2$ & $1.65 \mathrm{E}+2$ & & & $1.65 \mathrm{E}+2$ & $1.66 \mathrm{E}+2$ & $1.65 \mathrm{E}+2$ & $1.65 \mathrm{E}+2$ & $1.65 \mathrm{E}+2$ \\
\hline rel9 & $1.54 \mathrm{E}+3$ & $1.54 \mathrm{E}+3$ & $1.54 \mathrm{E}+3$ & - & $1.54 \mathrm{E}+3$ & $1.54 \mathrm{E}+3$ & - & - & 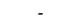 & 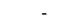 \\
\hline relat9 & $3.05 \mathrm{E}+3$ & $3.05 \mathrm{E}+3$ & $3.05 \mathrm{E}+3$ & & $3.05 \mathrm{E}+3$ & $3.05 \mathrm{E}+3$ & & - & & \\
\hline Rucci1 & . & $7.27 \mathrm{E}+2$ & - & $7.27 \mathrm{E}+2$ & 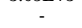 & - & $7.28 \mathrm{E}+2$ & $7.27 \mathrm{E}+2$ & $7.27 \mathrm{E}+2$ & $7.27 \mathrm{E}+2$ \\
\hline sls & $1.29 \mathrm{E}-5$ & $1.23 \mathrm{E}-5$ & $1.28 \mathrm{E}-5$ & $1.18 \mathrm{E}-5$ & $2.09 \mathrm{E}-4$ & $1.01 \mathrm{E}-5$ & $1.73 \mathrm{E}-5$ & $1.06 \mathrm{E}-7$ & $1.06 \mathrm{E}-5$ & - \\
\hline TF14 & $5.57 \mathrm{E}-7$ & $5.61 \mathrm{E}-7$ & $5.50 \mathrm{E}-7$ & $5.41 \mathrm{E}-7$ & $5.37 \mathrm{E}-9$ & $5.61 \mathrm{E}-7$ & $6.23 \mathrm{E}-3$ & $6.34 \mathrm{E}-8$ & $6.29 \mathrm{E}-6$ & $5.63 \mathrm{E}-14$ \\
\hline TF15 & $8.78 \mathrm{E}-7$ & $8.77 \mathrm{E}-7$ & $8.79 \mathrm{E}-7$ & $8.69 \mathrm{E}-7$ & - & $8.77 \mathrm{E}-7$ & $1.13 \mathrm{E}-2$ & $2.40 \mathrm{E}-7$ & $2.28 \mathrm{E}-5$ & $1.35 \mathrm{E}-13$ \\
\hline TF16 & $1.38 \mathrm{E}-6$ & $1.38 \mathrm{E}-6$ & - & $1.38 \mathrm{E}-6$ & - & $1.38 \mathrm{E}-6$ & $1.52 \mathrm{E}-2$ & $9.42 \mathrm{E}-7$ & $6.96 \mathrm{E}-5$ & $3.16 \mathrm{E}-13$ \\
\hline TF17 & - & 2000 & - & . & - & - & $2.14 \mathrm{E}-2$ & $3.66 \mathrm{E}-6$ & - & - \\
\hline TF18 & - & - & - & - & - & - & $3.19 \mathrm{E}-2$ & - & - & - \\
\hline TF19 & - & . & - & - & - & - & $4.26 \mathrm{E}-2$ & - & - & . \\
\hline tomographic1 & $4.20 \mathrm{E}+1$ & $4.19 \mathrm{E}+1$ & - & - & - & $4.19 \mathrm{E}+1$ & $4.19 \mathrm{E}+1$ & $4.18 \mathrm{E}+1$ & $4.18 \mathrm{E}+1$ & $4.18 \mathrm{E}+1$ \\
\hline Trec14 & $1.12 \mathrm{E}+2$ & $1.12 \mathrm{E}+2$ & $1.12 \mathrm{E}+2$ & - & $1.12 \mathrm{E}+2$ & $1.12 \mathrm{E}+2$ & $1.12 \mathrm{E}+2$ & $1.12 \mathrm{E}+2$ & $1.12 \mathrm{E}+2$ & $1.12 \mathrm{E}+2$ \\
\hline wheel_601 & 2010 & 2010 & thes & - & 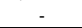 & - & 年 & 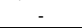 & $4.22 \mathrm{E}+2$ & (1) \\
\hline
\end{tabular}

ACM Transactions on Mathematical Software, Vol. V, No. N, Article XXXX, Publication date: XXXX 2015. 